\title{
EMBEDDING AND KNOTTING OF MANIFOLDS IN EUCLIDEAN SPACES
}

\author{
ARKADIY SKOPENKOV
}

\begin{abstract}
A clear understanding of topology of higher-dimensional objects is important in many branches of both pure and applied mathematics. In this survey we attempt to present some results of higher-dimensional topology in a way which makes clear the visual and intuitive part of the constructions and the arguments. In particular, we show how abstract algebraic constructions appear naturally in the study of geometric problems. Before giving a general construction, we illustrate the main ideas in simple but important particular cases, in which the essence is not veiled by technicalities.

More specifically, we present several classical and modern results on the embedding and knotting of manifolds in Euclidean space. We state many concrete results (in particular, recent explicit classification of knotted tori). Their statements (but not proofs!) are simple and accessible to non-specialists. We outline a general approach to embeddings via the classical van Kampen-Shapiro-Wu-Haefliger-Weber 'deleted product' obstruction. This approach reduces the isotopy classification of embeddings to the homotopy classification of equivariant maps, and so implies the above concrete results. We describe the revival of interest in this beautiful branch of topology, by presenting new results in this area (of Freedman, Krushkal, Teichner, Segal, Spież and the author): a generalization the Haefliger-Weber embedding theorem below the metastable dimension range and examples showing that other analogues of this theorem are false outside the metastable dimension range.
\end{abstract}

\section{Introduction}

2. The simplest-to-state results on embeddings

3. Links and knotted tori

4. The van Kampen obstruction

5. The Haefliger-Wu invariant

6. On the deleted product of the torus

7. Borromean rings and the Haefliger-Wu invariant

8. The disjunction method

References

1991 Mathematics Subject Classification. Primary: 57-02, 57R40, 57Q35; Secondary: 55S15, 35, 91, 57Q30, 60, 65, 57M15, 57N30, 35, 37, 40, 45, 50, 75, 57Q30, 37, 40, 45, 65, 57R20, 52.

Key words and phrases. Embedding, isotopy, deleted product, self-intersection set, metastable case, knotted tori, equivariant maps, general position, characteristic classes, the van Kampen obstruction.

This paper is to be published in London Mathematical Society Lecture Notes, Surveys in geometry and number theory: Reports on contemporary Russian mathematics. The author gratefully acknowledges the support of a grant from the London Mathematical Society via the programme 'Invitation of young Russian mathematicians', by INTAS Grant No. YSF-2002-393, by the Russian Foundation for Basic Research, Grants No 05-01-00993 and 04-01-00682, President of Russian Federation Grants MD-3938.2005.1 and NSH-1988.2003.1, and by the Pierre Deligne fund based on his 2004 Balzan prize in mathematics.

Typeset by $\mathcal{A} \mathcal{M} \mathcal{S}-\mathrm{T}_{\mathrm{E}} \mathrm{X}$ 


\section{INTRODUCTION}

Embedding and Knotting Problems. Many theorems in mathematics state that an arbitrary object of a given abstractly defined class is a subobject of a certain 'standard' object of this class. Such are the Cayley theorem on the embedding of finite groups into the symmetric groups, the theorem on the representation of compact Lie groups as subgroups of $G L(V)$ for a certain linear space $V$, the Urysohn theorem on the embedding of normal spaces with countable basis into the Hilbert space, the general position theorem on the embedding of finite polyhedra into $\mathbb{R}^{m}$, the Menger-Nöbeling-Pontryagin theorem on the embedding of finite-dimensional compact spaces into $\mathbb{R}^{m}$, the Whitney theorem on the embedding of smooth manifolds into $\mathbb{R}^{m}$, the Nash theorem on the embedding of Riemannian manifolds into $\mathbb{R}^{m}$, the Gromov theorem on the embedding of symplectic manifolds into $\mathbb{R}^{2 n}$, etc. The solution of the 13 th Hilbert problem by Kolmogorov and Arnold can also be formulated in terms of embeddings. Although interesting in themselves, these embeddability theorems also prove to be powerful tools for solving other problems. Subtler problem about the embeddability of a given space into $\mathbb{R}^{m}$ for a given $m$, as well as about counting such embeddings, are among the most important classical problems of topology.

According to Zeeman, the classical problems of topology are the following.

1) The Homeomorphism Problem: When are two given spaces homeomorphic?

2) The Embedding Problem: When does a given space embed into $\mathbb{R}^{m}$ ?

3) The Knotting Problem: When are two given embeddings isotopic?

Definitions of an embedding and an isotopy are recalled in the next subsection.

Embedding and Knotting Problems have played an outstanding role in the development of topology. Various methods for the investigation of these problems were created by such classical figures as G. Alexander, H. Hopf, E. van Kampen, K. Kuratowski, S. MacLane, L.S. Pontryagin, R. Thom, H. Whitney, W. Browder, A. Haefliger, M. Hirsch, J. F. P. Hudson, M. Irwin, J. Levine, S. Novikov, R. Penrose, J. H. C. Whitehead, C. Zeeman and others. For surveys see [Wu65, Introduction, Gi71, Ad93, RS96, RS99]. Nowadays interest in this subject is reviving.

There are only a few cases in which a concrete answer to the Embedding and Knotting Problems can be given. E. g. for the best known specific case of the Knotting Problem, i.e. for the theory of codimension 2 embeddings (in particular, for the classical theory of knots in $\mathbb{R}^{3}$ ), a complete concrete classification is neither known nor expected. A concrete complete description of a (non-empty) set of embeddings of a given manifold up to isotopy is only known either just below the stable range, or for highly-connected manifolds, or for links and knotted tori. (An concrete complete answer to the Embedding Problem was obtained additionally for projective spaces [Gi71], for products of low-dimensional manifolds or of graphs [ARS01, Sk03] and for certain twisted products [Re71, RS02].) Therefore Knotting Problem is one of the hardest problems in topology. The Embedding Problem is also hard for similar reasons. However, the statements (but not the proofs!) are simple and accessible to non-specialists. One of the purposes of this survey is to list such statements. They are presented in $\S 2$ and $\S 3$. Statements analogous to those presented (e.g. for non-closed manifolds) are often omitted.

Another purpose of this survey is to outline a general approach useful for obtaining such concrete complete results. There are interesting approaches giving nice theoretical results. The author is grateful to M. Weiss for indicating that the approach of [GW99, We] 
gives also concrete results on homotopy type of the space of embeddings $S^{1} \rightarrow \mathbb{R}^{n}$. The application of surgery [Le65, Br68, Ha62, Ha66, Ha66', Ha86, CRS04] gives good concrete results for simplest manifolds. The advantage of the surgery approach (comparatively to the deleted product approach, see below) is that it works in the presence of smooth knots $S^{n} \rightarrow \mathbb{R}^{m}$. The disadvantage is that it uses the homotopy type of the complement and description of possible normal bundles, and so faces computational difficulties even for relatively simple manifolds like tori, see $\S 3$ (for a successful attempt to overcome this problem see [KS05, Sk06]). According to Wall, surgery only reduces geometric problems on embeddings to algebraic problems which are even harder to solve [Wa70].

The method of the Haefliger-Wu invariant (or the deleted product method) gives many concrete results. We introduce this invariant in $\S 5$. In particular, most of the results from $\S 2, \S 3$ and $\S 4$ can be deduced by the deleted product method (although originally some of them were proved directly, sometimes in a weaker form). The deleted product method is a demonstration of a general mathematical idea of 'complements of diagonals' and the 'Gauss mapping' which appeared in works of Borsuk and Lefschetz around 1930. The Haefliger-Wu invariant generalizes the linking coefficient, the Whitney obstruction and the van Kampen obstruction. The deleted product method in the theory of embeddings was developed by van Kampen (1932), Shapiro (1957), Wu (1957-59), Haefliger (1962), Weber (1967), Harris (1969) and others. The Van-Kampen-Shaprio-Wu approach works for embeddings of polyhedra, but is closely related to embeddings of manifolds and so is presented in $\S 4$. The classical Haefliger-Weber Theorem 5.4 asserts the bijectivity of the Haefliger-Wu invariant for embeddings of $n$-dimensional polyhedra and manifolds into $\mathbb{R}^{m}$ under the 'metastable' dimension restriction

$$
2 m \geq 3 n+4 .
$$

Other embedding invariants (obtained using $p$-fold deleted products, see end of $\S 5$, or using complement together with normal bundle [Br68, CRS04]) are hard to compute. So the investigation of embeddings for $2 m<3 n+4$ naturally leads to the problem of finding conditions under which the Haefliger-Wu invariant is complete without the metastable dimension assumption. There were many examples showing that for embeddings of manifolds the metastable dimension restriction is sharp in many senses (Boechat, Freedman, Haefliger, Hsiang, Krushkal, Levine, Mardesic, Segal, Skopenkov, Spiez, Szarba, Teichner, Zeeman, see $\S 5$ ). So it is surprising (Theorem 5.5) that for $d$-connected $n$-dimensional manifolds and in the piecewise linear category the metastable restriction in the HaefligerWeber Theorem can be weakened to

$$
2 m \geq 3 n+3-d .
$$

We present many beautiful examples motivated by the Embedding and Isotopy Problems. In particular, in $\S 2$ we present a construction of the Hudson torus (which is simpler than the original one), in $\S 3$ we construct examples illustrating the distinction between piecewise linear and smooth embeddings. In $\S 6$ we prove some results on the deleted product of 'torus' $S^{p} \times S^{q}$ and on the Haefliger-Wu invariant of knotted tori. In $\S 6$ and $\S 7$ we construct most of the examples of the incompleteness of the van Kampen obstruction and the Haefliger-Wu invariant, announced in $\S 5$ and $\S 4$. The construction of these examples is based on knotted tori ( $\S 6)$ or on (higher-dimensional) Casson finger moves ( $\S 7)$. For some other examples we only give references. 
The Haefliger-Weber theorem and its analogue below the metastable case were obtained by a combination of and the improvement of methods and results from various parts of topology: the theory of immersions, homotopy theory, engulfing, the Whitney trick, van Kampen finger moves, the Freedman-Krushkal-Teichner trick and their generalizations. The most important method is the disjunction method (end of $\S 4$ and $\S 8$ ). These methods are also applied in other areas. In $\S 8$ we prove the surjectivity of the Haefliger-Wu invariant in the piecewise linear case. For the reader's convenience, we take a historical approach to the exposition: the disjunction method is applied in its complete generality only after illustration in simpler particular cases. We also prove the analogue of the Haefliger-Weber theorem below the metastable range for the simplest case. We do not prove many other results of $\S 2-\S 5$ but give references and sometimes sketch the proofs.

Sections $\S 6, \S 7$ and $\S 8$ depend on $\S 5$. Otherwise the sections are independent of each other except for minor details that can well be omitted during the first reading.

Definitions and notations. A smooth embedding is a smooth injective map $f: N \rightarrow \mathbb{R}^{m}$ such that $d f$ is a monomorphism at each point.

By a polyhedron we shall understand a compact polyhedron. A map $f: N \rightarrow \mathbb{R}^{m}$ of a polyhedron $N$ is piecewise-smooth if it is smooth on each simplex of some smooth triangulation of $N$. We denote the piecewise-smooth category by PL. This is the usual notation for the piecewise-linear category but the classification of piecewise-smooth embeddings (or immersions) coincides with the classification of piecewise linear embeddings (or immersions) [Ha67]. A PL embedding is a PL injective map $f: N \rightarrow \mathbb{R}^{m}$.

We write CAT for DIFF or PL. We often omit CAT if a statement holds in both PL and DIFF categories.

Two embeddings $f, g: N \rightarrow \mathbb{R}^{m}$ are said to be (ambient) isotopic (Figure 1.1), if there exists a homeomorphism onto (an ambient isotopy) $F: \mathbb{R}^{m} \times I \rightarrow \mathbb{R}^{m} \times I$ such that

$F(y, 0)=(y, 0)$ for each $y \in \mathbb{R}^{m}$,

$F(f(x), 1)=g(x)$ for each $x \in N, \quad$ and

$F\left(\mathbb{R}^{m} \times\{t\}\right)=\mathbb{R}^{m} \times\{t\} \quad$ for each $t \in I$.
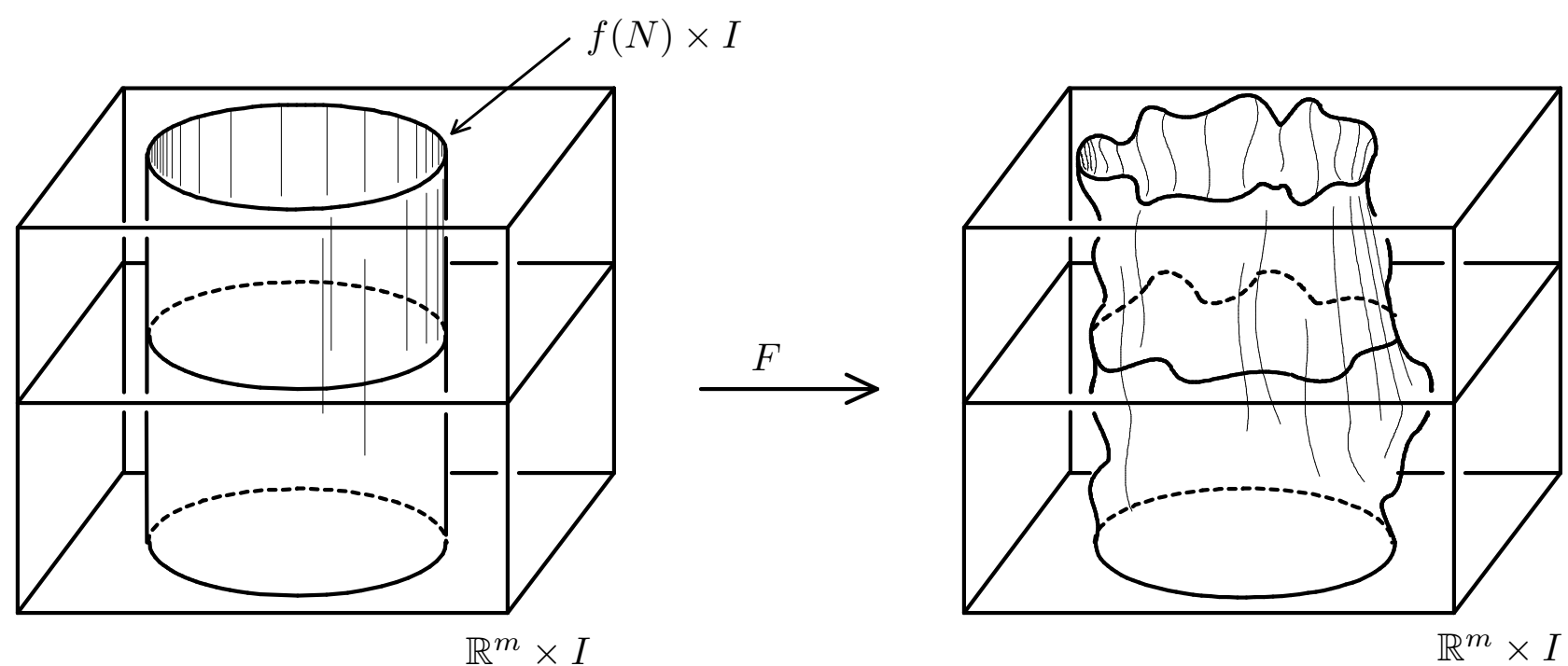

Figure 1.1

An ambient isotopy is also a homotopy $\mathbb{R}^{m} \times I \rightarrow \mathbb{R}^{m}$ or a family of maps $F_{t}: \mathbb{R}^{m} \rightarrow$ 
$\mathbb{R}^{m}$, generated by the map $F$ in the obvious manner. Evidently, ambient isotopy is an equivalence relation on the set of embeddings of $N$ into $\mathbb{R}^{m}$. An embedding is called trivial, if it is isotopic to the standard embedding (the latter is evidently defined from the context).

We use the notations of [RS72]. An equality between sets denotes a 1-1 correspondence. Denote by $V_{m, n}$ the Stiefel manifold of $n$-frames in $\mathbb{R}^{m}$. Let $\mathbb{Z}_{(k)}$ be $\mathbb{Z}$ for $k$ even and $\mathbb{Z}_{2}$ for $k$ odd. Note that $\mathbb{Z}_{(k)}=\pi_{k}\left(V_{n, n-k}\right)$ for $1<k<n$. If the coefficients are omitted from the notation of (co)homology groups, then $\mathbb{Z}$-coefficients are assumed. For a manifold or a polyhedron $N$ we denote its dimension by $n=\operatorname{dim} N$. Denote by $\operatorname{Emb}_{C A T}^{m}(N)$ the set of CAT embeddings $N \rightarrow \mathbb{R}^{m}$ up to ambient CAT isotopy. If $\left|\operatorname{Emb}_{C A T}^{m}(N)\right|=1$, we shall say that $N$ CAT unknots in $\mathbb{R}^{m}$. An embedding is trivial if it is isotopic to the standard embedding (whose choice is clear). For a map $f: N \rightarrow \mathbb{R}^{m}$ we denote by $\Sigma(f)=\operatorname{Cl}\left\{x \in N:\left|f^{-1} f x\right| \geq 2\right\}$ its self-intersection set.

A closed manifold $N$ is called homologically $k$-connected, if $N$ is connected and $H_{i}(N)=$ 0 for each $i=1, \ldots, k$. This condition is equivalent to $\tilde{H}_{i}(N)=0$ for each $i=0, \ldots, k$, where $\tilde{H}_{i}$ are reduced homology groups. A pair $(N, \partial N)$ is called homologically $k$-connected, if $H_{i}(N, \partial N)=0$ for each $i=0, \ldots, k$. Note that if $H_{0}(N, \partial N)=0$, then the manifold $N$ has no closed connected components. We use the following conventions: 0 -connectedness is equivalent to homological 0 -connectedness and to connectedness; $k$-connectedness for $k<0$ is an empty condition. We put $\pi_{l}^{S}=0$ for $l<0$.

Other equivalence relations and problems. Ambient isotopy is a stronger equivalence relation than non-ambient isotopy, isoposition, concordance, bordism, etc. Two embeddings $f, g: N \rightarrow \mathbb{R}^{m}$ are called (non-ambient) isotopic, if there exists an embed$\operatorname{ding} F: N \times I \rightarrow \mathbb{R}^{m} \times I$ such that

$F(x, 0)=(f(x), 0)$,

$F(x, 1)=(g(x), 0)$ for each $x \in N$ and

$F(N \times\{t\}) \subset \mathbb{R}^{m} \times\{t\}$ for each $t \in I$.

In the DIFF category or for $m-n \geq 3$ in the PL (or TOP) category isotopy implies ambient isotopy [HZ64, Hu66, Ak69, Ed75, §7]. For $m-n \leq 2$ this is not so: e.g., any knot $S^{1} \rightarrow S^{3}$ is non-ambiently PL isotopic to the trivial one, but not necessarily ambiently PL isotopic to it.

Two embeddings $f, g: N \rightarrow \mathbb{R}^{m}$ are said to be (orientation preserving) isopositioned, if there is an (orientation preserving) homeomorphism $h: \mathbb{R}^{m} \rightarrow \mathbb{R}^{m}$ such that $h \circ f=g$. For embeddings into $\mathbb{R}^{m}$ orientation preserving isoposition is equivalent to isotopy (the Alexander-Guggenheim Theorem) [RS72, 3.22].

Two embeddings $f, g: N \rightarrow \mathbb{R}^{m}$ are said to be ambiently concordant if there is a homeomorphism (onto) $F: \mathbb{R}^{m} \times I \rightarrow \mathbb{R}^{m} \times I$ (which is called a concordance) such that

$F(y, 0)=(y, 0)$ for each $y \in \mathbb{R}^{m}$ and

$F(f(x), 1)=g(x)$ for each $x \in N$.

The definition of non-ambient concordance is analogously obtained from that of nonambient isotopy by dropping the last condition of level-preservation. In the DIFF category or for $m-n \geq 3$ in the PL (or TOP) category concordance implies ambient concordance and isotopy [Li65, Hu70, HL71] (this is not so in codimension 2). This result allows a reduction of the problem of isotopy to the relativized problem of embeddability.

Let us give a (by no means complete) list of references for closely related problems in geometric topology (the references inside the papers listed here could also be useful for a 
reader). In the problems of embeddability and isotopy the space $\mathbb{R}^{m}$ can be replaced by an arbitrary space $Y$. The case when $Y$ is a manifold has been studied most extensively; for the case when $Y$ is a product of trees see [St89, Theorem 4.6 and Remark, GR92, GMR94, Zh94, Ku00]. For embeddings up to cobordism see [Br71, Li75]. For embeddings up to homotopy see [Co69, St, Wa70, §11, Hu70', CW78, Ha84]. For the classification of link maps see [Mi54, MR86, Ko88, Ko90, Ma90, HK98, Sk00]. For embeddings of polyhedra in some manifolds see [Wa66, Ne68, LS69, RBS99]. For the problem of embeddability of compacta and the close problem of approximability by embeddings see [Ch69, Mc67, Si69, SS83, KW85, Da86, Ak96, Ak96', RS96, §9, Mi97, RS98, Ak00, RS01', ARS02, Me02, RS02, Sk03', Me04] (the author is grateful to P. Akhmetiev for indicating that the paper [Ak96] contains a mistake for $n=3,7$ and that the paper [Ak96'] contains a 'preliminary version' of the proof, the complete version being submitted elsewhere). For the problem of intersection of compacta see [DRS93, ST91]. For basic embeddings see [St89, Sk95, RS99, $\S 5, \mathrm{Ku} 00]$. For immersions see [Gi71, Ad93, cf. Sk02].

Acknowledgements. This survey was based on lectures the author had given at various times at the Independent University of Moscow, Moscow State University, the Steklov Mathematical Institute (Moscow and St. Petersburg branches), the Technical University of Berlin, the Ruhr University of Bochum, the Lorand Eötvos University of Budapest, the University of Geneva, the University of Heidelberg, the University of Ljubljana, the University of Siegen, the University of Uppsala, the University of Warsaw and the University of Zagreb. The preliminary version was prepared in January 2002 after a series of lectures at the Universities of Aberdeen, Cambridge, Edinburgh and Manchester, sponsored by London Mathematical Society. I would like to acknowledge all these institutions for their hospitality and, personally, P. M. Akhmetiev, V. M. Buchstaber, A. V. Chernavskiy, P. Eccles, K. E. Feldman, A. T. Fomenko, M. Kreck, U. Koschorke, R. Lickorish, A. Haefliger, R. Levy, S. Mardesic, A. S. Mischenko, N. Yu. Netsvetaev, V. M. Nezhinskiy, M. M. Postnikov, E. Rees, D. Repovs, E. V. Schepin, Yu. P. Solovyov, A. Szücs, V. A. Vassiliev, O. Ya. Viro, C. Weber, M. Weiss, G. Ziegler and H. Zieschang for their invitations and useful discussions. It is a pleasure to express special gratitude to P. Eccles for his many remarks on the preliminary version of this paper.

\section{The SIMPlEST-TO-STATE RESUltS ON EMBEDDINGS}

\section{Embeddings just below the stable range.}

General Position Theorem 2.1.a. Every $n$-polyhedron or $n$-manifold embeds into $\mathbb{R}^{2 n+1}$ and unknots in $\mathbb{R}^{m}$ for $m \geq 2 n+2$.

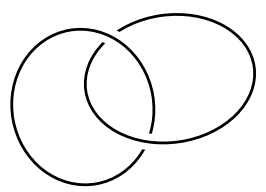

(a)
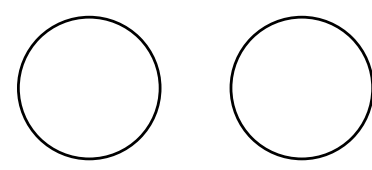

(b)

Figure 2.1

The restriction $m \geq 2 n+2$ in Theorem 2.1.a is sharp as the Hopf linking $S^{n} \sqcup S^{n} \rightarrow$ $\mathbb{R}^{2 n+1}$ shows (Figure 2.1). The number $2 n+1$ in Theorem 2.1.a is the minimal possible for polyhedra. 
Example 2.1.b. For each $n$ there exists an n-polyhedron, non-embeddable in $\mathbb{R}^{2 n}$.

In Example 2.1.b one can take the $n$-th power of a non-planar graph [Sk03], the $n$ skeleton of a $(2 n+2)$-simplex [Ka32, Fl34] or the $(n+1)$-th join power of the three-point set (see the proof for this case in $§ 5$ ).

Note that

$N \times I$ embeds into $\mathbb{R}^{2 n+1}$ for each $n$-polyhedron $N$ [RSS95];

$N \times I$ unknots in $\mathbb{R}^{2 n+2}$ for each $n$-polyhedron $N$ (let us sketch a proof which can though be omitted for the first reading: the result follows because by general position every two embeddings $N \times I \rightarrow \mathbb{R}^{2 n+2}$ are regular homotopic and their restrictions to the spine $N \times\left\{\frac{1}{2}\right\}$ are isotopic).

Theorem 2.2.a. Every $n$-manifold embeds into $\mathbb{R}^{2 n}$ [Ka32, Wh44].

Theorem 2.2.a (as well as Theorem 2.2.b below) is proved using general position and the Whitney trick; the proof in the smooth and PL case is sketched in $\S 4$ and in [RS72, RS99, §8], respectively.

The dimension $2 n$ in Theorem 2.2.a is the best possible when $n=2^{k}$ because $\mathbb{R} P^{2^{k}}$ does not embed into $\mathbb{R}^{2^{k+1}-1}$ (this is proved using the mod2 Whitney obstruction defined below [MS74, RS00, RS02']). But is not the best possible for other $n$ by Theorem 2.3 below. A celebrated and difficult conjecture is that every closed $n$-manifold embeds into $\mathbb{R}^{2 n+1-\alpha(n)}$, where $\alpha(n)$ is the number of units in the dyadic expansion of $n$. The analogous conjecture for immersions was proved in [La82, Co85]. Note that if $n=2^{k_{1}}+\cdots+2^{k_{\alpha(n)}}$ and $k_{1}<\cdots<k_{\alpha(n)}$, then the $n$-manifold $N=\mathbb{R} P^{2^{k_{1}}} \times \cdots \times \mathbb{R} P^{2^{k_{\alpha}(n)}}$ does not embed into $\mathbb{R}^{2 n-\alpha(n)}$ (this is proved again using the mod2 Whitney obstruction defined below).

Theorem 2.3. (a) Every $n$-manifold (except that if $n=2^{k}$ and the manifold is closed, we need to assume that it is orientable) embeds into $\mathbb{R}^{2 n-1}$.

(b) Suppose that $N$ is a closed $n$-manifold, $n$ is even, $n \neq 2^{k}\left(2^{h}+1\right)$ for integers $k, h \geq 2$ and $H_{1}(N)=0$. Then $N$ embeds into $\mathbb{R}^{2 n-2}$, provided $n \geq 6$ in the PL category or $n \geq 8$ in the smooth category.

(c) Suppose that $N$ is a closed $n$-manifold, $\alpha(n) \geq 5$ and either $n=0,1(4)$ and $N$ is orientable, or $n=2,3(4)$ and $N$ is non-orientable. Then $N$ embeds into $\mathbb{R}^{2 n-2}$.

Classical Theorems 2.3 are much more complicated to prove than Theorem 2.2.a: one needs a generalization of the Whitney trick and calculation of characteristic classes, both non-trivial. Theorems 2.3.ab follow from the case $d=0$ of Theorem 2.8.a below (which is true for orientable manifolds) and [Ma60, Theorem 1.c and Corollary 2, Ma62, Theorem 1], except that Theorem 2.3.a for $n=3,4$ has to be proved separately [Hi61, Hi65, Ro65, Wa65, BH70, Do87, Fu94, cf. No61, Fu02]. (From the proof [Ma60, p. 100] it follows that the restriction [Ma60, Theorem 1.c] should be stated as 'the number of $h_{i}$ 's which are equal to $h_{q}+1$ is even', cf. [Hi61].) Theorem 2.3.c follows from the Haefliger-Weber Theorem 5.4 below and [Ba75, Theorem 45].

The condition ' $\alpha(n) \geq 5$ ' in Theorem 2.3.c can be weakened to ' $n \geq 7$ and $\bar{w}_{n-i}(N)=0$ for each $i \leq 4$ ' (the classes $\bar{w}_{i}(N)$ are defined in the subsection 'the Whitney obstruction' below).

Theorem 2.2.b. Every connected $n$-manifold unknots in $\mathbb{R}^{2 n+1}$ for $n>1$ [Wu58].

Here for each $n$ the dimension $2 n+1$ is the best possible and the connectedness assumption is indeed necessary, as the Hopf linking above and the Hudson Torus Example 
2.6.c below show. The Hopf linking is distinguished from the standard linking using the simplest ( $\mathbb{Z}$-valued or $\mathbb{Z}_{2}$-valued) linking coefficient (whose definition is obtained from the definition of the Whitney invariant by setting $m=2 n+1$ in the subsection 'the Whitney invariant' below).

Theorem 2.4. Suppose that $n \geq 2$ and a compact $n$-manifold $N$ has $s$ closed orientable connected components and $t$ closed non-orientable connected components (and, possibly, some non-closed components). Then the set of pairwise linking coefficients defines a 1-1 correspondence $\mathrm{Emb}^{2 n+1}(N) \rightarrow \mathbb{Z}^{\frac{s(s-1)}{2}} \oplus \mathbb{Z}_{2}^{s t+\frac{t(t-1)}{2}}$.

Note that every n-polyhedron $N$ such that $H^{n}(N)=0$

(a) $P L$ (if $n=2$, only $T O P$ ) embeds into $\mathbb{R}^{2 n}$;

(b) PL unknots in $\mathbb{R}^{2 n+1}$.

Assertion (a) for $n \geq 3$ is deduced from Theorems 4.1 or 5.4 below [Wu58, We68, see also Ho71]. For $n=2$ it was proved independently [Ki84] and for $n=1$ it is trivial. Assertion (b) for $n \geq 2$ is deduced from Theorems 4.4 or 5.4 below [Pr66] and for $n=1$ it is trivial. In these assertions for each $n$ the dimensions are the best possible and the $H^{n}(N)=0$ assumption is indeed necessary.

\section{Embedding and unknotting of highly-connected manifolds.}

Theorem 2.5. The sphere $S^{n}$, or even any homology n-sphere,

(a) PL unknots in $\mathbb{R}^{m}$ for $m-n \geq 3$ [Ze60, St63, Gl63, Sc77];

(b) DIFF unknots in $\mathbb{R}^{m}$ for $m \geq \frac{3 n}{2}+2$ [Ha61, Ha62", Ad93, §7];

(c) PL (if $n=3$, only TOP) embeds into $\mathbb{R}^{n+1}$ [follows from Ke69] and

(d) DIFF embeds into $\mathbb{R}^{[3 n / 2]+2}$ [Ha61, Ha62", Ad93, §7].

Theorem 2.5.a is also true in the TOP locally flat category (see the definition in $\S 3$ ) [Ru73, Sc77]. Here the local flatness assumption is indeed necessary.

Knots in codimension 2 and the Trefoil Knot Example 3.5 below show that the dimension restrictions are sharp (even for standard spheres) in Theorems 2.5.a and 2.5.b, respectively. By [Le65, HLS65, cf. MT95, pp. 407-408] the dimension restriction in Theorem 2.5.d is indeed necessary (and conjecturally almost sharp) even for homotopy spheres. However, from [Bo71], it follows that

any $4 k$-dimensional homotopy sphere embeds into $\mathbb{R}^{6 k+1}$.

Theorems 2.2.b and 2.5 may be generalized as follows.

Theorem 2.6. For $n \geq 2 d+2$, every closed homologically $d$-connected $n$-manifold

(a) embeds into $\mathbb{R}^{2 n-d}(n \neq 2 d+2$ in the DIFF case) and

(b) unknots in $\mathbb{R}^{2 n-d+1}$.

Theorem 2.6 was proved directly in [PWZ61, Ha61, Ze62, Ir65, Hu69] for homotopically $d$-connected manifolds and using the deleted product method ( $\$ 5)$ in [Ha62", We67, Ad93, §7, Sk97, Sk02] for homologically d-connected manifolds. Theorem 2.6 follows from Theorem 2.8 below. Note that if $n \leq 2 d+1$, then every closed homologically $d$-connected $n$-manifold is a homology sphere, so the PL case of Theorem 2.6 gives nothing more than Theorem 2.5.

For generalizations of Theorem 2.6 see Theorem 2.8 below or [Hu67, Ha68', Hu72, Go72, Ke79]. We shall use of them (the simplest case $2 m \geq 3 n+3$ of) the following relative version of Theorem 2.6.a. 
The Penrose-Whitehead-Zeeman-Irwin Embedding Theorem 2.6.c. If $m$ $n \geq 3$, then any proper map from a $(2 n-m)$-connected $P L n$-manifold with boundary to a $(2 n-m+1)$-connected $P L m$-manifold with boundary, whose restriction to the boundary is an embedding, is homotopic (relatively to the boundary) to a PL embedding [PWZ61, Ir65].

The dimension assumption in Theorem 2.6.b is sharp:

The Hudson Torus Example 2.6.c. For each $p \leq q$ there exists a non-trivial embedding $S^{p} \times S^{q} \rightarrow \mathbb{R}^{p+2 q+1}[\mathrm{Hu} 63]$.

Simplified construction [Sk06]. (This construction is interesting even for $p=q=$ 1!) Take the standard embedding $2 D^{p+q+1} \times S^{q} \subset \mathbb{R}^{p+2 q+1}$. The Hudson torus is the (linked!) connected sum of the $(p+q)$-sphere $2 \partial D^{p+q+1} \times x$ with the standard embedding $\partial D^{p+1} \times S^{q} \subset D^{p+q+1} \times S^{q} \subset \mathbb{R}^{p+2 q+1}$.

The Hudson torus can be distinguished from the standard embedding using the Whitney invariant defined in the subsection under the same name below [Sk06] or the Haefliger-Wu invariant defined in $\S 5[\mathrm{Sk} 02]$.

The rest of this subsection can be omitted for the first reading.

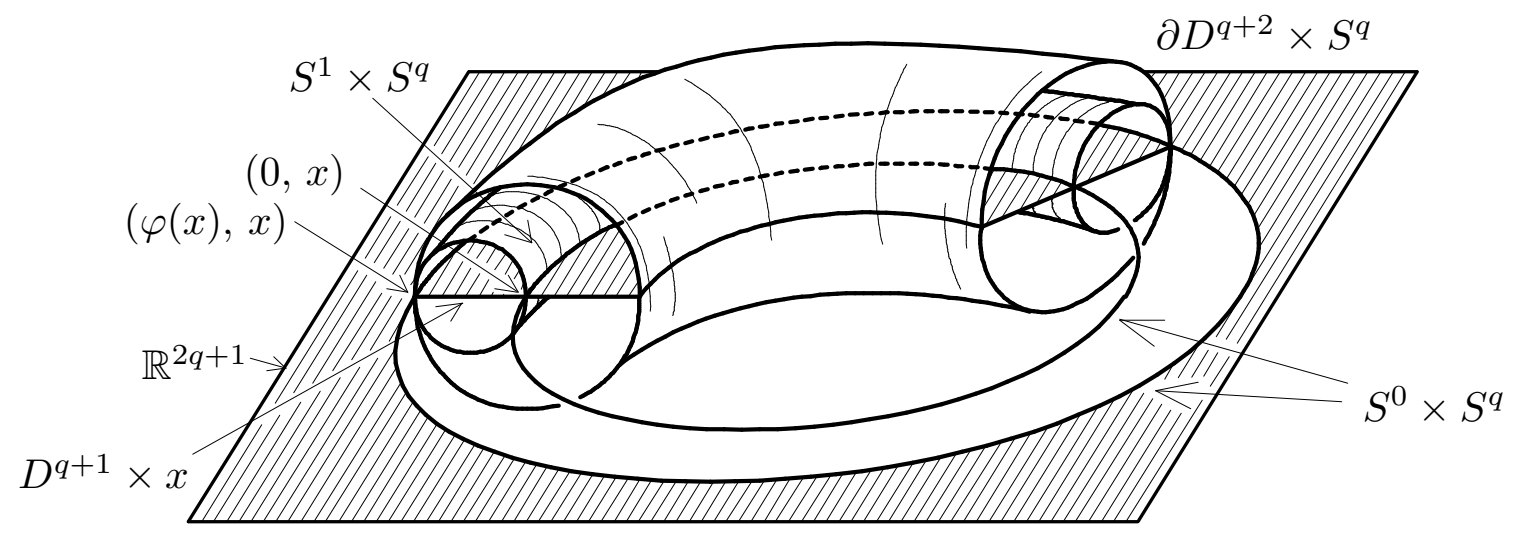

Figure 2.2.a

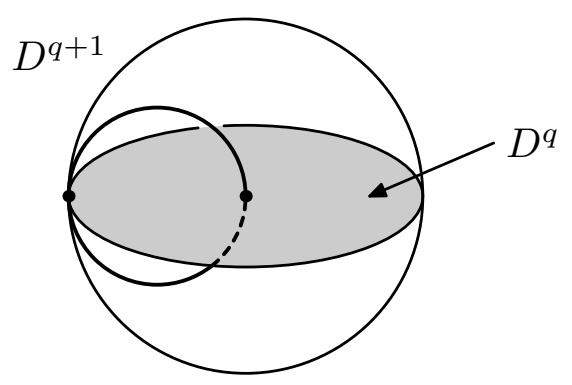

Figure 2.2.b

An alternative simplified construction of the Hudson torus [Sk02, Sk]. Define a map $S^{0} \times S^{q} \rightarrow D^{q+1}$ to be the constant $0 \in D^{q+1}$ on one component and the standard embedding $\varphi$ on the other component. This map gives an embedding

$$
S^{0} \times S^{q} \rightarrow D^{q+1} \times S^{q} \subset D^{q+2} \times S^{q} \subset \mathbb{R}^{2 q+2}
$$


(Figure 2.2.a). Each disk $D^{q+2} \times x$ intersects the image of this embedding at two points lying in $D^{q+1} \times x$. Extend this embedding $S^{0} \rightarrow D^{q+1} \times x$ for each $x \in S^{q}$ to an embedding $S^{1} \rightarrow D^{q+2} \times x$ (Figure 2.2.b). Thus we obtain a non-standard embedding

$$
S^{1} \times S^{q} \rightarrow D^{q+2} \times S^{q} \subset \mathbb{R}^{2 q+2} .
$$

Taking spheres of dimensions $p \leq q$ we obtain analogously an embedding

$$
S^{p} \times S^{q} \rightarrow D^{q+p+1} \times S^{q} \subset \mathbb{R}^{2 q+p+1} .
$$

(Taking as $\varphi$ above an arbitrary CAT-map $S^{q} \rightarrow \partial D^{m-q-p}$ we obtain analogously a CAT embedding $S^{p} \times S^{q} \stackrel{\varphi \times \operatorname{pr}_{2}}{\rightarrow} D^{m-q} \times S^{q} \rightarrow \mathbb{R}^{m}$.)

It would be interesting to know whether the smooth case of Theorem 2.6.a holds for $n=2 d+2$, i.e. for $d$-connected $2(d+1)$-manifolds. An almost smooth embedding is a $\mathrm{PL}$ embedding which is a smooth embedding outside a point.

Theorem 2.7. Let $N$ be a closed smooth $(l-1)$-connected $2 l$-manifold.

(a) If $l \equiv 3,5,7 \bmod 8$ and $l \neq 2^{s}-1$, then $N$ almost smoothly embeds into $\mathbb{R}^{2 l+1}$.

(b) For $l$ even the manifold $N$ almost smoothly embeds into $\mathbb{R}^{2 l+1}$ if and only if $N$ is stably parallelizable.

(c) If $N$ is almost parallelizable, then $N$ almost smoothly embeds into $\mathbb{R}^{2 l+2}$ [MRS03].

(d) For each even $l$ there exists a closed smooth $(l-1)$-connected (even almost parallelizable) 2l-manifold which does not smoothly embed into $\mathbb{R}^{3 l-1}$ [MRS03].

Theorems 2.7.a and 2.7.b are proved in [Mi65, Corollary 1, Theorem 2 and Addendum (1)], cf. [Sa65]. By Theorem 2.7.c, the manifold from 2.7.d almost smoothly embeds into $\mathbb{R}^{2 l+2}$.

Proof of 2.7.c. If $l=2$, then the result holds by [Ma78, Corollary 10.11, CS79]. So assume that $l \neq 2$. Let $N_{0}$ be a complement in $N$ to some open $2 l$-ball. Then $N_{0}$ is parallelizable and hence there is an immersion $f: N_{0} \rightarrow \mathbb{R}^{2 l+1}$. Since $N_{0}$ is $(l-1)$ connected and $l \neq 2$, it follows that it has an $l$-dimensional spine [Wa64, Ho69]. By general position the restriction of $f$ to this spine is an embedding. Hence the restriction of $f$ to some neighborhood of this spine is an embedding. But this neighborhood is homeomorphic to $N_{0}$. So there is an embedding $g: N_{0} \rightarrow \mathbb{R}^{2 l+1}$. Extending the embedding $\left.g\right|_{\partial N_{0}}$ as a cone in $\mathbb{R}^{2 l+2}$ we obtain an almost smooth embedding of $N$ into $\mathbb{R}^{2 l+2}$.

Proof of 2.7.d. Take the Kervaire-Milnor closed smooth almost parallelizable $4 k$ manifold $N$ whose signature $\sigma(N) \neq 0$ [Ma80, MK58]. We can modify this by surgery [Ma80] and assume further that it is $(l-1)$-connected. Hence the Pontrjagin class $p_{l / 2}(N, \mathbb{R}) \neq 0$ by the Hirzebruch formula. Therefore $\bar{p}_{l / 2}(N, \mathbb{R}) \neq 0$ by the duality theorem for real Pontryagin classes [Wu65, cf. MS74]. Hence $N$ does not smoothly embed into $\mathbb{R}^{3 l-1}$ (it does not even immerse in $\mathbb{R}^{3 l-1}$ ) [Po42, Wu65].

The dimension $2 n-d$ in Theorem 2.6. a can be decreased by 1 for some pairs $(n, d)$, as Theorem 2.3 shows. However, we conjecture that the dimension $2 n-d$ in Theorem 2.6.a cannot be significantly decreased for some $(n, d)$. This is so for $d=0$ (as the example $N=\mathbb{R} P^{a_{1}} \times \cdots \times \mathbb{R} P^{a_{s}}$ shows) and for $n=2,4,8, d=\frac{n}{2}-1$ (take $N=\mathbb{R} P^{2}, \mathbb{C} P^{2}, \mathbb{H} P^{2}$ or apply Theorem 2.7.d). Example of a highly-connected but badly embeddable manifolds were also exhibited in [HS64, Sa65]. 


\section{The Whitney obstruction.}

Definition of the modulo 2 Whitney obstruction. Let $N$ be a closed manifold. We present the definition in the piecewise linear case, the definition in the (piecewise) smooth case is analogous. Take any general position map $f: N \rightarrow \mathbb{R}^{m}$. Recall the definition of the self-intersection set $\Sigma(f)$ from $\S 1$ (Figure 2.3 ).

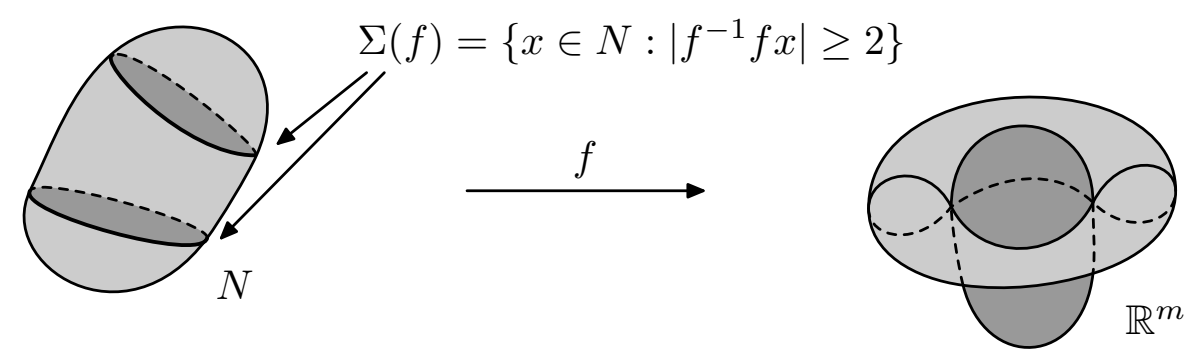

Figure 2.3

Take a triangulation $T$ of $N$ such that $f$ is linear on simplices of $T$. Then the selfintersection set $\Sigma(f)$ is a subcomplex of $T$. Denote by $[\Sigma(f)] \in C_{2 n-m}\left(N ; \mathbb{Z}_{2}\right)$ the sum of the top-dimensional simplices of $\Sigma(f)$ (Figure 2.1). Then $[\Sigma(f)]$ is a cycle [Hu69, Lemma 11.4, Hu70', Lemma 1].

(In order to prove this fact it suffices to prove that each $(2 n-m-1)$-simplex $\eta$ of $T$ is in the boundary of an even number of $(2 n-m)$-simplices $\sigma \subset \Sigma(f)$. It suffices to consider the case $\eta \in \Sigma(f)$. By general position, $f^{-1} f \eta$ consists of simplices $\eta=\eta_{1}, \ldots, \eta_{k}$. The link $\mathrm{lk}_{T} \eta_{i}$ is a sphere of dimension $n-(2 n-m-1)-1=m-n$. The link $\mathrm{lk}_{\mathbb{R}^{m}} f \eta$ is a sphere of dimension $m-(2 n-m-1)-1=2(m-n)$. The intersection of two $f$-images $f\left(\mathrm{lk}_{T} \eta_{i}\right)$ of $(m-n)$-spheres in the $2(m-n)$-sphere $\mathrm{lk}_{\mathbb{R}^{m}} f \eta$ consists of an even number of points. These intersection points are in 1-1 correspondence with $(2 n-m)$-simplices $\sigma \subset \Sigma(f)$ containing $\eta$ in their boundaries.)

The modulo 2 Whitney obstruction is the homology class

$$
\bar{w}_{m-n}(N):=[\Sigma(f)] \in H_{2 n-m}\left(N ; \mathbb{Z}_{2}\right) .
$$

The class $\bar{w}_{i}$ is called the normal Stiefel-Whitney class. This definition of the normal Stiefel-Whitney classes is equivalent to other definitions [MS74], up to Poincaré duality.

The independence of $\bar{w}_{m-n}(N)$ on $f$ follows from the equality $\left[\Sigma\left(f_{0}\right)\right]-\left[\Sigma\left(f_{1}\right)\right]=$ $\partial[\Sigma(F)]$ for a general position homotopy $F: N \times I \rightarrow \mathbb{R}^{m} \times I$ between general position $\operatorname{maps} f_{0}, f_{1}: N \rightarrow \mathbb{R}^{m}$.

Hence these classes are obstructions to the embeddability of $N$ into $\mathbb{R}^{m}$ :

if $N$ embeds into $\mathbb{R}^{m}$, then $\bar{w}_{i}(N)=0$ for $i \geq m-n$ [Wh35].

Definition of the Whitney obstruction for $N$ orientable and $m-n$ odd. Fix in advance any orientation of $N$ and of $\mathbb{R}^{m}$. The definition is analogous to the above, only $[\Sigma(f)]$ is the sum of oriented simplices $\sigma$ with \pm signs defined as follows. (For $m-n$ even the signs can also be defined but are not used because $[\Sigma(f)]$ is not necessarily a cycle with integer coefficients).

By general position there is a unique simplex $\tau$ of $T$ such that $f(\sigma)=f(\tau)$. The orientation on $\sigma$ induces an orientation on $f \sigma$ and then on $\tau$. The orientations on $\sigma$ and $\tau$ induce orientations on normal spaces in $N$ to these simplices. These two orientations (in this order) together with the orientation on $f \sigma$ induce an orientation on $\mathbb{R}^{m}$. If 
this orientation agrees with the fixed orientation of $\mathbb{R}^{m}$, then the coefficient of $\sigma$ is +1 , otherwise -1 . Clearly, the change of orientation of $\sigma$ changes the sign of $\sigma$ in $[\Sigma(F)]$, so the sign is well-defined.

(An equivalent definition of the signs in $[\Sigma(f)]$ is as follows. The orientation on $\sigma$ induces an orientation on $f \sigma$ and then on $\tau$, hence it induces an orientation on their links. Consider the oriented sphere $\operatorname{lk}_{\mathbb{R}^{m}} f \sigma$, that is the link of $f \sigma$ in certain triangulation of $\mathbb{R}^{m}$ 'compatible' with $T$. The dimension of this sphere is $m-1-(2 n-m)=2(m-n)-1$. This sphere contains disjoint oriented $(m-n-1)$-spheres $f\left(\mathrm{lk}_{T} \sigma\right)$ and $f\left(\mathrm{lk}_{T} \tau\right)$. The coefficient of $\sigma$ in $[\Sigma(F)]$ is their linking coefficient, which equals \pm 1 .)

The Whitney obstruction is the homology class

$$
\bar{W}_{m-n}(N):=[\Sigma(f)] \in H_{2 n-m}(N ; \mathbb{Z})
$$

Clearly, $\bar{w}_{i}(N)$ are modulo 2 reductions of $\bar{W}_{i}(N)$. The classes $\bar{w}_{i}(N)$ are easier to compute, however they are possibly weaker than $\bar{W}_{i}$.

Pontryagin introduced for closed orientable $n$-manifold $N$ the Pontryagin classes $\bar{p}_{i} \in$ $H_{n-4 i}(N ; \mathbb{Z})$ which obstruct to embeddability into $\mathbb{R}^{n+2 i-1}[\mathrm{Po} 42]$.

Recall the definition of $\mathbb{Z}_{(k)}$ from $\S 1$. For a closed orientable $n$-manifold $N$ denote by $\bar{W}_{m-n}(N) \in H_{2 n-m}\left(N, \mathbb{Z}_{(m-n-1)}\right)$ the class $\bar{W}_{m-n}(N)$ for $m-n$ odd and the class $\bar{w}_{m-n}(N)$ for $m-n$ even.

Theorem 2.8.a. Let $N$ be a closed d-connected $n$-manifold, $d \geq 1$. The manifold $N$ embeds into $\mathbb{R}^{2 n-d-1}$ if $\bar{W}_{n-d-1}(N)=0$, provided $n \geq d+4$ or $n \geq 2 d+5$, in the PL or DIFF cases respectively.

See references to proofs after Theorem 2.8.b.

In Theorem 2.8.a the $d$-connectedness assumption can be weakened to the homological $d$-connectedness except when $n=2 d+2$ in the PL case. The PL case of Theorem 2.8.a gives nothing but Theorem 2.5.c for $d+4 \leq n \leq 2 d+1$. The smooth case of Theorem 2.8. a is true if $d$ is even and $n=2 d+3$ [Sk02, Corollary 1.7].

\section{The Whitney invariant.}

Definition of the Whitney invariant. Let $N$ be a closed connected orientable $n$-manifold. Let $f_{0}: N \rightarrow \mathbb{R}^{m}$ be a certain fixed ('standard') embedding and let $f: N \rightarrow \mathbb{R}^{m}$ be an arbitrary embedding. Take a general position homotopy $F: N \times I \rightarrow \mathbb{R}^{m} \times I$ between $f$ and $f_{0}$ (Figure 2.4).

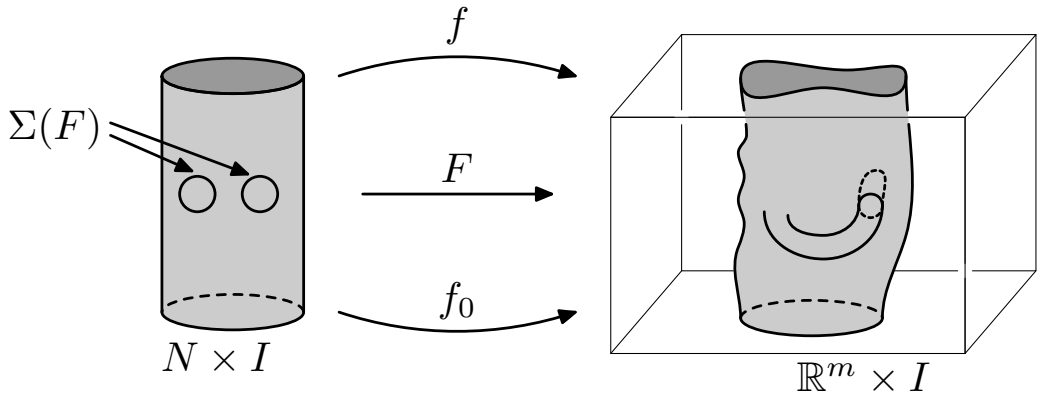

Figure 2.4

Analogously to the above, the self-intersection set $\Sigma(F)$ supports a $(2 n-m+1)$-cycle $[\Sigma(F)]$ in $N \times I \simeq N$ with the coefficients $\mathbb{Z}_{(m-n-1)}$. The Whitney invariant of $f$ is the 
homology class of this cycle:

$$
W(f):=[\Sigma(F)] \in H_{2 n-m+1}\left(N, \mathbb{Z}_{(m-n-1)}\right) .
$$

Analogously to the above, $W(f)$ depends only on $f$ and $f_{0}$ but not on the choice of $F$ [Hu69, §11, cf. HH63, Vr77, Sk06].

Theorem 2.8.b. Let $N$ be a closed orientable homologically d-connected n-manifold, $d \geq 0$. Then the Whitney invariant

$$
W: \operatorname{Emb}^{2 n-d}(N) \rightarrow H_{d+1}\left(N, \mathbb{Z}_{(n-d-1)}\right)
$$

is a bijection, provided $n \geq d+3$ or $n \geq 2 d+4$, in the PL or DIFF cases respectively.

Theorems 2.8.a and 2.8.b were proved in [Le62, HH63, Hu69, §11, BH70, Bo71, Vr77] directly for homotopically $d$-connected manifolds (except the PL case of Theorem 2.8.a) and using the deleted product method ( $\$ 5$ ) in [Ha62", We67, Sk97, Sk02] for homologically $d$-connected manifolds. (The author is grateful to J. Boechat for indicating that [Bo71, Theorem 4.2] needs a correction; this does not affect the main result of [Bo71].)

E.g. by Theorem 2.8.b we obtain that the Whitney invariant $W: \operatorname{Emb}^{p+2 q+1}\left(S^{p} \times\right.$ $\left.S^{q}\right) \rightarrow \mathbb{Z}_{(q)}$ is bijective for $1 \leq p \leq q-2$, cf. Theorem 3.8 below. The generator is the Hudson torus.

The PL case of Theorem 2.8.b gives nothing but Theorem 2.5.a for $d+3 \leq n \leq 2 d+1$.

Analogously to Theorem 2.8.b it may be proved that if $N$ is a closed connected nonorientable $n$-manifold, then

$$
\operatorname{Emb}^{2 n}(N)= \begin{cases}H_{1}\left(N, \mathbb{Z}_{2}\right) & n \text { odd, } \\ \mathbb{Z} \oplus \mathbb{Z}_{2}^{s-1} & n \text { even and } H_{1}\left(N, \mathbb{Z}_{2}\right) \cong \mathbb{Z}_{2}^{s},\end{cases}
$$

provided $n \geq 3$ or $n \geq 4$, in the PL or DIFF case respectively [Ba75, Vr77]. (There is a mistake in the calculation for the non-orientable case in [Ha62", We67, Theorem B].)

Because of the existence of knots the analogues of Theorem 2.8.b for $n=d+2$ in the PL case, and for (most) $n \leq 2 d+3$ in the smooth case are false. So for the smooth category and $n \leq 2 d+3$ a classification is much harder: until recently the only known concrete complete classification results were for spheres and their disjoint unions. Recently the following two results were obtained using the Kreck modification of surgery theory.

Theorem 2.9. [Sk06] Let $N$ be a closed parallelizable homologically $(2 k-2)$-connected $(4 k-1)$-manifold. Then the Whitney invariant $W: \operatorname{Emb}_{D I F F}^{6 k}(N) \rightarrow H_{2 k-1}(N)$ is surjective and for each $u \in H_{2 k-1}(N)$ there is a 1-1 correspondence $\eta_{u}: W^{-1} u \rightarrow \mathbb{Z}_{d(u)}$, where $d(u)$ is the divisibility of the projection of $u$ to $H_{1}(N) /$ Tors.

Recall that the divisibility of zero is zero and the divisibility of $x \in G-\{0\}$ is $\max \{d \in$ $\mathbb{Z} \mid$ there is $\left.x_{1} \in G: x=d x_{1}\right\}$. E.g. by Theorem 2.9 we obtain that the Whitney invariant $W: \mathrm{Emb}^{6 k}\left(S^{2 k-1} \times S^{2 k}\right) \rightarrow \mathbb{Z}$ is surjective and for each $u \in \mathbb{Z}$ there is a $1-1$ correspondence $W^{-1} u \rightarrow \mathbb{Z}_{u}$.

Theorem 2.10. [KS05] (a) Let $N$ be a closed connected smooth 4-manifold such that $H_{1}(N)=0$ and the signature $\sigma(N)$ of $N$ is free of squares (i.e. is not divisible by a square of an integer $s \geq 2)$. Then the Whitney invariant $W: \operatorname{Emb}_{D I F F}^{7}(N) \rightarrow H_{2}(N)$ is 
injective. There exists $x_{0} \in H_{2}(N)$ such that $x_{0}^{2}=\sigma(N)$ and $x_{0} \bmod 2=w_{2}(N)$; then $\operatorname{im} W=\left\{y \in H_{2}(N) \mid y^{2}+y \cap x_{0}=0\right\}$.

(b) Let $N$ be a closed simply-connected smooth 4-manifold embeddable into $S^{6}$. Take a composition $f: N \rightarrow S^{6} \subset S^{7}$ of an embedding and the inclusion. Then $\# W^{-1} W(f)=$ 12.

E.g. by Theorem 2.10.a we obtain that $\operatorname{Emb}_{D I F F}^{7}\left(\mathbb{C} P^{2}\right)$ is in $1-1$ correspondence with $\{+1,-1\} \in \mathbb{Z} \cong H_{2}\left(\mathbb{C} P^{2} ; \mathbb{Z}\right)$. One can check that +1 corresponds to the standard embedding [BH70, p. 164] and -1 to its composition with mirror symmetry.

Conjecture. Every smooth embedding $S^{1} \times S^{1} \rightarrow \mathbb{R}^{4}$ is PL isotopic to a connected sum of a knot $S^{2} \rightarrow S^{4}$ either with the standard embedding, or with the right Hudson torus, or with the left Hudson torus, or with the composition of Dehn twist along the parallel and the right Hudson torus.

A similar conjecture question can be stated for arbitrary closed 2-manifolds. Cf. [FKV87, FKV88].

\section{Low-dimensional manifolds.}

For relatively low-dimensional manifolds there are the following results not covered by Theorems 2.2, 2.3, 2.6.a and 2.8.a. (We need not specify whether PL or DIFF manifolds are under consideration because every PL manifold of dimension at most 7 is smoothable.)

Theorem 2.11. (a) A closed orientable 4-manifold $N$ PL embeds into $\mathbb{R}^{6}$ if and only if $\bar{w}_{2}(N)=0$ [Ma78, Corollary 10.11, CS79].

(b) A closed orientable 4-manifold smoothly embeds into $\mathbb{R}^{6}$ if and only if $\bar{w}_{2}(N)=0$ and $p_{1}(N)=0$ [Ma78, Corollary 10.11, CS79, Ru82].

(c) Every 2-connected closed 6-manifold is a connected sum of $S^{3} \times S^{3}$ [Sm62, Theorem $B]$ and therefore embeds into $\mathbb{R}^{7}$.

(d) Every closed non-orientable 6-manifold $N$ such that $\bar{w}_{2}(N)=0$ and $\bar{w}_{3}(N)=0 P L$ embeds into $\mathbb{R}^{10}$ [Sk02].

(e) Let $N$ be a closed simply-connected 6-manifold whose homology are torsion free, and $\bar{w}_{2}(N)=0$.

$N$ embeds into $\mathbb{R}^{7}$ if and only if $N$ is a connected sum of copies of $S^{2} \times S^{4}$ and $S^{3} \times S^{3}$;

$N$ smoothly (or PL locally flat) embeds into $\mathbb{R}^{8}$ if and only if $p_{1}(N)=0$;

$N$ smoothly embeds into $\mathbb{R}^{10}$ [Wa66', Theorems 12 and 13].

(f) Every closed homologically 2-connected 7-manifold PL embeds into $\mathbb{R}^{11}$ [Sk97, Sk02].

The embeddability in $\mathbb{R}^{10}$ in Theorem 2.11.e is true also in the PL case, but this is covered by Theorem 2.3.b.

Take the Dold 5-manifold $N$ such that $\bar{w}_{2,3}(N):=\bar{w}_{2}(N) \bar{w}_{3}(N) \neq 0$ and make surgery killing $\pi_{1}(N)$. We obtain a simply connected 5-manifold $N^{\prime}$ with $\bar{w}_{2,3}\left(N^{\prime}\right) \neq 0$, therefore $\bar{w}_{3}\left(N^{\prime}\right) \neq 0$ and hence $N^{\prime}$ does not embed into $\mathbb{R}^{8}$. This remark of Akhmetiev shows that the dimension $2 n-d$ is the minimal in Theorem 2.6. a for $n=5$ and $d=1$.

We conjecture that there exists a 1-connected 6-manifold $N$ with normal Stiefel-Whitney class $\bar{W}_{3}(N) \neq 0$ so that $N$ does not embed into $\mathbb{R}^{9}$, cf. [Wa66', Zh75, Zh89]. 


\section{LINKS AND KNOTTED TORI}

\section{The linking coefficient.}

Definition of the linking coefficient. Fix orientations of $S^{p}, S^{q}, S^{m}$ and $D^{m-p}$. Assume that $m \geq q+3$ and $f: S^{p} \sqcup S^{q} \rightarrow S^{m}$ is an embedding. Take an embedding $g: D^{m-q} \rightarrow S^{m}$ such that $g D^{m-q}$ intersects $f S^{q}$ transversally at exactly one point with positive sign (Figure 3.1). Then the restriction of $g$ to $\partial D^{m-q}$ is an orientation preserving homotopy equivalence $h: S^{m-q-1} \rightarrow S^{m}-f S^{q}$. The induced isomorphism of homotopy groups does not depend on $g$. The linking coefficient is

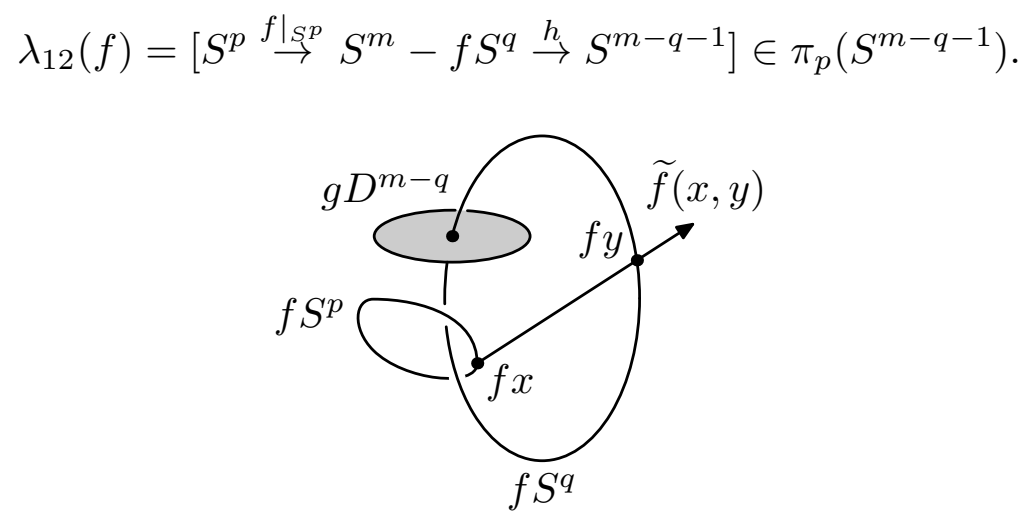

Figure 3.1

Clearly, $\lambda_{12}(f)$ is indeed independent on $h$.

Analogously we may define $\lambda_{21}(f) \in \pi_{q}\left(S^{m-p-1}\right)$ for $m \geq p+3$. The definition works for $m=q+2$ if the restriction of $f$ to $S^{q}$ is PL unknotted (this is always so for $m \geq q+3$ by Theorem 2.5.a). For $m=p+q+1$ there is a simpler alternative definition.

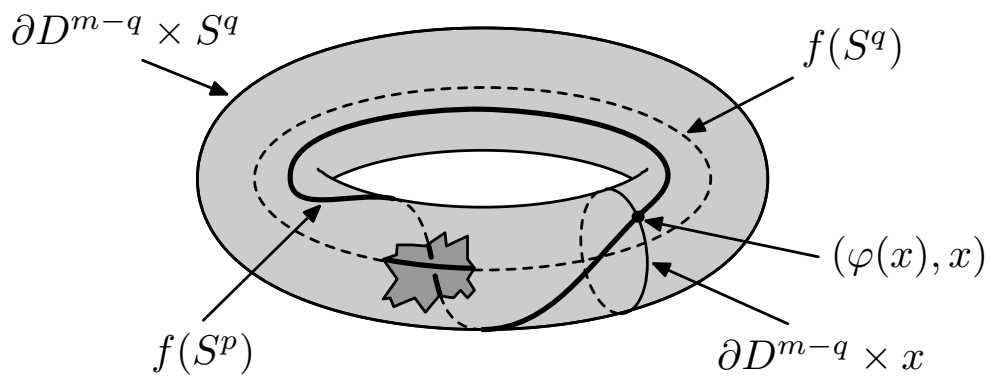

Figure 3.2

Construction of a link with prescribed linking coefficient for $p \leq q \leq m-2$. Define $f$ on $S^{q}$ to be the standard embedding into $\mathbb{R}^{m}$. Take any CAT map $\varphi: S^{p} \rightarrow \partial D^{m-q}$. Define the CAT embedding $f$ on $S^{p}$ by

$$
S^{p} \stackrel{\varphi \times i}{\rightarrow} \partial D^{m-q} \times S^{q} \subset D^{m-q} \times S^{q} \subset \mathbb{R}^{m}
$$

where $i: S^{p} \rightarrow S^{q}$ is the equatorial inclusion and the latter inclusion is the standard. See Figure 3.2. Clearly, $\lambda_{12}(f)=\varphi$.

If $m \geq \frac{p}{2}+q+2$, then $\Sigma^{\infty}: \pi_{p}\left(S^{m-q-1}\right) \rightarrow \pi_{p+q+1-m}^{S}$ is an isomorphism. 
Consider the 'connected sum' commutative group structure on $\operatorname{Emb}^{m}\left(S^{p} \sqcup S^{q}\right)$ defined for $m-3 \geq p, q$ (Figure 3.3) in [Ha66, Ha66'].
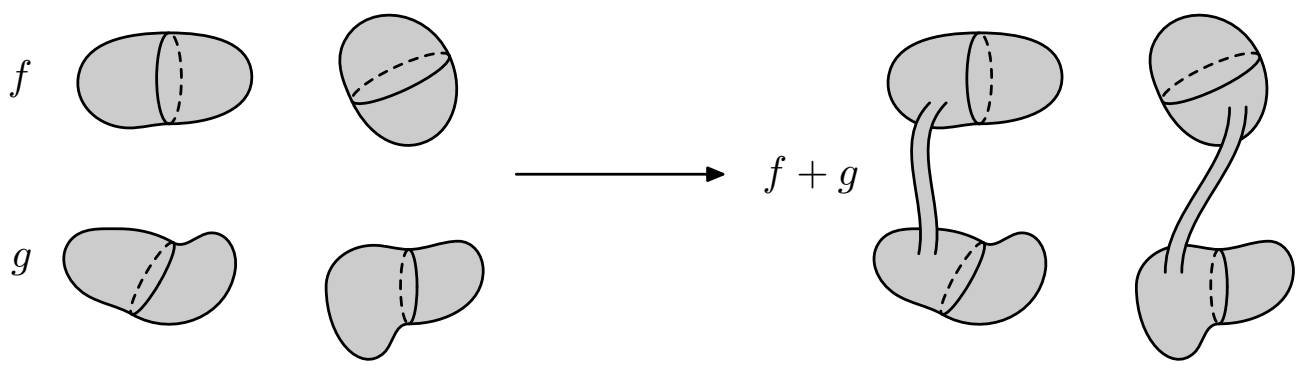

Figure 3.3

The Haefliger-Zeeman Theorem 3.1. If $1 \leq p \leq q$, then the map

$$
\Sigma^{\infty} \lambda_{12}: \operatorname{Emb}^{m}\left(S^{p} \sqcup S^{q}\right) \rightarrow \pi_{p+q+1-m}^{S}
$$

is an isomorphism for $m \geq \frac{p}{2}+q+2$ and for $m \geq \frac{3 q}{2}+2$, in the PL and DIFF cases respectively.

The surjectivity is proved above and does not require the dimension restrictions. The injectivity is proved in [Ha62', Ze62], or follows from the Haefliger-Weber Theorem 5.4 and Deleted Product Lemma 5.3.a below.

By the Haefliger-Zeeman Theorem 3.1 we have the following table for $m \geq \frac{3 q}{2}+2$.

$$
\begin{aligned}
& m \quad: 2 q+2: 2 q+1: 2 q: 2 q-1: 2 q-2: 2 q-3: 2 q-4
\end{aligned}
$$

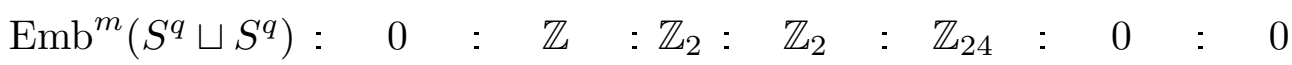

The stable suspension of the linking coefficient can be described alternatively as follows. For an embedding $f: S^{p} \sqcup S^{q} \rightarrow S^{m}$ define a map

$$
\tilde{f}: S^{p} \times S^{q} \rightarrow S^{m-1} \quad \text { by } \quad \tilde{f}(x, y)=\frac{f x-f y}{|f x-f y|}
$$

For $p \leq q \leq m-2$ define the $\alpha$-invariant by

$$
\alpha(f)=[\tilde{f}] \in\left[S^{p} \times S^{q}, S^{m-1}\right] \stackrel{v^{*}}{\cong} \pi_{p+q}\left(S^{m-1}\right) \cong \pi_{p+q+1-m}^{S}
$$

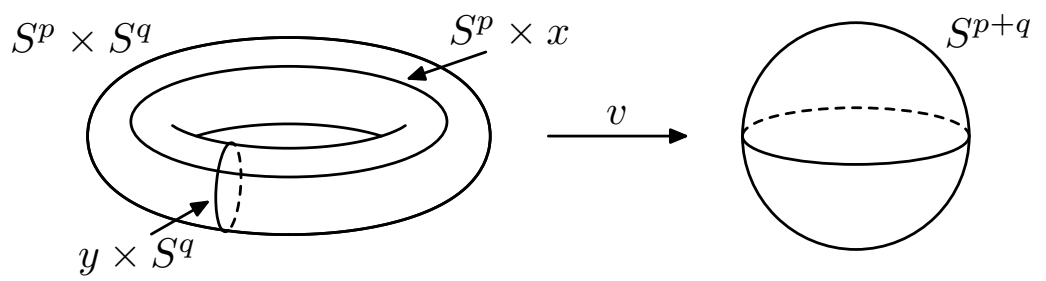

Figure 3.4 
The second isomorphism in the formula for $\alpha(f)$ is given by the Freudenthal Suspension Theorem. The map $v: S^{p} \times S^{q} \rightarrow \frac{S^{p} \times S^{q}}{S^{p} \vee S^{q}} \cong S^{p+q}$ is the quotient map (Figure 3.4). The map $v^{*}$ is an isomorphism for $m \geq q+2$.

(For $m \geq q+3$ this follows by general position and for $m=q+2$ by the cofibration Barratt-Puppe exact sequence of the pair $\left(S^{p} \times S^{q}, S^{p} \vee S^{q}\right)$ and by the existence of a retraction $\Sigma\left(S^{p} \times S^{q}\right) \rightarrow \Sigma\left(S^{p} \vee S^{q}\right)$, cf. [MR86, §3].)

By [Ke59, Lemma 5.1] we have $\alpha= \pm \Sigma^{\infty} \lambda_{12}$.

Note that $\alpha$-invariant can be defined in more general situations [Ko88].

Borromean rings, the Whitehead link and the Trefoil knot. An analogue of the Haefliger-Zeeman Theorem 3.1 holds for links with many components. However, the collection of pairwise $\alpha$-invariants (or even linking coefficients) is not injective for $2 m<3 n+4$ and n-dimensional links with more than two components in $\mathbb{R}^{m}$. This is implied by the following example.

The Borromean Rings Example 3.2. The Borromean rings

$$
S^{2 l-1} \sqcup S^{2 l-1} \sqcup S^{2 l-1} \rightarrow \mathbb{R}^{3 l}
$$

form a non-trivial embedding whose restrictions to 2-componented sublinks are trivial [Ha62, 4.1, Ha62', cf. Ma90].

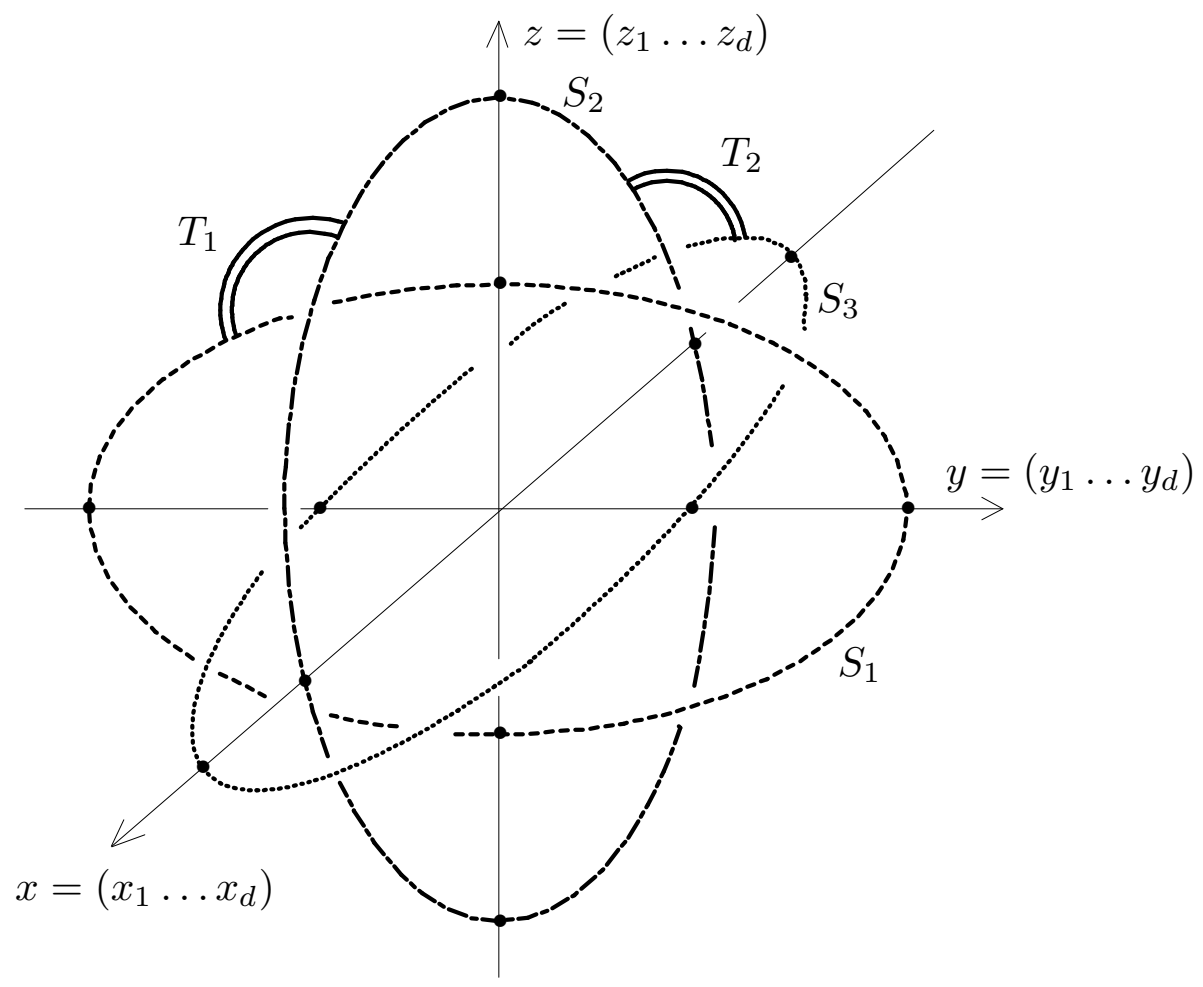

Figure 3.5

Consider the space $\mathbb{R}^{3 l}$ of coordinates

$$
(x, y, z)=\left(x_{1}, \ldots, x_{l}, y_{1}, \ldots, y_{l}, z_{1}, \ldots, z_{l}\right) .
$$


The (higher-dimensional) Borromean rings are three embedded spheres (Figures 3.5, 3.6.b) given by the equations

$$
\left\{\begin{array}{l}
x=0 \\
y^{2}+2 z^{2}=1
\end{array}, \quad\left\{\begin{array} { l } 
{ y = 0 } \\
{ z ^ { 2 } + 2 x ^ { 2 } = 1 }
\end{array} \quad \text { and } \quad \left\{\begin{array}{l}
z=0 \\
x^{2}+2 y^{2}=1
\end{array} .\right.\right.\right.
$$

The following classical example shows that the invariant $\alpha= \pm \Sigma^{\infty} \lambda_{12}: E m b^{m}\left(S^{p} \sqcup\right.$ $\left.S^{q}\right) \rightarrow \pi_{p+q+1-m}^{S}$ can be incomplete for $m<\frac{p}{2}+q+2$ and links with two components, i.e. that the dimension restriction in the Haefliger-Zeeman Theorem 3.1 is sharp.

The Whitehead Link Example 3.3. The Whitehead link $w: S^{2 l-1} \sqcup S^{2 l-1} \rightarrow \mathbb{R}^{3 l}$ is non-trivial although $\alpha(w)=\Sigma^{\infty} \lambda_{12}(w)=0$.

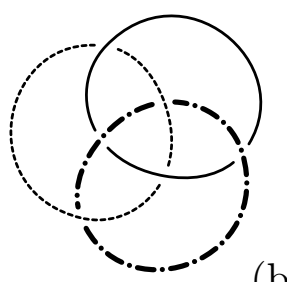

(b)

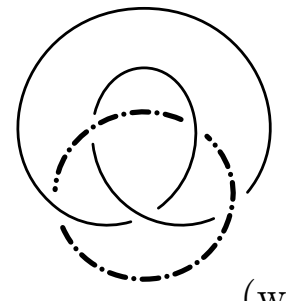

(w)

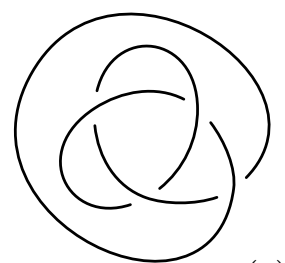

(t)

Figure 3.6

The Whitehead link is obtained from Borromean rings by joining two components with a tube (Figure 3.6.w). We have

$$
\alpha(w)=0 \quad \text { but } \quad \lambda_{12}(w)=\left[\iota_{l}, \iota_{l}\right] \neq 0 \quad \text { for } \quad l \neq 1,3,7 .
$$

Cf. [Ha62', §3]. Note that for $l=1,3,7$ the Whitehead link is still non-trivial, although $\lambda_{12}(w)=\lambda_{21}(w)=0[\mathrm{Ha} 62, \xi 3]$.

The Trefoil Knot Example 3.4. The trefoil knot $S^{2 l-1} \rightarrow \mathbb{R}^{3 l}$ is not smoothly trivial (but is PL trivial for $l \geq 2$ ) [Ha62, Ha66'].

The trefoil knot is obtained by joining the three Borromean rings by two tubes (Figure 3.6.t).

If we take a cone or a suspension over any codimension 2 knot, then we obtain a PL embedding $f$ of a ball or of a sphere which is not smoothable, i.e. is not PL isotopic to a smooth (not necessarily standard) embedding. This is so because $f$ is not locally flat. Recall that an embedding $N \subset \mathbb{R}^{m}$ of a PL $n$-manifold $N$ is locally flat if each point $x \in N$ has a closed neighborhood $U$ such that $(U, U \cap N) \cong\left(D^{m}, D^{n}\right)$. Observe that for $m \geq n+3$ the suspension extension $S^{n} \rightarrow \mathbb{R}^{m}$ of any knot $S^{n-1} \rightarrow \mathbb{R}^{m-1}$ is PL isotopic to the standard embedding and is therefore smoothable.

The Haefliger Torus Example 3.5. There is a PL embedding $S^{2 k} \times S^{2 k} \rightarrow \mathbb{R}^{6 k+1}$ which is (locally flat but) not PL isotopic to a smooth embedding [Ha62, BH70, p.165, Bo71, 6.2].

In order to construct the Haefliger torus take the above trefoil knot $S^{4 k-1} \rightarrow \mathbb{R}^{6 k}$. Extend this knot to a conical embedding $D^{4 k} \rightarrow \mathbb{R}_{-}^{6 k+1}$. By [Ha62], the trefoil knot also extends to a smooth embedding $S^{2 k} \times S^{2 k}-\stackrel{\circ}{D}^{4 k} \rightarrow \mathbb{R}_{+}^{6 k+1}$ (Figure 3.7.a). These two extensions together form the Haefliger torus (Figure 3.7.b). 


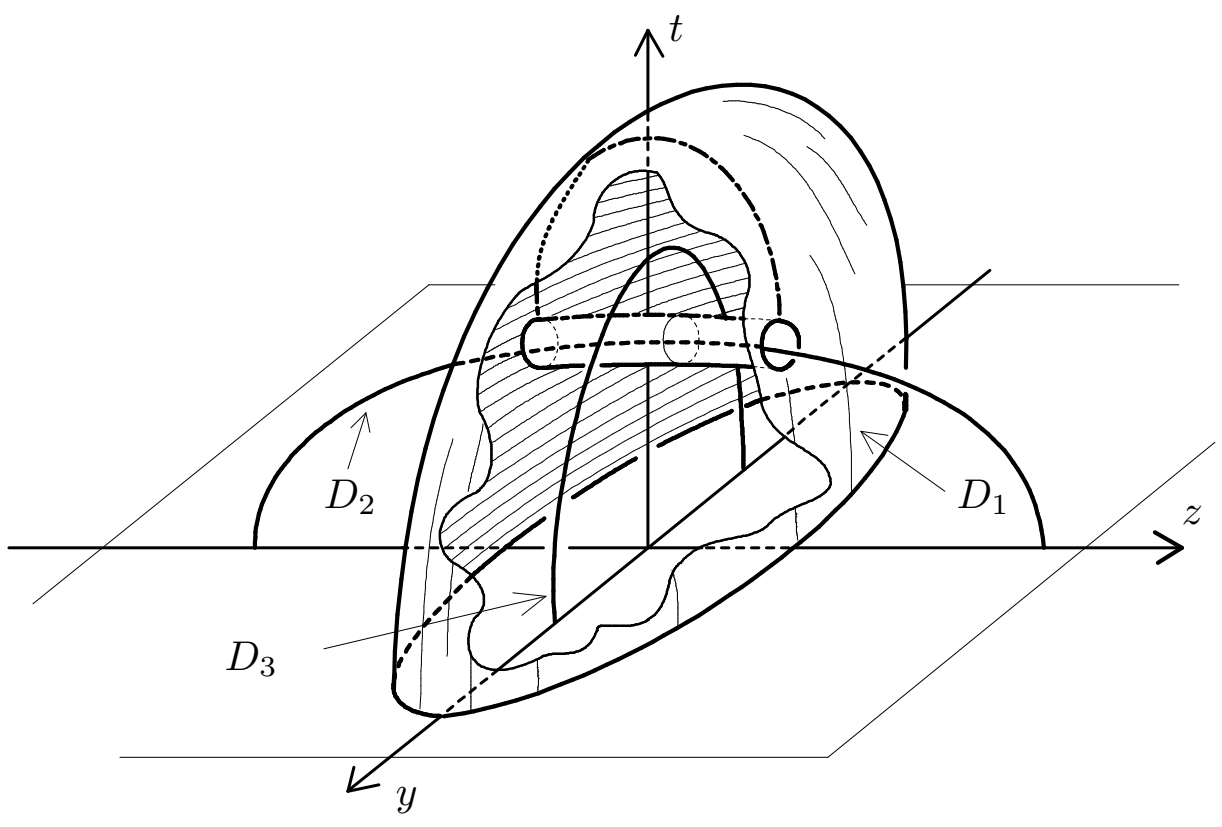

Figure 3.7.a

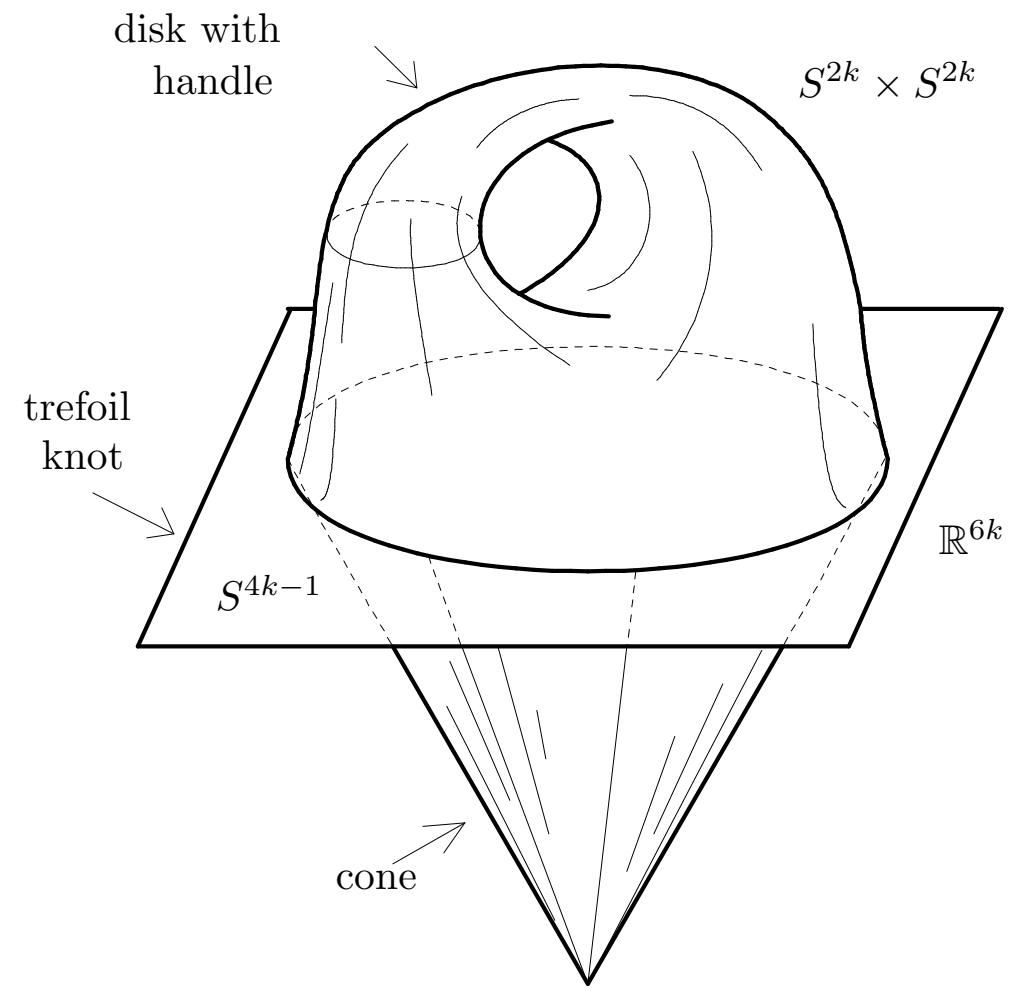

Figure 3.7.6

A classification of knots and links below the metastable range. Denote

$$
C_{q}^{m-q}:=\operatorname{Emb}_{D I F F}^{m}\left(S^{q}\right) .
$$

The 'connected sum' commutative group structure on $C_{q}^{m-q}$ was defined for $m \geq q+3$ in [Ha66, cf. Ha66']. Theorem 2.5.b states that $C_{q}^{m-q}=0$ for $2 m \geq 3 q+4$. It is known [Ha66, Mi72', KS05] that

$$
C_{4 k-1}^{2 k+1} \cong \mathbb{Z}, \quad C_{4 k+1}^{2 k+2} \cong \mathbb{Z}_{2}, \quad C_{4}^{3} \cong \mathbb{Z}_{12}, \quad C_{4 k-2}^{2 k}=0,
$$




$$
C_{8 s+4}^{4 s+3} \cong \mathbb{Z}_{4} \quad \text { for } \quad s>0 \quad \text { and } \quad C_{8 s}^{4 s+1} \cong \mathbb{Z}_{2} \oplus \mathbb{Z}_{2}
$$

Theorem 3.6. (a) [Ha66', cf. Ha86] If $p, q \leq m-3$, then

$$
\operatorname{Emb}_{D I F F}^{m}\left(S^{p} \sqcup S^{q}\right) \cong \operatorname{Emb}_{P L}^{m}\left(S^{p} \sqcup S^{q}\right) \oplus C_{q}^{m-q} \oplus C_{p}^{m-p}
$$

(b) [Ha66', Theorem 10.7, Sk'] If $p \leq q \leq m-3$ and $3 m \geq 2 p+2 q+6$, then for large enough $M$

$$
\operatorname{Emb}_{P L}^{m}\left(S^{p} \sqcup S^{q}\right) \cong \pi_{p}\left(S^{m-q-1}\right) \oplus \pi_{p+q+2-m}\left(V_{M+m-p-1, M}\right)
$$

The isomorphism in Theorem 3.6.b is given by the sum of $\lambda_{12}$-invariant and the $\beta$ invariant [Sk'].

By Theorem 3.6.b (and its proof) the invariant $\lambda_{12} \oplus \lambda_{21}$ is injective for $l \geq 2$ and PL embeddings $S^{2 l-1} \sqcup S^{2 l-1} \rightarrow \mathbb{R}^{3 l}$; its range is isomorphic to $\pi_{2 l-1}\left(S^{l}\right) \oplus \mathbb{Z}_{(l)}$. However, this invariant is not injective in other dimensions.

The set $\operatorname{Emb}^{m}\left(S^{n_{1}} \sqcup \cdots \sqcup S^{n_{s}}\right)$ for $m \geq n_{i}+3$ has been described in terms of exact sequences involving homotopy groups of spheres [Ha66, Ha66', cf. Le65, Ha86].

Knotted tori. The classification of knotted tori, i.e. the description of the isotopy classes of embeddings $S^{p} \times S^{q} \rightarrow \mathbb{R}^{m}$ is an interesting problem because

(1) it has already provided many interesting examples [Al24, Ko62, Hu63, Wa65', Ti69, BH70, Bo71, MR71, Sk02, Sk],

(2) by the Handle Decomposition Theorem it may be considered as a natural next step (after the link theory) towards the classification of embeddings for arbitrary manifolds, cf. [Sk05].

(3) it generalizes the classical theory of 2-componented links of the same dimension,

(4) it reveals new interesting relations between algebraic and geometric topology,

Denote

$$
K T_{p, q, C A T}^{m}:=\operatorname{Emb}_{C A T}^{m}\left(S^{p} \times S^{q}\right)
$$

Notice the change in the role of $p$ in this subsection compared with the previous ones. We omit CAT if a formula holds for both categories.

From the Haefliger-Zeeman Isotopy Theorem 2.6.b it follows that $K T_{p, q}^{m}=0$ for $p \leq q$ and $m \geq p+2 q+2$. The dimension restriction in this result is sharp by the Hudson Torus Example 2.6.c.

Group Structure Theorem 3.7. The set $K T_{p, q}^{m}$ has a commutative group structure for $m \geq 2 p+q+3$ in the smooth case and $m \geq \max \{2 p+q+2, q+3\}$ in the PL case [Sk].

Idea of the proof. See Figure 3.8. By [Sk] under the dimension assumptions for any embedding $f: S^{p} \times S^{q} \rightarrow \mathbb{R}^{m}$ there is a web, i.e. an embedding

$$
u: D^{p+1} \rightarrow \mathbb{R}^{m} \quad \text { such that } u\left(D^{p+1}\right) \cap f\left(S^{p} \times S^{q}\right)=u\left(\partial D^{p+1}\right)=f\left(S^{p} \times 1\right) .
$$

Moreover, a web is unique up to isotopy. 
Now take two embeddings $f_{0}, f_{1}: S^{p} \times S^{q} \rightarrow \mathbb{R}^{m}$ and their webs $D_{0}^{p+1}$ and $D_{1}^{p+1}$. Join the centers of $D_{0}^{p+1}$ and $D_{1}^{p+1}$ by an arc. Construct an embedding $\partial D^{p+1} \times I \rightarrow \mathbb{R}^{m}$ 'along this arc' so that

$$
\partial D^{p+1} \times I \cap f_{i}\left(S^{p} \times S^{q}\right)=\partial D^{p+1} \times i=f_{i}\left(S^{p} \times 1\right) \quad \text { for } \quad i=0,1 .
$$

Take a 'connected sum' of $f_{0}$ and $f_{1}$ 'along $\partial D^{p+1} \times I$ '. The resulting embedding $S^{p} \times S^{q} \rightarrow$ $\mathbb{R}^{m}$ is the sum of $f_{0}$ and $f_{1}$.

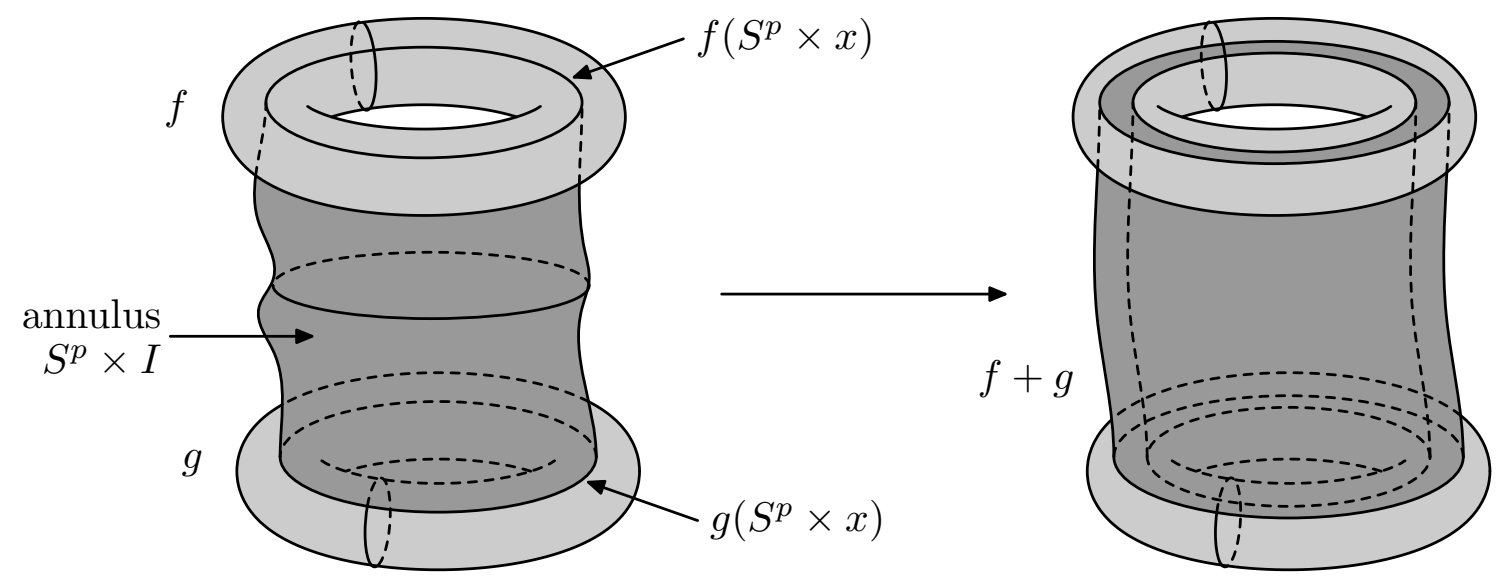

Figure 3.8

Theorem 3.8. [HH63, Hu63, Vr77]

$K T_{p, q, P L}^{p+2 q+1} \cong\left\{\begin{array}{ll}\mathbb{Z}_{(q)} & 1 \leq p<q \\ \mathbb{Z}_{(q)} \oplus \mathbb{Z}_{(q)} & 2 \leq p=q\end{array} \quad\right.$ and $\quad K T_{p, q, D I F F}^{p+2 q+1} \cong \mathbb{Z}_{(q)} \quad$ for $\quad 1 \leq p \leq q-2$.

Theorem 3.8 follows from Theorem 2.8.b (as well as from Theorem 3.9 below). In the PL case of Theorem 3.8 for $p=q$ we only have a 1-1 correspondence of sets (because Group Structure Theorem 3.7 does not give a group structure for such dimensions). A description of $K T_{2 k-1,2 k, D I F F}^{6 k}$ is given after Theorem 2.9.

This result can be generalized as follows.

Theorem 3.9. [Sk02, Corollary 1.5.a] If $2 m \geq 3 q+2 p+4$ or $2 m \geq 3 q+3 p+4$, in the PL or DIFF cases respectively, then

$$
K T_{p, q}^{m} \cong \pi_{q}\left(V_{m-q, p+1}\right) \oplus \pi_{p}\left(V_{m-p, q+1}\right) .
$$

Note that $\pi_{p}\left(V_{m-p, q+1}\right)=0$ for $m \geq 2 p+q+2$ (which is automatic for $p \leq q$ and $2 m \geq 3 p+3 q+4)$. Theorem 3.9 follows from Theorems 5.4, 5.5 and Deleted Product Lemma 5.3.b below. For $m \geq 2 p+q+2$ there is an alternative direct proof [Sk], but for $m<2 p+q+2$ (when no group structure exists) no proof of Theorem 3.9 without referring to the deleted product method is known.

Let us construct a map $\tau: \pi_{q}\left(V_{m-q, p+1}\right) \rightarrow K T_{p, q}^{m}$ giving one summand in Theorem 3.9. Recall that $\pi_{q}\left(V_{m-q, p+1}\right)$ is isomorphic to a group of CAT maps $S^{q} \rightarrow V_{m-q, p+1}$ up to CAT homotopy. The latter maps can be considered as CAT maps $\varphi: S^{q} \times S^{p} \rightarrow \partial D^{m-q}$. Define the CAT embedding $\tau(\varphi)$ (Figure 3.9) as the composition

$$
S^{p} \times S^{q} \stackrel{\varphi \times \operatorname{pr}_{2}}{\rightarrow} \partial D^{m-q} \times S^{q} \subset D^{m-q} \times S^{q} \subset \mathbb{R}^{m} .
$$




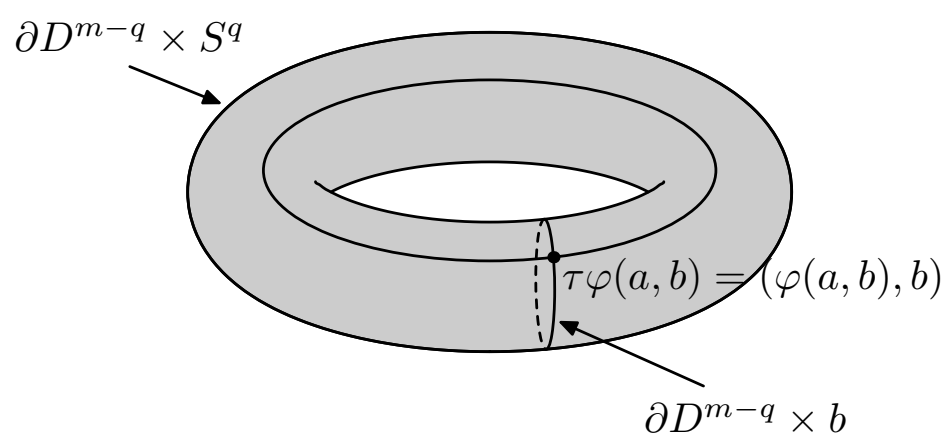

Figure 3.9

Let us present some calculations based on Theorem 3.9 and the calculation of $\pi_{q}\left(V_{a, b}\right)$ [Pa56, DP97]. Recall that $\pi_{q}\left(V_{m-q, 2}\right) \cong \pi_{q}\left(S^{m-q-1}\right) \oplus \pi_{q}\left(S^{m-q-2}\right)$ for $m-q$ even $(\cong$ $\pi_{2 q+1-m}^{S} \oplus \pi_{2 q+2-m}^{S}$ for $m-q$ even and $\left.2 m \geq 3 q+6\right)$ because the sphere $S^{m-q-1}$ has a non-zero vector field. In all tables of this subsection $u^{v}$ means $\left(\mathbb{Z}_{u}\right)^{v}$.

The Haefliger-Zeeman Theorem 3.1 suggests the following question: how can we describe $K T_{1, q}^{m}$ ? We have the following table for $2 m \geq 3 q+6$ and for $2 m \geq 3 q+7$, in the $\mathrm{PL}$ and DIFF cases respectively.

$$
\begin{aligned}
& m \quad: 2 q+2: 2 q+1: 2 q: 2 q-1: 2 q-2: 2 q-3 \\
& K T_{1, q}^{m}, q \text { even }: \mathbb{Z}: 2: 2^{2}: 2^{2}: 24: 0 \\
& K T_{1, q}^{m}, q \text { odd }: \quad 2: \mathbb{Z} \oplus 2: 4: 2 \oplus 24: \quad 2: 0
\end{aligned}
$$

Theorem 3.8 and [MR71] suggest the following problem: describe $K T_{p, q}^{m}$ for $m \leq 2 q+p$. We have the following table for $q \geq 4$ or $q \geq p+4$, in the PL or DIFF cases respectively .

$$
\begin{aligned}
& p \quad: \quad 1: 2 \leq p \leq q-2: q-1: q \\
& K T_{p, q}^{p+2 q}, q=4 s \quad: \quad 2 \quad: \quad 0 \quad: \quad 2 \quad: 0 \\
& K T_{p, q}^{p+2 q}, q=4 s+2: 2 \quad 2 \quad 2 \quad: \quad 2^{2}: 2^{2} \\
& K T_{p, q}^{p+2 q}, q=4 s+1: \mathbb{Z} \oplus 2: \quad 2^{2} \quad: \mathbb{Z} \oplus 2^{2}: 2^{4} \\
& K T_{p, q}^{p+2 q}, q=4 s-1: \mathbb{Z} \oplus 2: \quad 4 \quad: \mathbb{Z} \oplus 4: 4^{2}
\end{aligned}
$$

Classifications of smooth embeddings $S^{p} \times S^{q} \rightarrow \mathbb{R}^{m}$ for $2 m \leq 3 p+3 q+3$, as well as PL embeddings for $2 m \leq 2 p+3 q+3$ is much harder (because of the existence of smooth knots and the incompleteness of the Haefliger-Wu invariant). However, the statements are simple.

Theorem 3.10. (a) [Sk] If $p \leq q, m \geq 2 p+q+3$ and $2 m \geq 3 q+2 p+4$, then

$$
K T_{p, q, D I F F}^{m} \cong K T_{p, q}^{m} \oplus G_{m, p, q} \cong \pi_{q}\left(V_{m-q, p+1}\right) \oplus G_{m, p, q}
$$

where $G_{m, p, q}$ is certain quotient of $C_{p+q}^{m-p-q}$.

(b) [Sk06] If $1 \leq p \leq 2 k-2$, then

$$
K T_{p, 4 k-1-p}^{6 k} \cong \pi_{4 k-1-p}\left(V_{2 k+p+1, p+1}\right) \oplus \mathbb{Z} .
$$


Theorem 3.11. [Sk] (aDIFF) For each $k>1$ we have

$$
K T_{1,4 k-1, D I F F}^{6 k+1} \cong \pi_{2 k-2}^{S} \oplus \pi_{2 k-1}^{S} \oplus \mathbb{Z} \oplus G_{k},
$$

where $G_{k}$ is an abelian group of order 1, 2 or 4 .

$$
(a P L) \quad K T_{1,4 k-1, P L}^{6 k+1} \cong \pi_{2 k-2}^{S} \oplus \pi_{2 k-1}^{S} \oplus \mathbb{Z} .
$$

(b) For each $k>0$ we have

$K T_{1,4 k+1, P L}^{6 k+4} \cong K T_{1,4 k+1, D I F F}^{6 k+4} \cong \mathbb{Z}_{2}^{a} \oplus \mathbb{Z}_{4}^{b} \quad$ for some integers $a=a(k), b=b(k)$ such that

$$
a+2 b-\operatorname{rk}\left(\pi_{2 k}^{S} \otimes \mathbb{Z}_{2}\right)-\operatorname{rk}\left(\pi_{2 k-1}^{S} \otimes \mathbb{Z}_{2}\right)= \begin{cases}0 & k \in\{1,3\} \\ 1 & k+1 \text { is not a power of } 2 . \\ 1 \text { or } 0 & k+1 \geq 8 \text { is a power of } 2\end{cases}
$$

For a generalization of Theorem 3.11 and its relation to homotopy groups of Stiefel manifolds see [Sk, MS04]. By Theorem 3.11 and [Pa56, To62, DP97] (see the details in $[\mathrm{Sk}]$ ) we have the following table.

$$
\begin{gathered}
l=2=3=4 \quad=5=6=7=0 \quad 8=9=10 \\
K T_{1,2 l-1, P L}^{3 l+1}=\mathbb{Z}^{2} \oplus 2=4=\mathbb{Z} \oplus 24 \oplus 2=2^{2}=\mathbb{Z}=2=\mathbb{Z} \oplus 240 \oplus 2=2 \times 22^{2}=\mathbb{Z} \oplus 2^{5}
\end{gathered}
$$

The following strong result was proved using a clever generalization of methods from $[\mathrm{Sk}]$.

Theorem 3.12. Assume that

$$
p \leq q, \quad p+\frac{4}{3} q+2<m<p+\frac{3}{2} q+2 \quad \text { and } \quad m>2 p+q+2 .
$$

The group $K T_{p, q, D I F F}^{m}$ is infinite if and only if either $q+1$ or $p+q+1$ is divisible by 4 [CRS].

We conclude this subsection by some open problems. It would be interesting to find $\widehat{C_{p+q}^{m-p-q}}$, at least for particular cases. It would be interesting to describe $K T_{2 k, 2 k+1, D I F F}^{6 k+2}$. Note that $\# K T_{2 k, 2 k+1, D I F F}^{6 k+2} \in\{2,3,4\}$ [Sk06], cf. Theorem 3.9.b. It would be interesting to find $K T_{2 k, 2 k+1, D I F F}^{6 k+3}$. For this case the Whitney invariant is a surjection onto $\mathbb{Z}_{2}$, and both preimages consist on 1 or 2 elements. It would be interesting to find $K T_{q, q, D I F F}^{3 q+1}$ for $q \geq 2$. For this case the image of the Whitney invariant is $\mathbb{Z} \vee \mathbb{Z}$ for $q$ even and is either $\mathbb{Z}_{2} \vee \mathbb{Z}_{2}$ or $\mathbb{Z}_{2} \oplus \mathbb{Z}_{2}$ for $q$ odd. For a group $G$ define $G \vee G=\{(x, y) \in G \oplus$ $G \mid$ either $x=0$ or $y=0\}$. The non-empty preimages of the Whitney invariant consist of 1 element for $q$ odd, of 1,2,4 elements for $q$ even $\geq 4$, and of 1,2,3,4,6,12 elements for $q=2$.

It would be interesting to find an action of the group of CAT auto-homeomorphisms of $S^{p} \times S^{q}$ on $K T_{p, q, C A T}^{m}$ (E. Rees). The above classification of knotted tori could perhaps be applied to solve for knotted tori the Hirsch problem about the description of possible normal bundles for embeddings of manifolds into $\mathbb{R}^{m}$, cf. [MR71]. The same remark holds for the following Hirsch-Rourke-Sanderson problem [Hi66, RS01, cf. Ti69, Vr89]: which embeddings $N \rightarrow \mathbb{R}^{m+1}$ are isotopic to embeddings $N \rightarrow \mathbb{R}^{m}$ ? 


\section{The VAn Kampen OBStruCtion}

The embeddability of $n$-complexes in $\mathbb{R}^{2 n}$. By the General Position Theorem 2.1.a, the first non-trivial case of the Embedding Problem is the investigation of the embeddability of $n$-polyhedra in the Euclidean space $\mathbb{R}^{2 n}$, cf. Example 2.1.b. For $n=1$ this problem was solved by the Kuratowski criterion [Ku30], see also [RS96, §2, Sk05'] and references there. However for $n>1$, such a simple criterion does not exist [Sa91]. (Note that there are infinitely many closed non-orientable 2-surfaces, which do not embed into $\mathbb{R}^{3}$ and these do not contain a common subspace non-embeddable into $\mathbb{R}^{3}$.) In [Ka32] an obstruction to the embeddability of $n$-polyhedra in $\mathbb{R}^{2 n}$ was constructed for arbitrary $n$ (see also a historical remark at the end of $\S 5$ ).

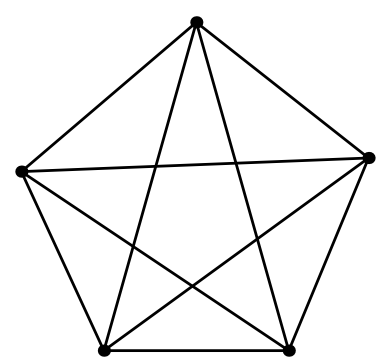

Figure 4.1

To explain the idea of van Kampen, we sketch a proof of the nonplanarity of $K_{5}$ (i.e. of the complete graph with 5 vertices, Figure 4.1). Take any general position map $f: K_{5} \rightarrow \mathbb{R}^{2}$. For each two edges $\sigma, \tau$ the intersection $f \sigma \cap f \tau$ consists of a finite number of points. Let $v_{f}$ be

the sum mod 2 of the numbers $|f \sigma \cap f \tau|$ over all non-ordered pairs $\{\sigma, \tau\}$ of disjoint edges of $K_{5}$.

For the map $f$, shown in Figure 4.1, $v_{f}=1$. Every general position map $f: K_{5} \rightarrow \mathbb{R}^{2}$ can be transformed to any other such map through isotopies of $\mathbb{R}^{2}$ and 'the Reidemeister moves' for graphs in the plane from Figure 4.2.
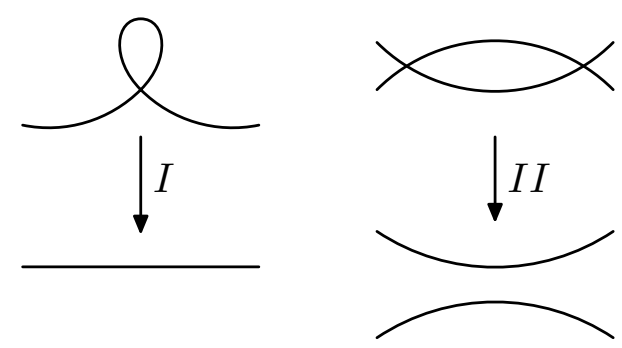

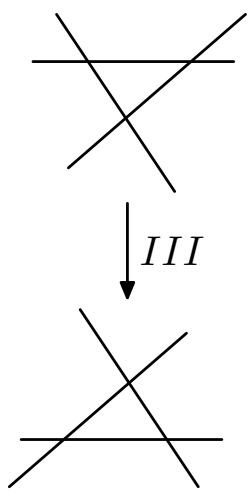

Figure 4.2
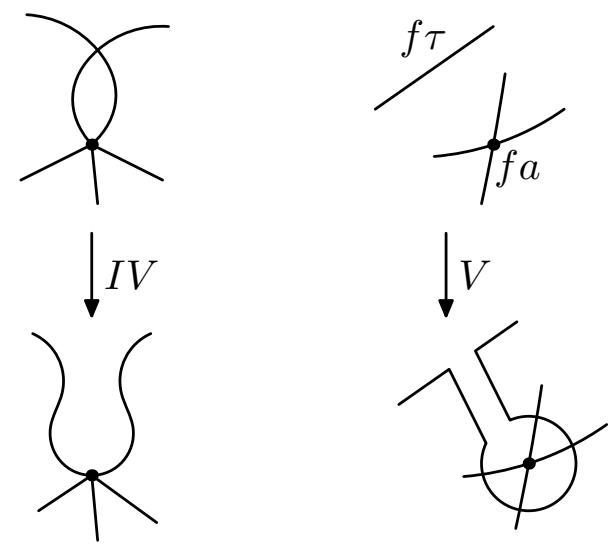

This assertion is proved analogously to the Reidemeister theorem for knots. We will not prove it, since it is needed only for this sketch proof and not for the rigorous proof. For each edge of $K_{5}$ with vertices $a, b$, the graph $K_{5}-\{a, b\}$, obtained by deleting from $K_{5}$ 
the vertices $a, b$ and the interiors of the edges adjacent to $a, b$, is a circle (this is the very property of $K_{5}$ we need for the proof). Therefore $v_{f}$ is invariant under the 'Reidemeister moves'. Hence $v_{f}=1$ for each general position map $f: K_{5} \rightarrow \mathbb{R}^{2}$. So $K_{5}$ is nonplanar. (For a proof without use of the assertion on the Reidemeister moves see below or [Sk05"].)

Similarly, one can prove that the graph $K_{3,3}$ (three houses and three wells) is not embeddable into $\mathbb{R}^{2}$ and that the 2-skeleton of the 6 -simplex is not embeddable into $\mathbb{R}^{4}$, cf. Example 2.1.b.

Ramsay link theory. Now let us discuss some generalizations of the above proof which are interesting in themselves and are used in $\S 7$. From that proof one actually gets a stronger assertion. Let $e$ be an edge of $K_{5}$ and $\Sigma^{1}$ the cycle in $K_{5}$, formed by the edges of $K_{5}$ disjoint with $e$. Then $K_{5}-\check{e}$ embeds into $\mathbb{R}^{2}$ (Figure 4.3) and for each embedding

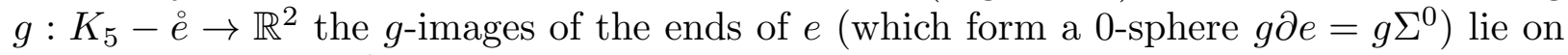
different sides of $g \Sigma^{1}$.

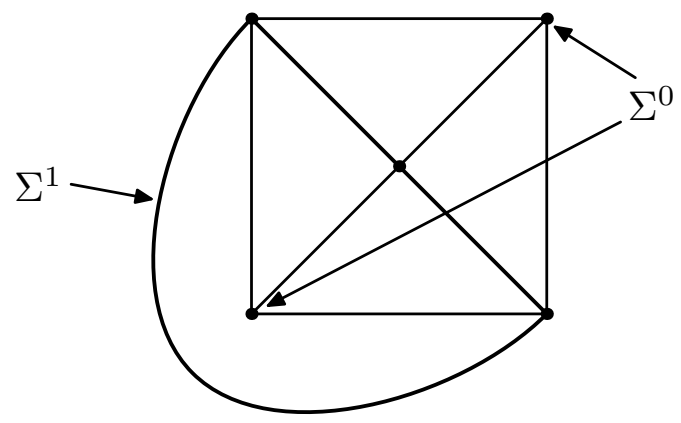

Figure 4.3

Moreover, let $e$ be a 2 -simplex of the 2-skeleton $\Delta_{6}^{2}$ of the 6-simplex and $P=\Delta_{6}^{2}-\stackrel{e}{\text {. }}$ Then $P$ embeds into $\mathbb{R}^{4}$. Let $\Sigma^{1}=\partial e$ and $\Sigma^{2}$ be the sphere formed by 2 -faces disjoint with $e$. Then for each embedding $P \rightarrow \mathbb{R}^{4}$ the images of these spheres link with a non-zero (more precisely, with an odd) linking number [Fl34].

Using the above idea one can prove the following.

For any embedding $K_{6} \rightarrow \mathbb{R}^{3}$ there are two cycles in $K_{6}$ whose images are linked with an odd linking coefficient [Sa81, CG83, for generalizations see RST95, Ne98, BKK02, Sk03, PS05].

The van Kampen obstruction mod 2. Fix a triangulation $T$ of a polyhedron $N$. The space

$$
\widetilde{T}=\cup\{\sigma \times \tau \in T \times T \mid \sigma \cap \tau=\emptyset\}
$$

is called the simplicial deleted product of $N$. By the Simplicial Deleted Product Lemma 5.3. a below the equivariant homotopy type of $\widetilde{T}$ depends only on $N$, so we write $\widetilde{N}$ instead of $\widetilde{T}$ in this section. Consider the 'exchanging factors' involution $t: \widetilde{N} \rightarrow \widetilde{N}$ defined by $t(x, y)=(y, x)$. Let $N^{*}=\widetilde{N} / t$.

Definition of the mod 2 van Kampen obstruction $v(N)$ for $n=\operatorname{dim} N=1$. For any general position PL map $f: N \rightarrow \mathbb{R}^{2}$ and disjoint edges $\sigma, \tau$ of $T$, the intersection $f(\sigma) \cap f(\tau)$ consists of a finite number of points. Let

$$
v_{f}(\sigma, \tau)=|f(\sigma) \cap f(\tau)| \bmod 2 .
$$


Then $v_{f}$ is an element of the group $C^{2}\left(N^{*} ; \mathbb{Z}_{2}\right)$ of vectors (which are called cochains) with components from $\mathbb{Z}_{2}$ indexed by 2 -simplices of $N^{*}$, i.e. by non-ordered products of disjoint edges of $N$.

This cochain $v_{f}$ is invariant under isotopy of $\mathbb{R}^{2}$ and the first four 'Reidemeister moves' (Figure 4.2.I-IV). The fifth 'Reidemeister move' (passing of $f \tau$ through $f a$, see Figure 4.2.V) adds to $v_{f}$ the vector that assumes value 1 on the class of 2-simplex $\sigma \times \tau$ for $a \in \sigma$, and value 0 on the other 2 -simplices of $N^{*}$. This vector is denoted by $\delta[a \times \tau]$. Denote by $B^{2}\left(N^{*} ; \mathbb{Z}_{2}\right)$ the subgroup of $C^{2}\left(N^{*} ; \mathbb{Z}_{2}\right)$ generated by elementary coboundaries. The van Kampen obstruction mod2 is the equivalence class

$$
v(N):=\left[v_{f}\right] \in H^{2}\left(N^{*} ; \mathbb{Z}_{2}\right)=C^{2}\left(N^{*} ; \mathbb{Z}_{2}\right) / B^{2}\left(N^{*} ; \mathbb{Z}_{2}\right) .
$$

(Since $\operatorname{dim} N^{*}=2$, it follows that $C^{2}=Z^{2}$.)

Sketch of a proof that $v(N)$ is indeed independent of $f$ (without using the assertion about 'the Reidemeister moves' which is not proved here). We follow [Sh57, Lemma 3.5]. Consider an arbitrary general position homotopy $F: N \times I \rightarrow \mathbb{R}^{2} \times I$ between general position maps $f_{0}, f_{1}: N \rightarrow \mathbb{R}^{2}$. Colour a non-ordered pair $a \times \tau$ in red if $F(a \times I)$ intersects $F(\tau \times I)$ in an odd number of points. Then $v_{f_{0}}-v_{f_{1}}$ is the sum of $\sum \delta(a \times \tau)$ over all red pairs $a \times \tau$. Hence $v_{f}$ is independent on $f$.

Clearly, $v(N)=0$ for all planar graphs $N$.

Analogously one defines the mod 2 van Kampen obstruction $v(N) \in H^{2 n}\left(N^{*} ; \mathbb{Z}_{2}\right)$ to the embeddability of an $n$-polyhedron $N$ into $\mathbb{R}^{2 n}$.

The integral van Kampen obstruction. Fix a triangulation of $N$ and define $\widetilde{N}, t$ and $N^{*}$ as above. Choose an orientation of $\mathbb{R}^{2 n}$ and orientations of the $n$-simplices of $\widetilde{N}$. The latter give orientations on $2 n$-simplices of $\widetilde{N}$. Clearly, $t(\sigma \times \tau)=(-1)^{n}(\tau \times \sigma)$ (the case $n=1$ helps to check the sign) [Sh57, p.257, above].

For any general position map $f: N \rightarrow \mathbb{R}^{2 n}$ and any two disjoint $n$-simplices $\sigma, \tau$ of $N$ the intersection $f(\sigma) \cap f(\tau)$ consists of a finite number of points. Define the the intersection cochain

$$
V_{f} \in C^{2 n}(\tilde{N}) \quad \text { by the formula } \quad V_{f}(\sigma, \tau)=f \sigma \cdot f \tau:=\sum_{P \in f \sigma \cap f \tau} \operatorname{sign} P .
$$

Here sign $P=+1$, if for the positive $n$-bases $s_{1}, \ldots, s_{n}$ and $t_{1}, \ldots, t_{n}$ of $\sigma$ and $\tau$, respectively, we have that $f s_{1}, \ldots, f s_{n}, f t_{1}, \ldots, f t_{n}$ is a positive $2 n$-base of $\mathbb{R}^{2 n}$; and $\operatorname{sign} P=-1$ otherwise. Clearly [Sh57],

$$
V_{f}(\sigma \times \tau)=(-1)^{n} V_{f}(\tau \times \sigma)=V_{f}(t(\sigma \times \tau)) .
$$

So $V_{f}$ induces a cochain in the group $C^{2 n}\left(N^{*}\right)$. Denote this new cochain by the same notation $V_{f}$ (we use the old $V_{f}$ only in the proof of Lemma 4.2, so no confusion will arise). The van Kampen obstruction is the equivalence class

$$
V(N):=\left[V_{f}\right] \in H^{2 n}\left(N^{*}\right)=C^{2 n}\left(N^{*}\right) / B^{2 n}\left(N^{*}\right) .
$$

This class is independent on $f$ (this is proved analogously to the proof for $v(N)$ ). Clearly, $V(N)$ is an obstruction to the embeddabiltiy of $N$ into $\mathbb{R}^{2 n}$. 
One can easily show that $V(N)$ depends on the choice of orientations of $\mathbb{R}^{2 n}$ and of the $n$-simplices of $N$ only up to an automorphism of the group $H^{2 n}\left(N^{*}\right)$.

(The author is grateful to S. Melikhov for indicating that in [FKT94, Kr00, BKK02] the signs are not accurate and so the Van Kampen obstruction for $n$ odd erroneously assumes its values in in $H_{\mathbb{Z}_{2}}^{2 n}(\widetilde{N} ; \mathbb{Z})$, where the involution acts on $\widetilde{N}$ by exchanging factors and on $\mathbb{Z}$ by $(-1)^{n}$.)

\section{The van Kampen-Shapiro-Wu Theorem.}

Theorem 4.1. If an n-polyhedron $N$ embeds into $\mathbb{R}^{2 n}$, then $V(N)=0$. For $n \neq 2$ the converse is true, whereas for $n=2$ it is not [Ka32, Sh57, Wu58, FKT94].

The necessity of $V(N)=0$ in Theorem 4.1 was actually proved in the construction of the van Kampen obstruction. The sufficiency in Theorem 4.1 for $n \geq 3$ follows from Lemmas 4.2 and 4.3 below, and for $n=1$ is obtained using the Kuratowski graph planarity criterion. A counterexample to the completeness of the van Kampen obstruction for $n=2$ is presented in $\S 7$.

A map $g: N \rightarrow \mathbb{R}^{m}$ is of a polyhedron $N$ is called a non-degenerate almost embedding if there exists a triangulation $T$ of $N$ such that $\left.g\right|_{\alpha}$ is an embedding for each $\alpha \in T$ and $g \alpha \cap g \beta=\emptyset$ for each $\alpha \times \beta \subset \widetilde{T}$.

Lemma 4.2. If $N$ is an n-polyhedron and $V(N)=0$, then there exists a general position non-degenerate almost embedding $g: N \rightarrow \mathbb{R}^{2 n}$ [cf. FKT94, Lemmas 2 and 4].

Sketch of the proof. Let $T$ be a triangulation of $N$. Let $\varphi: N \rightarrow \mathbb{R}^{2 n}$ be a map linear on the simplices of $T$. Then $\varphi$ is non-degenerate. The condition $V(N)=0$ implies that $V_{\varphi} \in$ $C^{2 n}(\tilde{N})$ is an symmetric coboundary. Hence $V_{\varphi}$ equals to the sum of some 'elementary' symmetric coboundaries $\delta\left(\sigma^{n} \times \nu^{n-1}\right)+\delta t\left(\sigma^{n} \times \nu^{n-1}\right)$ over some $\sigma^{n} \times \nu^{n-1} \in \widetilde{T}$. Applying the van Kampen finger moves (higher-dimensional analogue of Figure 4.2.V) for all pairs $\sigma^{n} \times \nu^{n-1}$ from this sum we obtain a general position nondegenerate map $f: N \rightarrow \mathbb{R}^{2 n}$ such that $f \alpha \cdot f \beta=0$ for each $\alpha, \beta \in \widetilde{T}$.

Then by induction on pairs of $n$-simplices of $\widetilde{T}$ and using the Whitney trick (see below) in the inductive step we obtain the required map $g$. See the details in [FKT94].

Let us illustrate the application of the Whitney trick by the following argument.

Sketch of the proof of Theorem 2.2.a in the smooth case, stating that every smooth $n$-manifold $N$ smoothly embeds into $\mathbb{R}^{2 n}$. For $n \leq 2$ the proof is trivial, so assume that $n \geq 3$. Using the higher-dimensional analogue of the first Reidemeister move (Figure 4.2.I), any smooth general position map $f: N \rightarrow \mathbb{R}^{2 n}$ can be modified so that a single self-intersection point with a prescribed sign will be added. Hence there exists a general position map $f: N \rightarrow \mathbb{R}^{2 n}$ whose self-intersections consist of an even number of isolated points, with algebraic sum zero.

In order to conclude the proof, we 'kill' these double points in pairs. This procedure is analogous to the second Reidemeister move (Figure 4.2.II) and is called the Whitney trick. More precisely, take two double points of opposite sign:

$$
x_{1}, y_{1}, x_{2}, y_{2} \in N \quad \text { so that } \quad f\left(x_{1}\right)=f\left(x_{2}\right), \quad f\left(y_{1}\right)=f\left(y_{2}\right)
$$

Join $x_{1}$ to $y_{1}$ and $x_{2}$ to $y_{2}$ by arcs $l_{1}$ and $l_{2}$ so that these double points have 'opposite signs' along these arcs (Figure 4.4). 


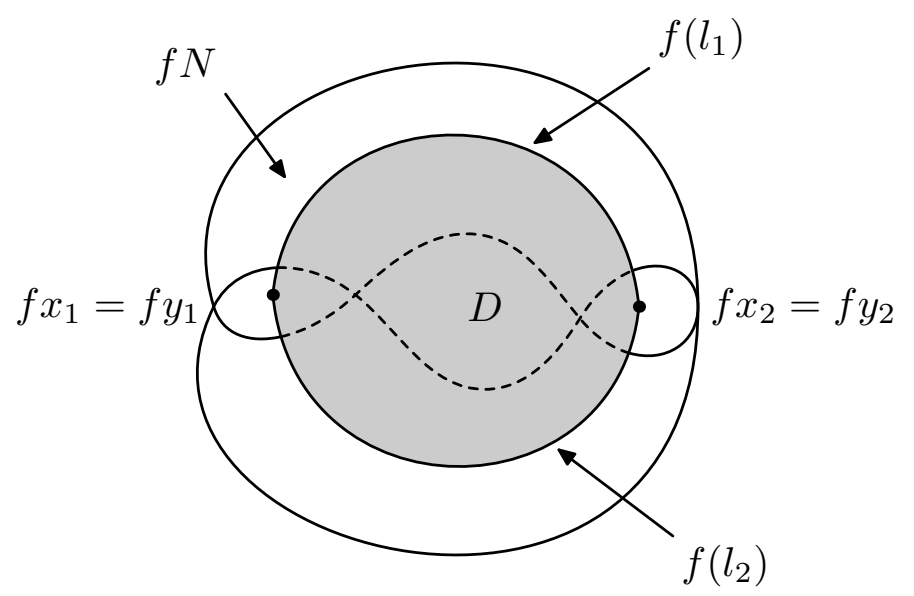

Figure 4.4

By general position $(n \geq 2)$, we may assume that the restrictions $\left.f\right|_{l_{1}}$ and $\left.f\right|_{l_{2}}$ are embeddings and that $l_{1}$ and $l_{2}$ do not contain other double points of $f$. Since $n \geq 3$, by general position we can embed a 2 -disk $C$ into $\mathbb{R}^{2 n}$ so that

$$
\partial C=f\left(l_{1}\right) \cup f\left(l_{2}\right) \quad \text { and } \quad C \cap f(N)=\partial C .
$$

Such a disk $D$ is called Whitney's disk. We can move the $f$-image of a regular neighborhood of $l_{1}$ in $N$ 'along' $C$ so that we 'cancel' the double points $f\left(x_{1}\right)=f\left(x_{2}\right)$ and $f\left(y_{1}\right)=f\left(y_{2}\right)$. For details see $[\operatorname{Ad} 93, \operatorname{Pr}]$.

The Freedman-Krushkal-Teichner Lemma 4.3. If there is a non-degenerate almost embedding $g: N \rightarrow \mathbb{R}^{2 n}$ of an n-polyhedron $N$ and $n \geq 3$, then there is an embedding $f: N \rightarrow \mathbb{R}^{2 n}$ [FKT94, Lemma 5, cf. We67, §6, Sk98].

Proof. Take a triangulation $T$ satisfying to the properties from the definition of an nondegenerate almost embedding. We may assume by induction that $g \alpha \cap g \beta=g(\alpha \cap \beta)$ for each $\alpha, \beta \in T$ such that $\alpha \cap \beta \neq \emptyset$ except for $(\alpha, \beta)=\left(\sigma^{n}, \tau^{n}\right)$. In addition, we may assume that $g \dot{\sigma}^{n} \cap g \dot{\tau}^{n}$ is a point (say, $p$ ). Let $v$ be a point of $\sigma^{n} \cap \tau^{n}$. Take PL arcs $l_{1} \subset v \cup \stackrel{\circ}{\sigma}^{p}$ and $l_{2} \subset v \cup \dot{\sim}^{q}$ joining $v$ to $g^{-1} p$ and containing no self-intersection points of $g$ in their interiors (Figure 4.5).
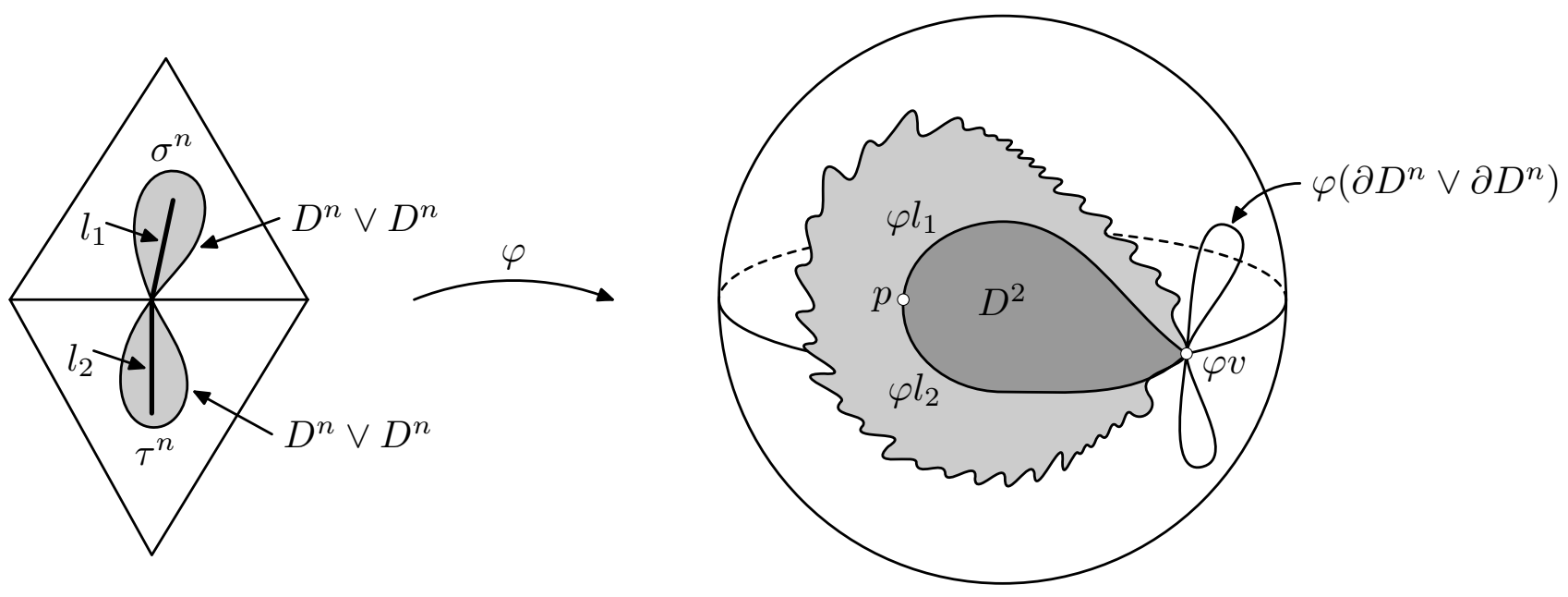

Figure 4.5 
Then $g\left(l_{1} \cup l_{2}\right)$ is a circle. Since $n \geq 3$, this circle bounds a PL embedded 2-disk

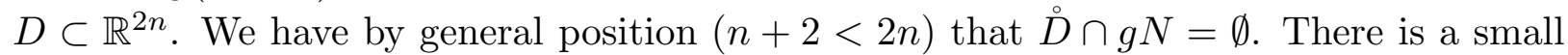
neighborhood $D^{2 n}$ of $D$ in $\mathbb{R}^{2 n}$ rel $g(v)$ that is PL homeomorphic to the $2 n$-ball and such that $g^{-1} D^{2 n}$ is a neighborhood of $l_{1} \cup l_{2}$ in $N$ rel $v$ and is homeomorphic to the wedge $D^{n} \vee D^{n}$. By the Unknotting Wedges theorem [Li65], the restriction $g: \partial g^{-1} D^{2 n} \rightarrow \partial D^{2 n}$ is unknotted. Hence it can be extended to an embedding $h: \partial g^{-1} D^{2 n} \rightarrow D^{2 n}$. In order to conclude the proof, set $f$ equal to $g$ on $N-\partial g^{-1} D^{2 n}$ and to $h$ on $\partial g^{-1} D^{2 n}$.

Observe that for the embedding $f$ constructed above we have $\widetilde{f} \simeq_{e q} \widetilde{g}$ on $\widetilde{T}$.

Generalizations of the van Kampen obstruction. The idea of the van Kampen obstruction can be applied to calculate the minimal $m$ such that a polyhedron, which is a product of graphs, embeds into $\mathbb{R}^{m}$ [Sk03, cf. Ga92, ARS01'].

Analogously, one can construct the van Kampen-Wu invariant $U(f) \in H^{2 n}\left(N^{*}\right)$ of an embedding $f: N \rightarrow \mathbb{R}^{2 n+1}$.

Theorem 4.4. If embeddings $f, g: N \rightarrow \mathbb{R}^{2 n+1}$ of a finite $n$-polyhedron $N$ are isotopic, then $U(f)=U(g)$. For $n \geq 2$ the converse is true, whereas for $n=1$ it is not [Wu65].

Note that

embeddings $f, g: N \rightarrow \mathbb{R}^{3}$ of a graph $N$ such that $U(f)=U(g)$ are homologous [Ta95].

In this paper we shall not present a proof of Theorem 4.4. For $n \geq 2$ it is proved analogously to Theorem 4.1 using the ideas of [RS99, $\S 12]$, and for $n=1$ it is trivial.

As it was pointed out by Shapiro, when $V(N)=0$ (and hence $N$ is embeddable in $\mathbb{R}^{2 n}$ for $n \geq 3$ ), one can construct the 'second obstruction' to the embeddability of $N$ in $\mathbb{R}^{2 n-1}$, etc.

For a subpolyhedron $A$ of a polyhedron $N$ one can analogously define the obstruction to extending given embedding $A \subset \partial B^{m}$ to an embedding $N \rightarrow B^{m}$ [FKT94]. The relative van Kampen obstruction is complete for $n \neq 2$ (for $n \geq 3$ see [Wu65] and for $n=1$ this follows from a relative version of the Kuratowski criterion [Sk05']) and is incomplete for $n=2(\S 7)$.

For the van Kampen obstruction for approximability by embeddings see [CRS98, §4, RS98, ARS02, §4, RS02, Me02, Sk03’].

\section{The Haefliger-Wu invariant}

Basic idea. 'The complement of the diagonal' and 'the Gauss map' ideas play a great role in different branches of mathematics [Gl68, Va92]. The Haefliger-Wu invariant is a manifestation of these ideas in the theory of embeddings. The complement to the diagonal idea originated from two celebrated theorems: the Lefschetz Fixed Point Theorem and the Borsuk-Ulam Antipodes Theorem. In order to state the latter denote the antipode of a point $x \in S^{n}$ by $-x$ and recall that a map $f: S^{n} \rightarrow S^{m}$ between spheres is equivariant (or odd), if $f(-x)=-f(x)$ for each $x \in S^{n}$.

The Borsuk-Ulam Theorem 5.1. (a) For any map $f: S^{n} \rightarrow \mathbb{R}^{n}$ there exists $x \in S^{n}$ such that $f(x)=f(-x)$.

(b) There are no equivariant maps $S^{n} \rightarrow S^{n-1}$.

(c) Every equivariant map $S^{n} \rightarrow S^{n}$ is not homotopic to the constant map.

Sketch of a deduction of (a) and (b) from (c). Part (c) is non-trivial, see the proof e.g. in $[\operatorname{Pr} 04,8.8]$. Part (b) follows from (c) because if $\varphi: S^{n} \rightarrow S^{n-1}$ is an equivariant map, then 
the restriction $\left.\varphi\right|_{S^{n-1}}$ extends to $S^{n}$ and therefore is null-homotopic. In order to present the idea of the Gauss map in the simplest case, let us deduce (a) from (b). Suppose to the contrary, that there exists a map $f: S^{n} \rightarrow \mathbb{R}^{n}$ which does not identify any antipodes. Then a map

$$
\tilde{f}: S^{n} \rightarrow S^{n-1} \quad \text { is well-defined by } \quad \tilde{f}(x)=\frac{f(x)-f(-x)}{|f(x)-f(-x)|} .
$$

Evidently, $\widetilde{f}$ is equivariant. This contradicts to (b).

Construction of Example 2.1.b. We present a simplified construction invented by Schepin and the author (and, possibly, others), cf. [Sc84, Appendix, RS01']. Let $T$ be a triod, i.e. the graph with four vertices $O, A, B, C$ and three edges $O A, O B$ and $O C$. The product $T^{n+1}$ is a cone over some $n$-polyhedron $N$.

In order to prove that $N$ does not embed into $\mathbb{R}^{2 n}$ it suffices to prove that $T^{n+1}$ does not embed in $\mathbb{R}^{2 n+1}$. Suppose to the contrary that there is an embedding $f: T^{n+1} \rightarrow \mathbb{R}^{2 n+1}$. Let $p: D^{2} \rightarrow T$ be a map which does not identify any antipodes of $S^{1}=\partial D^{2}$ (e.g. the map from Figure 5.1). It is easy to check that the map $\left.p^{n+1}\right|_{\partial D^{2 n+2}}: \partial D^{2 n+2} \rightarrow T^{n+1}$ also does not identify any antipodes. Then the composition of $p^{n+1}$ and $f$ again does not identify antipodes. This contradicts the Borsuk-Ulam Theorem 5.1.a.

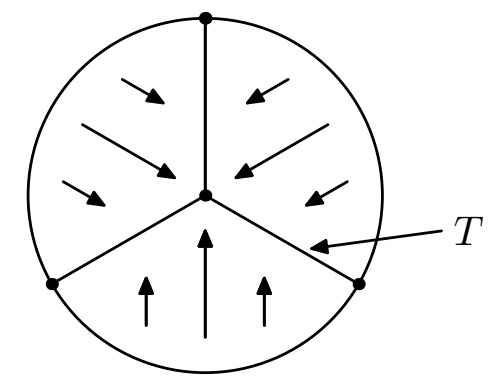

Figure 5.1

Definition of the Haefliger-Wu invariant. The deleted product $\widetilde{N}$ of a topological space $N$ is the product of $N$ with itself, minus the diagonal:

$$
\widetilde{N}=\{(x, y) \in N \times N \mid x \neq y\} .
$$

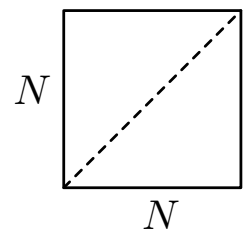

Figure 5.2

This is the configuration space of ordered pairs of distinct points of $N$.

Now suppose that $f: N \rightarrow \mathbb{R}^{m}$ is an embedding. Then the map $\widetilde{f}: \widetilde{N} \rightarrow S^{m-1}$ is well-defined by the Gauss formula

$$
\widetilde{f}(x, y)=\frac{f(x)-f(y)}{|f(x)-f(y)|} .
$$




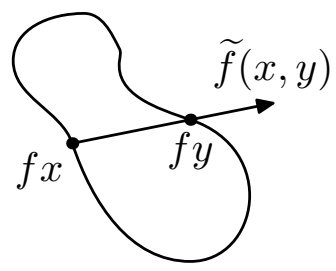

Figure 5.3

This map is equivariant with respect to the 'exchanging factors' involution $t(x, y)=$ $(y, x)$ on $N$ and the antipodal involution $a_{m-1}$ on $S^{m-1}$. Thus the existence of at least one equivariant map $\widetilde{N} \rightarrow S^{m-1}$ is a necessary condition for the embeddability of $N$ in $\mathbb{R}^{m}$.

For isotopic embeddings $f_{0}, f_{1}: N \rightarrow \mathbb{R}^{m}$ and an isotopy $f_{t}: N \times I \rightarrow \mathbb{R}^{m}$ between them the homotopy $\widetilde{f}_{t}$ is an equivariant homotopy between $\widetilde{f}_{0}$ and $\tilde{f}_{1}$. Hence the existence of an equivariant homotopy between $\widetilde{f}_{0}$ and $\widetilde{f}_{1}$ is necessary for the embeddings $f_{0}, f_{1}: N \rightarrow \mathbb{R}^{m}$ to be isotopic. The Haefliger-Wu invariant $\alpha(f)$ of the embedding $f$ is the equivariant homotopy class of the map $\widetilde{f}$, cf. [Wu59, Ha61, Gr86, 2.1.E].

Let $\pi_{e q}^{m-1}(\widetilde{N})=\left[\widetilde{N} ; S^{m-1}\right]_{e q}$ be the set of equivariant maps $\widetilde{N} \rightarrow S^{m-1}$ up to equivariant homotopy. Thus the Haefliger-Wu invariant is a mapping

$$
\alpha=\alpha_{C A T}^{m}(N): \operatorname{Emb}_{C A T}^{m}(N) \rightarrow \pi_{e q}^{m-1}(\tilde{N}) \quad \text { defined by } \quad \alpha(f)=[\tilde{f}] \in \pi_{e q}^{m-1}(\tilde{N}) .
$$

Calculations of the Haefliger-Wu invariant. It is important that using algebraic topology methods the set $\pi_{e q}^{m-1}(\widetilde{N})$ can be explicitly calculated in many cases Let us give several examples, cf. [FS59, CF60, beginning of $\S 2$, Ha62", Ha63, 1.7.1, Ba75, Ad93, 7.1, RS99, Sk02]. We denote by ' $\cong$ ' the existence of a 1-1 correspondence between sets.

The Deleted Product Lemma 5.3. (a) $\pi^{m-1}\left(\widetilde{S^{p} \sqcup S^{q}}\right) \cong \pi_{p+q+1-m}^{S}$ for $m \geq q+2$.

(b) $\pi^{m-1}\left(\widetilde{S^{p} \times S^{q}}\right) \cong \pi_{q}\left(V_{m-q, p+1}\right) \oplus \pi_{p}\left(V_{m-p, q+1}\right)$ for $2 m \geq 3 q+2 p+4$.

The Deleted Product Lemma 5.3.a and 5.3.b is proved in $\S 6$ and follows from the Torus Lemma 6.1, respectively. From the proof of the Deleted Product Lemma 5.3.a it follows that the $\alpha$-invariants of $\S 3$ and of $\S 5$ for $N=S^{p} \sqcup S^{q}$ indeed coincide.

The Simplicial Deleted Product Lemma 5.3.c. Fix a triangulation $T$ of a polyhedron N. The simplicial deleted product

$$
\widetilde{T}=\cup\{\sigma \times \tau \in T \times T \mid \sigma \cap \tau=\emptyset\}
$$

is an equivariant strong deformation retract of $\widetilde{N}$ [Sh5\%, Lemma 2.1, Hu60, §4].

Sketch of a proof. Denote

$$
E_{\sigma \tau}:=\cup\left\{U_{\sigma} \times U_{\tau} \mid U_{\sigma}, U_{\tau} \text { non-empty faces of } \sigma, \tau \text {, respectively, and } U_{\sigma} \cap U_{\tau}=\emptyset\right\} \text {. }
$$

Then $\sigma \times \tau \cong E_{\sigma \tau} * \operatorname{diag}(\sigma \cap \tau)$. So for $\sigma \cap \tau \neq \emptyset$ there is an equivariant strong deformation retraction $\sigma \times \tau-\operatorname{diag}(\sigma \cap \tau) \rightarrow E_{\sigma \tau}$. These retractions agree on intersections, so together they form an equivariant strong deformation retraction $\widetilde{N} \rightarrow \widetilde{T}$. 
Now we sketch how to deduce in a purely algebraic way all the necessary conditions for embeddability and isotopy presented in $\S 2$ and $\S 4$ from the 'deleted product necessary conditions'. Let $N$ be a polyhedron (in particular, a smooth manifold). By the Simplicial Deleted Product Lemma 5.3.c, there exists an equivariant map $\widetilde{N} \rightarrow S^{m-1}$ if and only if there exists an equivariant map $\widetilde{T} \rightarrow S^{m-1}$. Define an $S^{m-1}$-bundle

$$
\gamma: \frac{\widetilde{T} \times S^{m-1}}{(x, y, s) \sim(y, x,-s)} \stackrel{S^{m-1}}{\rightarrow} \frac{\widetilde{T}}{(x, y) \sim(y, x)}, \quad \text { by } \quad \gamma[(x, y, s)]:=[(x, y)]
$$

The existence of an equivariant map $\widetilde{T} \rightarrow S^{m-1}$ is equivalent to the existence of a crosssection of $\gamma$; the existence of an equivariant homotopy between $\widetilde{f}_{0}$ and $\widetilde{f}_{1}$ is equivalent to the equivalence of the corresponding cross-sections of the bundle $\gamma$. Thus the existence of either can be checked using the methods of obstruction theory. In particular, the Whitney and the van Kampen obstructions $(\S 2, \S 4)$ are the first obstructions to the existence of a cross-section of the bundle $\gamma$ [Wu65]; the Whitney and the van Kampen-Wu invariants $(\S 2, \S 4)$ are the first obstructions to an equivariant homotopy of $\tilde{f}_{0}$ and $\widetilde{f}_{1}$ [Wu65].

The completeness of the Haefliger-Wu invariant. However trivial the 'deleted product necessary conditions' may seem, the above shows that they are very useful. Thus it is very interesting to find out for which cases they are also sufficient for embeddability and isotopy, i.e. for which cases the following assertions hold (the converses of which were just proved).

(e) If there exists an equivariant map $\Phi: \widetilde{N} \rightarrow S^{m-1}$, then $N$ embeds into $\mathbb{R}^{m}$.

(s) If there exists an equivariant map $\Phi: \widetilde{N} \rightarrow S^{m-1}$, then there exists an embedding $f: N \rightarrow \mathbb{R}^{m}$ such that $\tilde{f} \simeq_{e q} \Phi$.

(i) If $f_{0}, f_{1}: N \rightarrow \mathbb{R}^{m}$ are two embeddings and $\widetilde{f}_{0} \simeq_{e q} \widetilde{f}_{1}$, then $f_{0}$ and $f_{1}$ are isotopic.

Clearly, (s) and (i) are the surjectivity and the injectivity of $\alpha$. Obviously, (s) implies (e). From (e) it follows that if N TOP embeds into $\mathbb{R}^{m}$, then $N$ PL or DIFF embeds into $\mathbb{R}^{m}$ (in particular, that PL or DIFF embeddability of $N$ into $\mathbb{R}^{m}$ does not depend on PL or DIFF structures on $N$ ). Condition (i) has analogous corollary.

Thus the surjectivity and the injectivity of $\alpha$ are directly related to the Embedding and Knotting Problems.

The Haefliger-Weber Theorem 5.4. [Ha63, Theorem 1', We67, Theorems 1, 1'] For embeddings $N \rightarrow \mathbb{R}^{m}$ of either an $n$-polyhedron or a smooth $n$-manifold $N$ the HaefligerWu invariant is

$$
\text { bijective if } \quad 2 m \geq 3 n+4 \quad \text { and surjective if } 2 m=3 n+3 .
$$

The metastable dimension restrictions in the Haefliger-Weber Theorem 5.4 are sharp in the smooth case by the Trefoil Knot Example 3.4 (and other examples of smooth knots [Ha66]), and by Theorems 2.7.c, 2.7.d (because the PL embedability $N \rightarrow \mathbb{R}^{2 l+2}$ implies the existence of an equivariant map $\widetilde{N} \rightarrow S^{2 l+1} \subset S^{3 l-2}$ ). Such dimension restrictions appeared also in the PL cases of the classical theorems on embeddings of highly-connected manifolds and of Poincaré complexes (see Theorems 2.5, 2.6 and [Ru73, BM99, BM00]), but were later weakened to $m \geq n+3$. So since 1960's it was conjectured by Viro, 
Dranishnikov, Koschorke, Szucs, Schepin and others that also in Theorem 5.4 for the PL case and connected $N$ the metastable dimension restrictions can be weakened to $m \geq n+3$ (possibly at the price of adding the $p$-fold Haefliger-Wu invariant, see the subsection 'the generalized Haefliger-Wu invariant'). This turned out to be false not only for polyhedra (Examples 5.7.d and 5.9.c below) but even for PL manifolds (Examples 5.7.b, 5.7.c and 5.8.b below). So it is surprising that the metastable dimension restrictions can be weakened to $m \geq n+3$ for highly connected PL $n$-manifolds (less highly connected than in Theorems 2.6 and 2.8).

Theorem 5.5. [Sk02] For embeddings $N \rightarrow \mathbb{R}^{m}$ of is a closed d-connected PL $n$-manifold $N$ and $m \geq n+3$, the Haefliger-Wu invariant is

$$
\text { bijective if } 2 m \geq 3 n+3-d \quad \text { and surjective if } 2 m=3 n+2-d .
$$

For $d=1$ we need only homological 1-connectedness in Theorem 5.5.

Observe that Theorem 5.5 is not quite the result expected in the 1960's, and that its proof cannot be obtained by direct generalization of the Haefliger-Weber proof without invention of new ideas. This follows from the preceding discussion and the following two remarks.

(1) The PL case of Haefliger-Weber Theorem 5.4 holds for polyhedra, but Theorem 5.5 holds only for highly enough connected PL manifolds.

(2) The same $(3 n-2 m+2)$-connectedness assumption as in the surjectivity part of Theorem $5.5(2 m \geq 3 n+2-d \Leftrightarrow d \geq 3 n-2 m+2)$ appeared in the Hudson PL version of the Browder-Haefliger-Casson-Sullivan-Wall Embedding Theorem for closed manifolds (roughly speaking, it states that a homotopy equivalence between PL manifolds is homotopic to a PL embedding, and it was proved by engulfing) [Hu67]. This assumption was soon proved to be superfluous (by surgery) [Ha68, Hu70']. So it is natural to expect that the $(3 n-2 m+3)$-connectedness assumption in Theorem 5.5 is superfluous (Theorem 5.5 is proved using generalization of the engulfing approach). However, Non-injectivity Examples 5.7.b,c of the next subsection show that this assumption is sharp.

In this paper we sketch the proof of the surjectivity in the Haefliger-Weber Theorem 5.4 in the PL case and present the idea of the proof of Theorem 5.5.

Most of the results of $\S 2, \S 3$ and $\S 4$ are corollaries of Theorems 5.4 and 5.5, although some of them were originally proved independently (sometimes in a weaker form).

Corollary 5.6. If $N$ is a homologically 1-connected closed smooth n-manifold, then $\alpha_{D I F F}^{m}(N)$ is injective for $2 m=3 n+2, n=4 s+2$, and surjective for $2 m=3 n+1$, $n=4 s+3$.

Sketch of the proof. Theorem 5.5 and smoothing theory [Ha67, 1.6, Ha, 11.1] imply the following.

Let $N$ be a closed $d$-connected (for $d=1$, just homologically 1-connected) smooth $n$ manifold and $m \geq n+3$.

If $2 m \geq 3 n+2-d$, then for each $\Phi \in \pi_{e q}^{m-1}(\widetilde{N})$ there is a PL embedding $f: N \rightarrow \mathbb{R}^{m}$ smooth outside a point and such that $\alpha(f)=\Phi$; a complete obstruction to the smoothing of $f$ lies in $C_{n-1}^{m-n}$.

If $2 m \geq 3 n+3-d$, then any two smooth embeddings $f_{0}, f_{1}: N \rightarrow \mathbb{R}^{m}$ such that $\alpha\left(f_{0}\right)=\alpha\left(f_{1}\right)$ can be joined by a PL isotopy, which is smooth outside a point; a complete obstruction to the smoothing of such a PL isotopy lies in $C_{n}^{m-n}$. 
The Corollary follows from these assertions and $C_{4 k-2}^{2 k}=0$ [Ha66, 8.15, Mi72', Corollary C]. (There is a misprint in $[\mathrm{Ha} 66,8.15]$ : instead of ' $C_{4 k-2}^{3 k}=0$ ' it should be ' $C_{8 k-2}^{4 k}=$ $\left.0^{\prime}.\right)$

In Theorem 5.5 the surjectivity is not interesting for $m<\frac{5 n+6}{4}$. Indeed, $\frac{5 n+6}{4}>\frac{3 n+2-d}{2}$ implies that $d>\frac{n}{2}-1$ and $n \geq 6$; hence $N$ is a homotopy sphere, so $N \cong S^{n}$ and the surjectivity in Theorem 5.5 is trivial. But the proof is not simpler for $m \geq \frac{5 n+6}{4}$; the proof can also be considered as a step towards the analogue of Theorem 5.5 for embeddings into manifolds, which is interesting even for $m<\frac{5 n+6}{4}$. An analogous remark can be made about the injectivity in Theorem 5.5.

The Haefliger-Weber Theorem 5.4 has relative and approximative versions [Ha63, 1.7.2, We67, Theorems 3 and 7, RS98], which require that constructed embedding or isotopy extend given one or is close to given one. But Theorem 5.5 has such versions only under some additional assumptions.

An interesting corollary of [Ku30, Cl34, Cl37] was deduced in [Wu65] for graphs and in [Sk98] for the general case:

a Peano continuum $N$ embeds into $\mathbb{R}^{2}$ if and only if there exists an equivariant map $\widetilde{N} \rightarrow S^{1}$.

An interesting corollary of [MA41] was deduced in [Wu65]:

embeddings $f, g: N \rightarrow \mathbb{R}^{2}$ of a Peano continuum $N$ are isotopic if and only if $\widetilde{f} \simeq_{e q} \widetilde{g}$.

The incompleteness of the Haefliger-Wu invariant. Clearly, $\widetilde{S^{n}} \simeq_{e q} S^{n}$. Therefore the Haefliger-Wu invariant is not injective in codimension 2 (e.g. for knots in $\mathbb{R}^{3}$ ) and any smoothly non-trivial knot $S^{n} \rightarrow \mathbb{R}^{m}$ demonstrates the non-injectivity of $\alpha_{D I F F}^{m}\left(S^{n}\right)$. The deleted product necessary conditions for embeddability or for isotopy do not reflect either the ambience of isotopy or the distinction between the DIFF and PL (or TOP) categories. The same assertions hold for generalized Haefliger-Wu invariants (see below) or 'isovariant maps invariants' [Ha63, Ad93, §7].

Let us present another examples. All the examples in this subsection hold for each set of the parameters $k, l, m, n, p$ satisfying to the conditions in the statement.

Non-injectivity Examples 5.7. The following maps are not injective:

$$
\begin{gathered}
\text { (a) } \alpha^{3 l}\left(S^{2 l-1} \sqcup S^{2 l-1} \sqcup S^{2 l-1}\right), \quad \alpha^{3 l}\left(S^{2 l-1} \sqcup S^{2 l-1}\right) \quad \text { and } \quad \alpha_{D I F F}^{3 l}\left(S^{2 l-1}\right) ; \\
(b) \quad \alpha^{6 k}\left(S^{p} \times S^{4 k-1}\right) \quad \text { for } p<k \quad \text { [Sk02]; } \\
(c) \quad \alpha^{3 l+1}\left(S^{1} \times S^{2 l-1}\right), \quad \text { if } l+1 \text { is not a power of } 2[S k] ; \\
\text { (d) } \alpha_{P L}^{m}\left(\left(S^{n} \vee S^{n}\right) \sqcup S^{2 m-2 n-3}\right) \quad \text { for } n+2 \leq m \leq(3 n+3) / 2 \quad \text { [Sk02]. }
\end{gathered}
$$

The Non-surjectivity Example 5.7.a follows from the Borromean Rings, the Whitehead Link and the Trefoil Knot Examples of $§ 3$. The Non-injectivity Examples 5.7.b, 5.7.c and 5.7.d are constructed in $\S 6$, below and in $\S 7$, respectively.

The construction (but not the proof $[\mathrm{Sk}]$ ) of Example 5.7.c is very simple and explicit.

Construction of Example 5.7.c in the PL case. Add a strip to the Whitehead link $\omega_{0, P L}$, i.e. extend it to an embedding

$$
\omega_{0}^{\prime}: S^{0} \times S^{2 l-1} \bigcup_{S^{0} \times D_{+}^{2 l-1}=\partial D_{+}^{1} \times D_{+}^{2 l-1}} D_{+}^{1} \times D_{+}^{2 l-1} \rightarrow \mathbb{R}^{3 l} .
$$


This embedding contains connected sum of the components of the Whitehead link. The union of $\omega_{0}^{\prime}$ and the cone over the connected sum forms an embedding $D_{+}^{1} \times S^{2 l-1} \rightarrow \mathbb{R}_{+}^{3 l+1}$. This latter embedding can clearly be shifted to a proper embedding. The PL Whitehead torus $\omega_{1, P L}: S^{1} \times S^{2 l-1} \rightarrow \mathbb{R}^{3 l+1}$ is the union of this proper embedding and its mirror image with respect to $\mathbb{R}^{3 l} \subset \mathbb{R}^{3 l+1}$. Cf. definition of $\mu^{\prime}$ in $\S 6$.

It is easy to prove that $\alpha\left(\omega_{1, P L}\right)=\alpha\left(f_{0}\right)$, where $f_{0}$ is the standard embedding [Sk]. It is non-trivial that $\omega_{1, P L}$ is not PL isotopic to the standard embedding when $l+1$ is not a power of 2. The Non-injectivity Example 5.7.a for $\alpha^{3 l}\left(S^{2 l-1} \sqcup S^{2 l-1}\right)$ is based on the linking coefficient. The Non-injectivity Example 5.7.c is much more complicated because $S^{1} \times S^{2 l-1}$ is connected, so the linking coefficient cannot be defined (the linking coefficient for the restriction to $S^{0} \times S^{2 l-1}$ gives the weaker Non-injectivity Example 5.7.b); thus a new invariant $[\mathrm{Sk}]$ is required.

Non-surjectivity Examples 5.8. For $m \geq n+3$ the following maps are not surjective: (a) $\alpha^{m}\left(S^{n} \sqcup S^{n}\right)$, if $\Sigma^{\infty}: \pi_{n}\left(S^{m-n-1}\right) \rightarrow \pi_{2 n+1-m}^{S}$ is not epimorphic, e.g. for

$$
\begin{array}{ccccccc}
n & 6 & 9 & 12 & 13 & 14 & 21 \\
m & 10=\frac{3 n+2}{2} & 13=\frac{3 n-1}{2} & 18=\frac{3 n}{2} & 19=\frac{3 n-1}{2} & 22=\frac{3 n+2}{2} & 31=\frac{3 n-1}{2}
\end{array}
$$

(b) $\alpha^{m}\left(S^{1} \times S^{n-1}\right)$, if $m-n$ is odd and $\Sigma^{\infty}: \pi_{n-1}\left(S^{m-n}\right) \rightarrow \pi_{2 n-m-1}^{S}$ is not epimorphic [Sk02], e.g. for

$$
\begin{array}{ccccccc}
n & 7 & 10 & 13 & 14 & 15 & 22 \\
m & 10=\frac{3 n-1}{2} & 13=\frac{3 n-4}{2} & 18=\frac{3 n-3}{2} & 19=\frac{3 n-4}{2} & 22=\frac{3 n-1}{2} & 31=\frac{3 n-4}{2} \\
& & & &
\end{array}
$$

For $n \leq k+2$ the stable suspension mapping is denoted by $\Sigma^{\infty}: \pi_{n+k}\left(S^{n}\right) \rightarrow$ $\pi_{2 k+2}\left(S^{k+2}\right)=\pi_{k}^{S}$.

The Non-surjectivity Example 5.8.a follows from the formula $\alpha= \pm \Sigma^{\infty} \lambda_{12}$ and the construction of a link with the prescribed linking coefficient of $\S 3$. The Non-surjectivity Example 5.8.b is constructed in $\S 6$. The Non-surjectivity Example 5.8.c follows because $\alpha_{P L}^{6 k+1}\left(S^{2 k} \times S^{2 k}\right)$ is bijective by Theorem 5.5 (or by [Bo71, Ha62"]) but by the Haefliger Torus Example 3.5 there exists a PL embedding $S^{2 k} \times S^{2 k} \rightarrow \mathbb{R}^{6 k+1}$ that is not PL isotopic to a smooth embedding.

Links give many other examples of the non-injectivity and the non-surjectivity of $\alpha$. From a link example, by gluing an arc joining connected components we can obtain a highly connected polyhedral example.

Non-embeddability Examples 5.9. There exists an equivariant map $\widetilde{N} \rightarrow S^{m-1}$ but $N$ does not CAT embed into $\mathbb{R}^{m}$ (hence $\alpha_{C A T}^{m}(N)$ is not surjective) for

(a) $C A T=D I F F, m=n+3, n \in\{8,9,10,16\}$ and a certain homotopy $n$-sphere $N$;

(b) CAT=DIFF, $m=6 k-1, n=4 k$ and a certain (almost parallelizable $(2 k-1)$ connected) $n$-manifold $N$;

(c) $C A T=P L, \max \{3, n\} \leq m \leq \frac{3 n+2}{2}$ and a certain n-polyhedron $N$ [MS67, SS92, FKT94, SSS98, GS06]. 
The Non-Embeddability Example 5.9.a follows from the existence of a homotopy $n$ sphere $N$ which is non-embeddable in codimension 3 [HLS65, Le65, cf. Re90, §2, MT95, pp. 407-408] (because $N \cong S^{n}$ topologically and so $\widetilde{N} \simeq_{e q} \widetilde{S^{n}}$ ). The Non-Embeddability Example 5.9.b follows from Theorems 2.7.c and 2.7.d. The Non-Embeddability Example 5.9.c is proved in $\S 7$.

In [Sk98] it is proved that

although the 3-adic solenoid $\Sigma$ (i.e. the intersection of infinite sequence of filled-tori, each inscribed in the previous one with degree 3) does not embed into $\mathbb{R}^{2}$, nevertheless there exists an equivariant map $\widetilde{\Sigma} \rightarrow S^{1}$.

We conjecture that there exists a non-planar tree-like continuum $N$, for which there are no equivariant maps $\widetilde{N} \rightarrow S^{1}$.

The Generalized Haefliger-Wu invariant. The Borromean Rings Example 3.2 suggests that one can introduce an obstruction to embeddability, analogous to the deleted product obstruction (and the van Kampen obstruction, see $\S 4$ ) but deduced from a triple, quadruple, etc. product. Moreover, the vanishing of this obstruction should be sufficient for embeddability even when this is not so for the deleted product obstruction. Such an obstruction can indeed be constructed as follows, cf. [Kr00]. Suppose that $G$ is a subgroup of the group $S_{p}$ of permutations of $p$ elements and let

$\widetilde{N}_{G}=\left\{\left(x_{1}, \ldots, x_{p}\right) \in N^{p} \mid x_{i} \neq x_{\sigma(i)}\right.$ for each non-identity element $\left.\sigma \in G, i=1, \ldots, p\right\}$.

The space $\widetilde{N}_{G}$ is called the deleted $G$-product of $N$. The group $G$ obviously acts on the space $\widetilde{N}_{G}$. For an embedding $f: N \rightarrow \mathbb{R}^{m}$ the map $\widetilde{f}_{G}: \widetilde{N}_{G} \rightarrow \widetilde{\mathbb{R}}_{G}$ is well-defined by the formula $\widetilde{f}_{G}\left(x_{1}, \ldots, x_{p}\right)=\left(f x_{1}, \ldots, f x_{p}\right)$. Clearly, the map $\widetilde{f}_{G}$ is $G$-equivariant. Then we can define the $G$-Haefliger-Wu invarant

$$
\alpha_{G}=\alpha_{G}^{m}(N): \operatorname{Emb}^{m}(N) \rightarrow\left[\widetilde{N}_{G}, \widetilde{\mathbb{R}^{m}}\right]_{G} \quad \text { by } \quad \alpha_{G}(f)=\left[\widetilde{f}_{G}\right] .
$$

The deleted G-product obstruction for the embeddability of $N$ in $\mathbb{R}^{m}$ is the existence of a $G$-equivariant map $\Phi: \widetilde{N}_{G} \rightarrow{\widetilde{\mathbb{R}^{m}}}_{G}$.

This approach works well in link theory (the simplest example is the classification of 'higher-dimensional Borromean rings' [Ha62', $\S 3$, Ma90, Proposition 8.3] by means of $\alpha_{S_{3}}^{m}$ ). Surprisingly, in contrast to that, the Non-injectivity Examples 5.7 (except for the example of the Borromean rings of 5.7.a) demonstrate the non-injectivity of $\alpha_{G}$ for each $G$ : in their formulations $\alpha$ can be replaced by $\alpha_{G}$ for each $G$. This follows by the construction of these examples. Clearly, if $\alpha$ is not surjective, then neither is $\alpha_{G}$ for each $G$. Under the conditions of the Non-embeddability Examples 5.9 property (e) is false even if we replace the $\mathbb{Z}_{2}$-equivariant map $\widetilde{N} \rightarrow S^{m-1}$ by a $G$-equivariant map $\widetilde{N}_{G} \rightarrow{\widetilde{\mathbb{R}^{m}}}_{G}$.

Historical remarks. A particular case of Theorem 5.4 (Theorem 4.1) was discovered by Van Kampen [Ka32]. But Van Kampen's proof of the sufficiency in Theorem 4.1 contained a mistake. However, he modified his argument to prove the PL case of Theorem 2.2.a. Based on the idea of the Whitney trick invented in [Wh44], Shapiro and Wu completed the proof [Sh57, Wu58]. Subsequently their argument was generalized by Haefliger and Weber (using some ideas of Shapiro and Zeeman) in order to prove the Haefliger-Weber 
Theorem 5.4. The second part of the Weber proof was simplified in [Sk98] using the idea of the Freedman-Krushkal-Teichner Lemma 4.3.

The Whitney trick, on which the proof of sufficiency in Theorem 4.1 for $n \geq 3$ is based, cannot be performed for $n=2$ [KM61, La96]. Sarkaria has found a proof of the case $n=1$ of Theorem 4.1 based on 1-dimensional Whitney trick [Sa91'] (the author is grateful to K. Sarkaria and M. Skopenkov for indicating that the argument in [Sa91'] is incomplete). Sarkaria also asked whether the sufficiency in Theorem 4.1 holds for the case $n=2$. Freedman, Krushkal and Teichner have constructed an example showing that it does not [FKT94].

The dimension restriction $2 m \geq 3 n+3$ in the Haefliger-Weber Theorem 5.4 comes from the use of the Freudenthal Suspension Theorem, the Penrose-Whitehead-ZeemanIrwin Embedding Theorem 2.6.c, a relative version of the Zeeman Unknotting Theorem 2.5.a and general position arguments ( $\S 8$. Toruńczyk and Spież showed that one can try to relax the restriction coming from the Zeeman Unknotting Theorem (using relative regular neighborhoods) and those coming from the Freudenthal Suspension Theorem (using Whitehead's 'hard part' of the Freudenthal Suspension Theorem and the Whitehead higher-dimensional finger moves [FF89, §10]; note that application of higher-dimensional finger moves in this situation was first suggested by Schepin) [Sp90, ST91, see also DRS91, DRS93]. This was the reason why in 1992 Dranishnikov conjectured the surjectivity in the Haefliger-Weber Theorem 5.4 for $2 m=3 n+2$. However, Segal and Spież constructed a counterexample (using the same higher-dimensional finger moves) - a weaker version of Non-Embeddability Example 5.9.c [SS92]. Their construction used the Adams theorem on Hopf invariant one, and therefore has exceptions corresponding to the exceptional values $1,3,7$. In 1995 the author suggested how to remove the codimension 2 exceptions. Subsequently, this idea was generalized independently by Segal-Spież and the author to obtain a simplification of [SS92] which did not use the Adams theorem, and therefore has no exceptions [SSS98]. This simplified construction leads to Non-Embeddability Example 5.9.c.

\section{ON THE DELETED PRODUCT OF THE TORUS}

\section{Proof of the Deleted Product Lemma 5.3.a,b.}

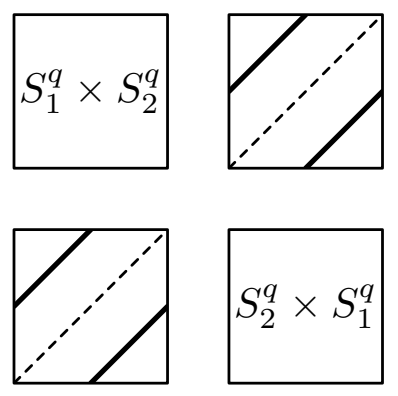

Figure 6.0

Proof of the Deleted Product Lemma 5.3.a. We have

$$
\widetilde{S^{p} \sqcup S^{q}} \simeq_{e q} S^{p} \times S^{q} \sqcup S^{p} \times S^{q} \sqcup S^{p} \sqcup S^{q},
$$


where the involution on the right-hand term exchanges antipodes in $S^{p}$ and in $S^{q}$, as well as the corresponding points from the two copies of $S^{p} \times S^{q}$ (Figure 6.0). Therefore analogously to the definition of the $\alpha$-invariant in $\S 3$ we have

$$
\pi_{e q}^{m-1}\left(\widehat{S^{p} \sqcup S^{q}}\right) \cong\left[S^{p} \times S^{q}, S^{m-1}\right] \cong \pi_{p+q+1-m}^{S} \quad \text { for } \quad m-2 \geq p, q
$$

Recall that the equivariant Stiefel manifold $V_{m n}^{e q}$ is the space of equivariant maps $S^{n-1} \rightarrow S^{m-1}$. Denote by $a_{k}: S^{k} \rightarrow S^{k}$ the antipodal map.

The Torus Lemma 6.1. For $p \leq q$ and $m \geq p+q+3$ there exist groups $\Pi_{p, q}^{m-1}$ and $\Pi_{q, p}^{m-1}$, a group structure on $\pi_{e q}^{m-1}\left(\widehat{S^{p} \times S^{q}}\right)$, and homomorphisms $\sigma, \gamma, \rho, \tau$ and $\alpha^{\prime}$ forming the diagram below, in which the right-hand square commutes and the left-hand square either commutes or anticommutes. The homomorphisms $\sigma, \gamma, \rho$ and $\mathrm{pr}_{1}$ are isomorphisms under the dimension restriction $m \geq A$, where $A$ is shown near the notation of a map.

$$
\begin{aligned}
& \pi_{q}\left(V_{m-q, p+1}\right) \underset{\tau \frac{3 q}{2}+p+2}{\longrightarrow} K T_{p, q}^{m} \quad \underset{\alpha}{\longrightarrow} \quad \pi_{e q}^{m-1}\left(\widetilde{S^{p} \times S^{q}}\right) \\
& \downarrow \rho \frac{3 q}{2}+p+2 \quad \downarrow \alpha^{\prime} \quad \downarrow \gamma p+q+3 \\
& \pi_{q}\left(V_{m-q, p+1}^{e q}\right) \underset{\sigma \frac{3 q+p}{2}+2}{\longrightarrow} \Pi_{p q}^{m-1} \stackrel{\mathrm{pr}_{1} q+2 p+2}{\longleftarrow} \Pi_{p, q}^{m-1} \oplus \Pi_{q, p}^{m-1}
\end{aligned}
$$

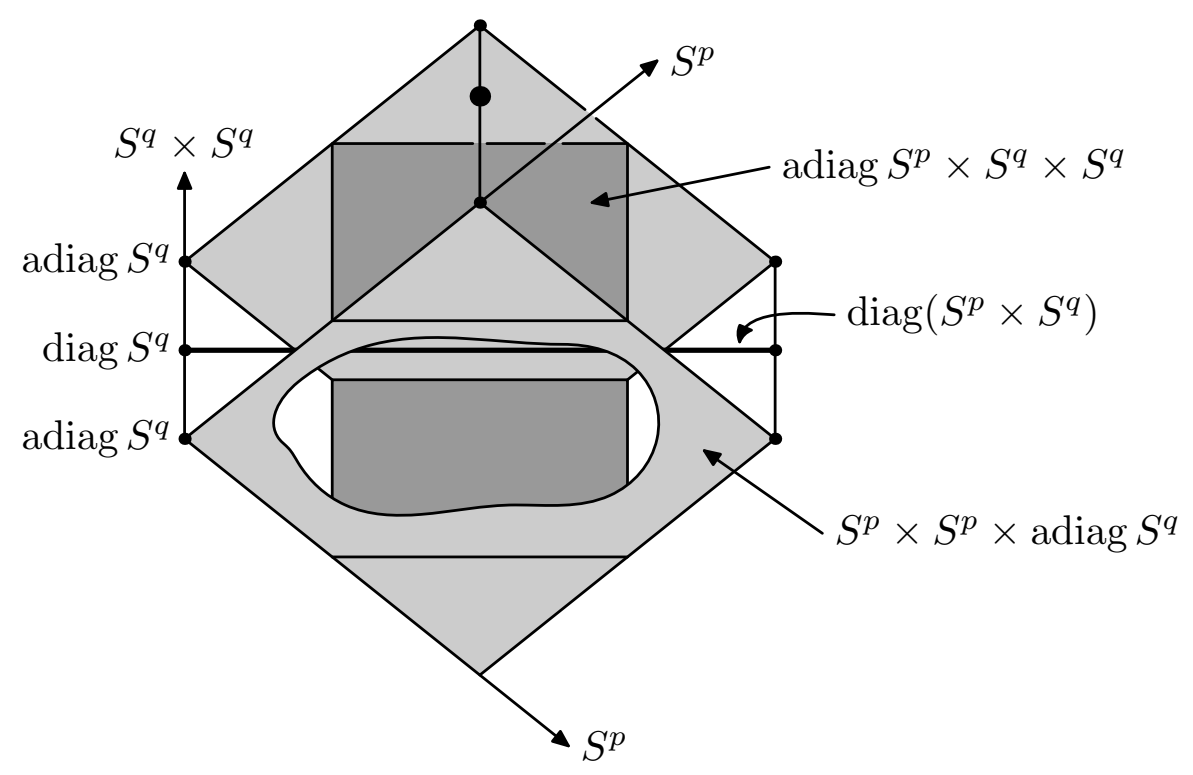

Figure 6.1

Proof. There is an equivariant deformation retraction

$$
H_{1}: \widetilde{S^{p} \times S^{q}} \rightarrow \operatorname{adiag} S^{p} \times S^{q} \times S^{q} \bigcup_{\operatorname{adiag} S^{p} \times \operatorname{adiag} S^{q}} S^{p} \times S^{p} \times \operatorname{adiag} S^{q},
$$


where adiag is antidagonal (Figure 6.1). More precisely, for non-antipodal points $x$ and $y$ of $S^{k}$ and $t \in[0,1]$, let $[x, y, t]=\frac{(1-t) x+t y}{|(1-t) x+t y|}$. Define the deformation $H_{t}: \widetilde{S^{p} \times S^{q}} \rightarrow \widetilde{S^{p} \times S^{q}}$ by

$H\left(x, y, x_{1}, y_{1}\right)=\left\{\begin{array}{ll}\left([x, y, t],[y, x, t],\left[x_{1}, y_{1}, 2 \delta t\right],\left[y_{1}, x_{1}, 2 \delta t\right]\right) & |x, y| \geq\left|x_{1}, y_{1}\right| \\ \left([x, y, 2(1-\delta) t],[y, x, 2(1-\delta) t],\left[x_{1}, y_{1}, t\right],\left[y_{1}, x_{1}, t\right]\right) & \left|x_{1}, y_{1}\right| \geq|x, y|\end{array}\right.$,

where $\delta=\frac{|x, y|}{\left|x_{1}, y_{1}\right|+|x, y|}$.

Define $\Pi_{p q}^{m-1}:=\pi_{e q}^{m-1}\left(S^{p} \times S^{2 q}\right)$, where the involution on $S^{p} \times S^{2 q}$ is $a_{p} \times t_{q}$ and $t_{q}: S^{2 q} \rightarrow S^{2 q}$ is the symmetry with respect to $S^{q} \subset S^{2 q}$. The group structure on $\Pi_{p q}^{m-1}$ is defined as follows. For equivariant maps $\varphi, \psi: S^{p} \times S^{2 q} \rightarrow S^{m-1}$ define the map $\varphi+\psi: S^{p} \times S^{2 q} \rightarrow S^{m-1}$ on $x \times S^{2 q}$ to be the ordinary sum of the restrictions of $\varphi$ and $\psi$ to $x \times S^{2 q}$. Analogously, define the unity and the inverse of $\varphi$ on $x \times S^{2 q}$ to be the ordinary unity and the ordinary inverse of $\left.\varphi\right|_{x \times S^{2 q}}$.

Let $v_{q}: S^{q} \times S^{q} \rightarrow \frac{S^{q} \times S^{q}}{S^{q} \vee S^{q}} \cong S^{2 q}$ be the quotient map, cf. $\S 3$. Consider the involution $(s, x, y) \rightarrow(-s, y, x)$ on $S^{p} \times S^{q} \times S^{q}$. For $m \geq p+q+3$ by general position we have a $1-1$ correspondence

$$
\left(\operatorname{id}_{S^{p}} \times v_{q}\right)^{*}: \pi_{e q}^{m-1}\left(S^{p} \times S^{q} \times S^{q}\right) \cong \Pi_{p q}^{m-1} .
$$

One can check that the involution on $S^{q} \times S^{q}$ exchanging factors corresponds to $t_{q}$.

Consider the restrictions of an equivariant map

$$
\widetilde{S^{p} \times S^{q}} \rightarrow S^{m-1} \quad \text { to } \operatorname{adiag} S^{p} \times S^{q} \times S^{q} \quad \text { and to } S^{p} \times S^{p} \times \operatorname{adiag} S^{q} .
$$

Define the map $\gamma$ to be the direct sum of the compositions of such restrictions and the isomorphisms $\left(\operatorname{id}_{S^{p}} \times v_{q}\right)^{*}$ and $\left(\operatorname{id}_{S^{q}} \times v_{p}\right)^{*}$. If

$$
\operatorname{dim}\left(\operatorname{adiag} S^{p} \times \operatorname{adiag} S^{q}\right)=p+q \leq(m-1)-2,
$$

then $\gamma$ is a $1-1$ correspondence by general position and the Borsuk Homotopy Extension Theorem. Take the group structure on $\pi_{e q}^{m-1}\left(\widetilde{S^{p} \times S^{q}}\right)$ induced by $\gamma$. Then $\gamma$ is an isomorphism.

By general position, for $2 p+q \leq m-2$ we have $\Pi_{q p}^{m-1}=0$, hence $\mathrm{pr}_{1}$ is an isomorphism.

Let $\alpha^{\prime}$ be the map corresponding under the isomorphism $\left(\operatorname{id}_{S^{p}} \times v_{q}\right)^{*}$ to the map

$$
S^{p} \times S^{q} \times S^{q} \rightarrow S^{m-1} \quad \text { defined by } \quad(s, x, y) \mapsto \widetilde{f}((s, x),(-s, y)) .
$$

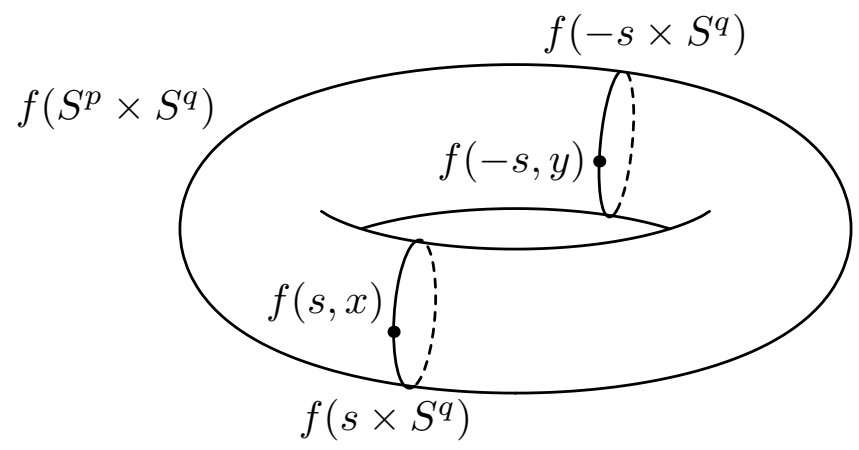


Figure 6.2

Clearly, the right-hand square of the diagram commutes.

The map $\tau$ was defined after Theorem 3.9.

Recall that $\rho$ is the inclusion-induced homomorphism. By [HH62, Sk], $\rho$ is an isomorphism for $m \geq \frac{3 q}{2}+p+2$.

An element $\varphi \in \pi_{q}\left(V_{m-q, p+1}^{e q}\right)$ can be considered as a map $\varphi: S^{p} \times S^{q} \rightarrow S^{m-q-1}$ such that $\varphi(-x, y)=-\varphi(x, y)$ for each $x \in S^{p}$. Let $\sigma(\varphi)$ be the $q$-fold $S^{p}$-fiberwise suspension of such a map $\varphi$, i.e. $\left.\sigma(\varphi)\right|_{x \times S^{2 q}}=\Sigma^{q}\left(\left.\varphi\right|_{x \times S^{q}}\right)$. It is easy to see that $\sigma$ is a homomorphism.

The (anti)commutativity of the left-hand square is proved for $p=0$ using the representation $S^{m-1} \cong S^{m-q-1} * S^{q-1}$ and deforming $\alpha^{\prime} \tau(\varphi)$ to the $S^{p}$-fiberwise suspension $\sigma(\varphi)$ of $\varphi\left[\right.$ Ke59]. For $p>0$ we apply this deformation for each $x \in S^{p}$ independently.

It remains to prove that $\sigma$ is an isomorphism for $m \geq \frac{3 q+p}{2}+2 \geq p+q+2$. For this observe that $\sigma$ is a composition

$$
\pi_{q}\left(V_{m-q, p+1}^{e q}\right)=\pi_{e q}^{m-q-1}\left(S^{p} \times S^{q}\right) \stackrel{\Sigma^{q}}{\rightarrow} \pi_{e q}^{m-1}\left(\Sigma^{q}\left(S^{p} \times S^{q}\right)\right) \stackrel{\mathrm{pr}^{*}}{\rightarrow} \Pi_{p q}^{m-1}
$$

Here the involution on $S^{p} \times S^{q}$ is $a_{p} \times \operatorname{id}_{S^{q}}$, the involutions on $\Sigma^{q} S^{q}$ and on $\Sigma^{q}\left(S^{p} \times S^{q}\right)$ are the 'suspension' involutions over $\operatorname{id}_{S^{q}}$ and $a_{p} \times \operatorname{id}_{S^{q}}$; the map

$$
\operatorname{pr}: S^{p} \times \Sigma^{q} S^{q}=S^{p} \times \frac{S^{q} \times D^{q}}{S^{q} \times y, y \in \partial D^{q}} \rightarrow \frac{S^{p} \times S^{q} \times D^{q}}{S^{p} \times S^{q} \times y, y \in \partial D^{q}}=\Sigma^{q}\left(S^{p} \times S^{q}\right)
$$

is a quotient map (Figure 6.3). The $S^{p}$-fiberwise group structures on $\pi_{e q}^{m-q-1}\left(S^{p} \times S^{q}\right)$ and on $\pi_{e q}^{m-1}\left(\Sigma^{q}\left(S^{p} \times S^{q}\right)\right)$ are defined analogously to that on $\Pi_{p, q}^{m-1}$. By the equivariant version of the Freudenthal Suspension Theorem, it follows that the above $\Sigma^{q}$ is an isomorphism for $p+q \leq 2(m-q-1)-2$. The non-trivial preimages of pr are $S^{p} \times\left[S^{q} \times y\right], y \in \partial D^{q}$. Their union is homeomorphic to $S^{p} \times \partial D^{q}$. Since $\operatorname{dim}\left(S^{p} \times \partial D^{q}\right)=p+q-1$, by general position it follows that $\mathrm{pr}^{*}$ is an isomorphism for $p+q-1 \leq m-3$. Therefore $\sigma$ is an isomorphism for $m \geq \frac{3 q+p}{2}+2 \geq p+q+2$.

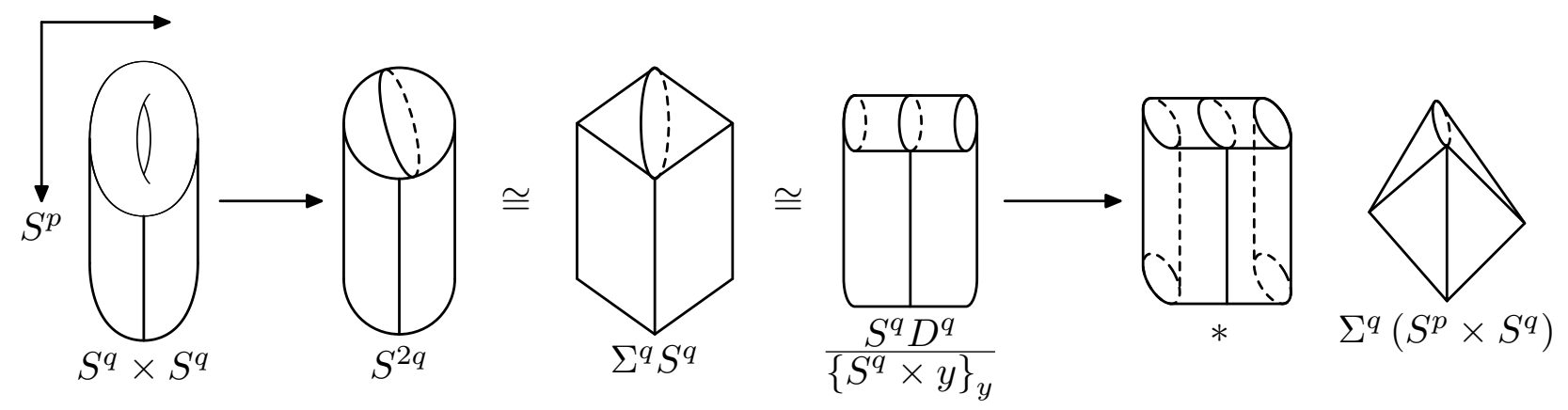

Figure 6.3

Note that the above map $\left(\operatorname{id}_{S^{p}} \times v_{q}\right)^{*}$ is an isomorphism also for $m=p+q+2$. This is proved using the cofibration exact sequence of the pair $\left(S^{p} \times S^{q} \times S^{q}, S^{p} \times\left(S^{q} \vee S^{q}\right)\right)$ and a retraction

$$
\Sigma\left(S^{p} \times S^{q} \times S^{q}\right) \rightarrow \Sigma\left(S^{p} \times\left(S^{q} \vee S^{q}\right)\right)
$$

obtained from the retraction

$$
\text { id }_{S^{p}} \times r_{q}: S^{p} \times \Sigma\left(S^{q} \times S^{q}\right) \rightarrow S^{p} \times \Sigma\left(S^{q} \vee S^{q}\right)
$$

by shrinking the product of $S^{p}$ with the vertex of the suspension to a point. 
The Generalized Torus Lemma 6.2. [Sk02] (a) If

$$
s \geq 3, \quad p_{1} \leq \cdots \leq p_{s}, \quad n=p_{1}+\cdots+p_{s} \quad \text { and } \quad N=S^{p_{1}} \times \cdots \times S^{p_{s}},
$$

then the same assertion as in the Torus Lemma 6.1 holds for the following diagram:

$$
\begin{array}{rcccc}
\pi_{n-p_{1}}\left(V_{m-n+p_{1}, p_{1}+1}\right) & \underset{\tau}{l} & \operatorname{Emb}^{m}(N) & \vec{\alpha} & \pi_{e q}^{m-1}(\widetilde{N}) \\
\downarrow \rho \frac{3 n-p_{1}}{2}+2 & & \downarrow \alpha^{\prime} & & \downarrow \gamma 22 n-p_{1}-p_{2}+3 \\
\pi_{n-p_{1}}\left(V_{m-n+p_{1}, p_{1}+1}^{e q}\right) & \underset{\sigma \frac{3 n}{2}-p_{1}+2}{\longrightarrow} & \Pi_{p_{1}, n-p_{1}}^{m-1} & \stackrel{\operatorname{pr}_{1} 2 n-p_{2}+2}{\longleftarrow} & \oplus_{i} \Pi_{p_{i}, n-p_{i}}^{m-1}
\end{array}
$$

(b) Suppose that

$$
s \geq 3, \quad n=p_{1}+\cdots+p_{s}, \quad p_{1} \leq \cdots \leq p_{s} \quad \text { and } \quad m \geq 2 n-p_{1}-p_{2}+3
$$

(for $s=3$ and $C A T=D I F F$ assume also that $m \geq \frac{3 n}{2}+2$ ). Then

$$
\operatorname{Emb}^{m}\left(S^{p_{1}} \times \cdots \times S^{p_{s}}\right)=\oplus_{i=1}^{s} \pi_{n-p_{i}}\left(V_{m-n+p_{i}, p_{i}+1}\right) .
$$

Proof. Part (b) follows from part (a) and Theorems 5.4 and 5.5. The proof of (a) is analogous to the proof of the Torus Lemma 6.1. We shall only define $\tau$ and $\sigma$ and omit the details.

The map $\tau$ is defined as follows. An element $\varphi \in \pi_{n-p_{1}}\left(V_{m-n+p_{1}, p_{1}+1}\right)$ is represented by a map $S^{n-p_{1}} \times S^{p_{1}} \rightarrow S^{m-n+p_{1}-1}$. Consider the projections

$$
\operatorname{pr}_{1}: N \rightarrow S^{p_{1}} \times S^{p_{2}+\cdots+p_{s}}=S^{p_{1}} \times S^{n-p_{1}} \quad \text { and } \quad \operatorname{pr}_{2}: N \rightarrow S^{p_{2}} \times \cdots \times S^{p_{s}} .
$$

Analogously to the case $s=2$, define an embedding $\tau(\varphi)$ as the composition

$$
S^{p_{1}} \times S^{p_{2}} \times \cdots \times S^{p_{s}} \stackrel{\left(\varphi \circ \mathrm{pr}_{1}\right)}{\longrightarrow} \times \mathrm{pr}_{2} \partial D^{m-n+p_{1}} \times S^{p_{2}} \times \cdots \times S^{p_{s}} \subset \mathbb{R}^{m} .
$$

The map $\sigma$ is defined analogously to the case $s=2$ as the $S^{p_{1}}$-fiberwise $\left(n-p_{1}\right)$-fold suspension.

Denote $q_{1}:=n-p_{1}$. Equivalently, $\sigma$ is a composition

$$
\pi_{q_{1}}\left(V_{m-q_{1}, p_{1}+1}^{e q}\right)=\pi_{e q}^{m-q_{1}-1}\left(S^{p_{1}} \times S^{q_{1}}\right) \stackrel{\Sigma^{q_{1}}}{\rightarrow} \pi_{e q}^{m-1}\left(\Sigma^{q_{1}}\left(S^{p_{1}} \times S^{q_{1}}\right)\right) \stackrel{\mathrm{pr}^{*}}{\rightarrow} \Pi_{p_{1}, q_{1}}^{m-1} .
$$

Here the maps $\Sigma^{q_{1}}$ and $\mathrm{pr}^{*}$ are isomorphisms for $2 m \geq 3 n-2 p_{1}+4$ and $m \geq n+2$, respectively. Therefore $\sigma$ is an isomorphism for $m \geq 3 n / 2-p_{1}+2$.

Note that under the assumptions of the Generalized Torus Lemma 6.2.b we have $m \geq$ $\frac{3 n}{2}+2$ for $s \geq 4$. 


\section{Proof of the Non-surjectivity Example 5.7.b and the Non-injectivity Example 5.8.b.}

The Decomposition Lemma 6.3. [Sk02] For $m \geq 2 p+q+1 \geq q+3$ there is the following (anti)commutative diagram, in which the first and the third lines are exact. The map $\nu$ is epimorphic for $m-q$ even and $\operatorname{im} \nu$ is the subgroup of elements of order 2 for $m-q$ odd

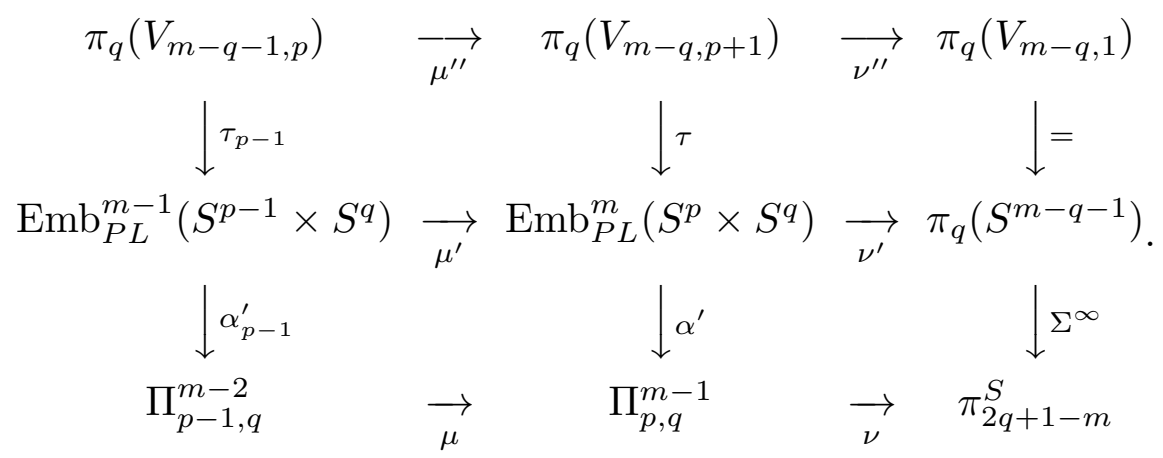

Of this Lemma only the right squares and the exactness at $\Pi_{p q}^{m-1}$ are used for the examples. The left squares are interesting in themselves and are useful elsewhere [Sk]. The definition of $\mu^{\prime}$ here is simpler than that in [Sk02].

Definition of the maps from the diagram. Let $\nu^{\prime \prime}$ and $\mu^{\prime \prime}$ be the homomorphisms induced by the 'forgetting the first $p$ vectors' bundle $V_{m-q, p+1} \stackrel{V_{m-q}-1, p}{\rightarrow} V_{m-q, 1}$.

For an embedding $f: S^{p} \times S^{q} \rightarrow \mathbb{R}^{m}$ let $\nu^{\prime}(f)$ be the linking coefficient of $\left.f\right|_{x \times S^{q}}$ and $\left.f\right|_{-x \times S^{q}}$ in $\mathbb{R}^{m}$.

Define the map $\nu: \Pi_{p, q}^{m-1} \rightarrow \Pi_{0, q}^{m-1} \cong \pi_{2 q-m+1}^{S}$ as 'the restriction over $* \times S^{2 q}$ '.

In order to define $\mu^{\prime}$ denote by $S^{p}=D_{+}^{p} \bigcup_{\partial D_{+}^{p}=S^{p-1}=\partial D_{-}^{p}} D_{-}^{p}$ the standard decomposition of $S^{p}$. Define analogously $\mathbb{R}_{ \pm}^{m}$ and $\mathbb{R}^{m-1}$. Add a strip to an embedding $f: S^{p-1} \times S^{q} \rightarrow$ $\mathbb{R}^{m-1}$, i.e. extend it to an embedding

$$
f^{\prime}: S^{p-1} \times S^{q} \bigcup_{S^{p-1} \times D_{+}^{q}=\partial D_{+}^{p} \times D_{+}^{q}} D_{+}^{p} \times D_{+}^{q} \rightarrow \mathbb{R}^{m} .
$$

Since $m \geq 2 p+q+1$, it follows that this extension is unique up to isotopy. The union of $f^{\prime}$ and the cone over the restriction of $f^{\prime}$ to the boundary forms an embedding $D_{+}^{p} \times$ $S^{q} \rightarrow \mathbb{R}_{+}^{m}$. This latter embedding can clearly be shifted to a proper embedding. Define $\mu^{\prime} f: S^{p} \times S^{q} \rightarrow \mathbb{R}^{m}$ to be the union of this proper embedding and its mirror image with respect to $\mathbb{R}^{m-1} \subset \mathbb{R}^{m}$.

Let us define the map $\mu$ first for the case $p=1$. For a map $\varphi: S^{2 q} \rightarrow S^{m-2}$ define the map $\mu \varphi$ to be the equivariant extension of the composition $D^{1} \times S^{2 q} \stackrel{\text { pr }}{\rightarrow} \Sigma S^{2 q} \stackrel{\Sigma \varphi}{\rightarrow} S^{m-1}$. In order to define the map $\mu$ for arbitrary $p$, replace

$$
\Pi_{p-1, q}^{m-2} \quad \text { and } \quad \Pi_{p q}^{m-1} \quad \text { by } \pi_{e q}^{m-2}\left(\Sigma^{q}\left(S^{p-1} \times S^{q}\right)\right) \text { and } \pi_{e q}^{m-1}\left(\Sigma^{q}\left(S^{p} \times S^{q}\right)\right),
$$

respectively (see the the proof of Torus Lemma 6.1). For an equivariant map $\varphi: \Sigma^{q}\left(S^{p-1} \times\right.$ $\left.S^{q}\right) \rightarrow S^{m-2}$ let $\mu \varphi$ be the composition

$$
\Sigma^{q}\left(S^{p} \times S^{q}\right)=\Sigma^{q}\left(\Sigma S^{p-1} \times S^{q}\right) \stackrel{\Sigma^{q}}{\rightarrow} \mathrm{pr} \Sigma^{q+1}\left(S^{p-1} \times S^{q}\right) \stackrel{\Sigma \varphi}{\rightarrow} \Sigma S^{m-2},
$$


where pr is the map from the proof of the Torus Lemma 6.1. Clearly, the definition for arbitrary $p$ agrees with that for $p=1$.

Note that all the maps of the Decomposition Lemma 6.3 except $\mu^{\prime}$ are defined for $m \geq p+q+3$.

The embedding $\mu^{\prime} f$ can also be defined by the Penrose-Whitehead-Zeeman-Irwin Embedding Theorem 2.6.c and its isotopy analogue. For $m \geq 2 p+q+2$ any embedding $f: S^{p-1} \times S^{q} \rightarrow S^{m-1}$ can be extended to a PL embedding $f_{ \pm}: D_{ \pm}^{p} \times S^{q} \rightarrow \mathbb{R}_{ \pm}^{m}$, uniquely up to isotopy. Then two embeddings $f_{+}$and $f_{-}$define an embedding $\mu^{\prime}(f): S^{p} \times S^{q} \rightarrow \mathbb{R}^{m}$.

Proof of the Decomposition Lemma 6.3. It is easy to check that both $\nu$ and $\mu$ are homomorphisms.

Clearly, the left-upper square of the diagram commutes.

Clearly, the right-upper square of the diagram commutes, see details in [Ti69, Lemma $3]$.

The right-bottom square of the diagram (anti)commutes by [Ke59, Lemma 5.1].

We prove the commutativity of the left-bottom square for $p=1$; the proof is analogous for the general case. Take an embedding $f: S^{0} \times S^{q} \rightarrow \mathbb{R}^{m-1}$. Then $\alpha \mu^{\prime} f=\mu \alpha_{0}^{\prime} f$ on $S^{0} \times S^{2 q}$. Also, for each $y \in S^{1} \times S^{q} \times S^{q}$ the points $\left(\alpha^{\prime} \mu^{\prime} f\right) y$ and $\left(\mu \alpha_{0}^{\prime} f\right) y$ are either both in the upper or both in the lower hemisphere of $S^{m-1}$. Hence $\alpha^{\prime} \mu^{\prime} f \simeq_{e q} \mu \alpha_{0}^{\prime} f$.

Let us prove the exactness at $\Pi_{p q}^{m-1}$. Clearly, $\nu \mu=0$. On the other hand, if $\Phi$ : $S^{p} \times S^{2 q} \rightarrow S^{m-1}$ is an equivariant map such that $\left.\Phi\right|_{* \times S^{2 q}}$ is null-homotopic, then by the Borsuk Homotopy Extension Theorem, $\Phi$ is equivariantly homotopic to a map which maps $* \times S^{2 q}$ and $a_{p}(*) \times S^{2 q}$ to antipodal points of $S^{m-1}$. By the Equivariant Suspension Theorem, the latter map is in $\operatorname{im} \mu$, since $p-1+2 q \leq 2(m-2)-1$. So $\operatorname{ker} \nu=\operatorname{im} \mu$.

Clearly, im $\nu$ consists of homotopy classes $\varphi \in \Pi_{0, q}^{m-1}$ extendable to a map $D^{1} \times S^{2 q} \rightarrow$ $S^{m-1}$. Such maps $\varphi$, considered as maps $\varphi: S^{2 q} \rightarrow S^{m-1}$ are exactly those which satisfy $a_{m-1} \circ \varphi \circ t_{q} \simeq \varphi$. The latter condition is equivalent to $(-1)^{m} \varphi=(-1)^{q} \varphi$ (for $m$ odd this follows by [Po85, complement to lecture 6, (10), p.264], since $h_{0}: \pi_{2 q}\left(S^{m-1}\right) \rightarrow$ $\pi_{2 q}\left(S^{2 m-3}\right)$ and $\left.2 q<2 m-3\right)$. So $\operatorname{im} \nu=\operatorname{ker}\left(1-(-1)^{m-q}\right)$.

Proof of the Non-surjectivity Example 5.8.b. Set $q=n-1 \leq m-4$. Look at the rightbottom square of the diagram from the Decomposition Lemma 6.3 and use the surjectivity of $\nu$ for $m-q$ even. The specific examples can be found using [To64, §14] (set $l=m-n=$ $m-q-1$ and $k=2 q+1-m)$.

Proof of the Non-injectivity Example 5.7.b. Since $p<k$, we have $m \geq 2 p+q+2$. Look at the right squares of the diagram from the Decomposition Lemma 6.3 and use Lemma 6.4 below.

Lemma 6.4. (a) $\Pi_{p q}^{m-1}$ is finite when $p+q+2 \leq m \leq 2 q$.

(b) The image of the restriction-induced homomorphism $\nu_{p}^{\prime \prime}: \pi_{4 k-1}\left(V_{2 k+1, p+1}\right) \rightarrow$ $\pi_{4 k-1}\left(S^{2 k}\right)$ is infinite for $p<2 k$.

Proof. Let us prove (a) by induction on $p$. The base of the induction is $p=0$, when $\Pi_{0 q}^{m-1} \cong \pi_{2 q}\left(S^{m-1}\right)$ is indeed finite. The inductive step of (a) follows by the induction hypothesis and the exactness of the bottom line from Decomposition Lemma 6.3.

In order to prove (b) for $p=0$ observe that the map $\nu_{0}$ is an isomorphism and $\pi_{4 k-1}\left(S^{2 k}\right)$ is infinite. Suppose that $p \geq 1$ and there is an infinite set $\left\{x_{i}\right\} \in \pi_{4 k-1}\left(V_{2 k+1, p}\right)$ 
with distinct $\nu_{p-1}^{\prime \prime}$-images. Consider the Serre fibration $S^{2 k+1-p} \rightarrow V_{2 k+1, p+1} \stackrel{\psi}{\rightarrow} V_{2 k+1, p}$ and the following segment of its exact sequence:

$$
\pi_{4 k-1}\left(V_{2 k+1, p+1}\right) \stackrel{\psi_{*}}{\rightarrow} \pi_{4 k-1}\left(V_{2 k+1, p}\right) \rightarrow \pi_{4 k-2}\left(S^{2 k-p}\right) .
$$

Since $\pi_{4 k-2}\left(S^{2 k-p}\right)$ is finite, by exactness it follows that the number of congruence classes of $\pi_{4 k-1}\left(V_{2 k+1, p}\right)$ modulo im $\psi_{*}$ is finite. Therefore an infinite number of the $x_{i}$ (we may assume all the $x_{i}$ ) lie in the same congruence class. By passing from $\left\{x_{i}\right\}$ to $\left\{x_{i}-x_{1}\right\}$ we may assume that this congruence class is the subgroup $\operatorname{im} \psi_{*}$ itself. Hence the inductive step follows from $\nu_{p}^{\prime \prime}=\nu_{p-1}^{\prime \prime} \psi_{*}$.

For a group $G$ let $G_{[k]}=G$ for $k$ even and let $G_{[k]}$ be the subgroup of $G$ formed by elements of order 2 for $k$ odd. For $m-q$ even, from the existence of a section $s$ : $\pi_{q}\left(V_{m-q, 1}\right) \rightarrow \pi_{q}\left(V_{m-q, 2}\right)$ it follows that $\nu^{\prime \prime}$ is epimorphic and hence $\nu^{\prime}$ is epimorphic. We also have $\operatorname{im} \nu=\operatorname{im} \nu^{\prime}=\operatorname{im} \nu^{\prime \prime}=\pi_{2 q-m+1,[m-q]}$ for $2 m \geq 3 q+4$. Note that $\operatorname{im} \nu=$ $\pi_{2 q-m+1,[m-q]}$ even for $2 m \leq 3 q+3$ but by Lemma 6.4.b $\operatorname{im} \nu^{\prime \prime} \neq \pi_{q}\left(S^{m-q-1}\right)_{[m-q]}$ for $2 m \leq 3 q+3$.

We conjecture that if $m-q$ is even $\geq 4$ and $2 m \geq 3 q+4$, then $\Pi_{1, q}^{m-1} \cong \pi_{2 q-m+2}^{S} \oplus$ $\pi_{2 q-m+1}^{S}$. We conjecture that in general $\Pi_{1 q}^{m-1}$ is adjoint to $\pi_{2 q-m+2,[m-q]}^{S} \oplus \pi_{2 q-m+1,[m-q]}^{S}$, unless $m=2 q+1$ and $q=2 l$ is even, when $\Pi_{1,2 l}^{4 l} \cong \mathbb{Z}_{2}$ (cf. the formula for $\pi_{q}\left(V_{m-q, 2}\right)$ before Theorem 3.10). Since $\operatorname{im} \nu=\pi_{2 q-m+1,[m-q]}^{S}$, by the Decomposition Lemma 6.3 the conjecture would follow from $\operatorname{coim} \mu \cong \pi_{2 q-m+2,[m-q]}^{S}$ (recall that we identify $\Pi_{0 q}^{m-2}=$ $\left.\pi_{2 q-m+2}^{S}\right)$.

\section{The Borromean RINGs AND the HaEfliger-Wu invariant}

All examples illustrating that the metastable dimension restrictions in embedding theorems are sharp have their origin in the Borromean Rings Example 3.2. So let us illustrate the idea of the Non-injectivity Example 5.7.d and the Non-embeddability Example 5.9.c by an alternative (comparatively to Example 3.2) construction of three circles embedded into $\mathbb{R}^{3}$ so that every pair of them is unlinked but all three are linked together. Our construction is based on the fact that the fundamental group is not always commutative.

Take two unknotted circles $\Sigma$ and $\bar{\Sigma}$ in $\mathbb{R}^{3}$ far away from each other. Embed in $\mathbb{R}^{3}-$ $(\Sigma \sqcup \bar{\Sigma})$ the Figure Eight, i.e. the wedge $C$ of two oriented circles so that the inclusion $C \subset \mathbb{R}^{3}-(\Sigma \sqcup \bar{\Sigma})$ induces an isomorphism of the fundamental groups. Take generators $a$ and $\bar{a}$ of $\pi_{1}(C) \cong \pi_{1}\left(\mathbb{R}^{3}-(\Sigma \sqcup \bar{\Sigma})\right)$ represented by the two arbitrarily oriented circles of the Figure Eight. Consider a map $S^{1} \rightarrow C \subset \mathbb{R}^{3}$ representing the element $a \bar{a} a^{-1} \bar{a}^{-1}$. By general position, there is an embedding $f: S^{1} \rightarrow \mathbb{R}^{3}$, very close to this map. It is easy to choose $f$ so that $\Sigma$ and $f\left(S^{1}\right), \bar{\Sigma}$ and $f\left(S^{1}\right)$ are unlinked (because $f$ induces the zero homomorphism of the 1-dimensional homology groups). Then $\Sigma, \bar{\Sigma}$ and $f\left(S^{1}\right)$ are as required (cf. Figure 7.1). Indeed, $\Sigma$ and $\bar{\Sigma}$ are unlinked by their definition. But $f$ induces a nonzero homomorphism of the fundamental groups. Therefore the three circles $\Sigma, \bar{\Sigma}$ and $f\left(S^{1}\right)$ are linked together.

Higher-dimensional Boromean rings can be constructed analogously using Whitehead products instead of commutators. 
Sketch of a counterexample to the relative versions of Theorem 4.1 for $n=2$ and to the surjectivity in the Haefliger-Weber Theorem 5.4 for $m=2 n=4$. Let

$$
N=D^{2} \sqcup D^{2} \sqcup D^{2} \quad \text { and } \quad A=\partial D^{2} \sqcup \partial D^{2} \sqcup \partial D^{2} .
$$

Let $A \subset S^{3} \cong \partial D^{4}$ be (generalized) Borromean rings.

Since all the three rings are linked, it follows that the embedding $A \rightarrow \partial D^{4}$ cannot be extended to an embedding $N \rightarrow D^{4}$. But since each pair of Borromean rings is unlinked, it follows that the corresponding relative Haefliger-Wu or van Kampen obstruction to this extension vanishes. This is so because the Haefliger-Wu obstruction or the van Kampen obstruction involves 2 -fold products and double intersections but does not involve 3 -fold products and triple intersections.

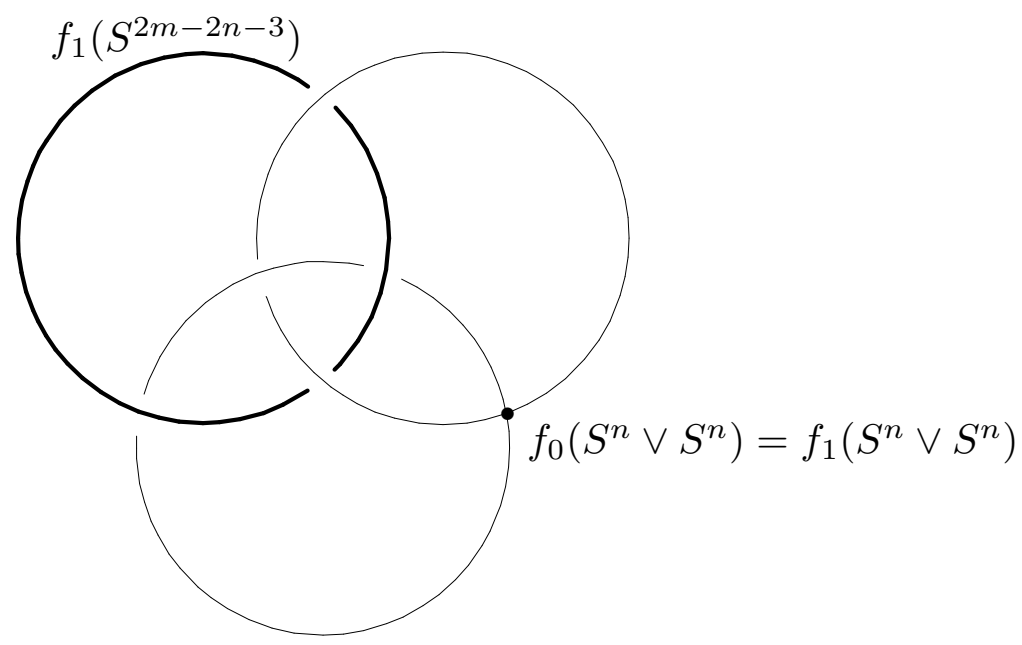

Figure 7.1

Proof of the Non-injectivity Example 5.7.d. The reader is recommended to read this proof first for $n=1$ and $m=3$. Let $N=S^{n} \vee S^{n} \sqcup S^{2 m-2 n-3}$. Take the standard embedding $f_{0}: N \rightarrow S^{m}$. Then $S^{m}-f_{0}\left(S^{n} \vee S^{n}\right) \simeq S^{m-n-1} \vee S^{m-n-1}$. Take a map $\varphi: S^{2 m-2 n-3} \rightarrow$ $S^{m}-f_{0}\left(S^{n} \vee S^{n}\right)$ representing the Whitehead product (for $m-n=2$, a commutator) of generators. If $n=1$ and $m=3$, then $\varphi$ is homotopic to an embedding by general position. If $n>1$, then $2 m \leq 3 n+3$ implies that $m \leq 2 n$, i.e. $m-(2 m-2 n-3) \geq 3$. Since also $2(2 m-2 n-3)-m+1 \leq m-n-2$ by the Penrose-Whitehead-Zeeman-Irwin Embedding Theorem 2.6.c, it follows that $\varphi$ is homotopic to an embedding. Define $f_{1}: N \rightarrow S^{m}$ on $S^{n} \vee S^{n}$ as $f_{0}$ and on $S^{2 m-2 n-3}$ as such an embedding (Figure 7.1). Since the homotopy class of $\varphi$ is non-trivial, it follows that $f_{1}: N \rightarrow S^{m}$ is not isotopic to the standard embedding $f_{0}: N \rightarrow S^{m}$.

Let us prove that $\alpha\left(f_{0}\right)=\alpha\left(f_{1}\right)$. Using 'finger moves' (analogously to the construction of Example 7.1 below) we construct a map $F: N \times I \rightarrow \mathbb{R}^{m} \times I$ such that

$F(x, 0)=\left(f_{0}(x), 0\right), \quad F(x, 1)=\left(f_{1}(x), 1\right) \quad$ and $\quad F\left(\left(S^{n} \vee S^{n}\right) \times I\right) \cap F\left(S^{2 m-2 n-3} \times I\right)=\emptyset$

Then there is a triangulation $T$ of $N$ such that no images of disjoint simplices intersect throughout $F_{t}$. Then the map $\widetilde{F}_{t}$ is well-defined on the simplicial deleted product $\widetilde{T}$. Since $\widetilde{T}$ is an equivariant deformation retract of $\widetilde{N}$, it follows that $\alpha\left(f_{0}\right)=\alpha\left(f_{1}\right)$. 
(One can also check that in general $\alpha_{G}^{m}(N) f_{0}=\alpha_{G}^{m}(N) f_{1}$ for each $G$, so $\alpha_{G}^{m}(N)$ is not injective.)
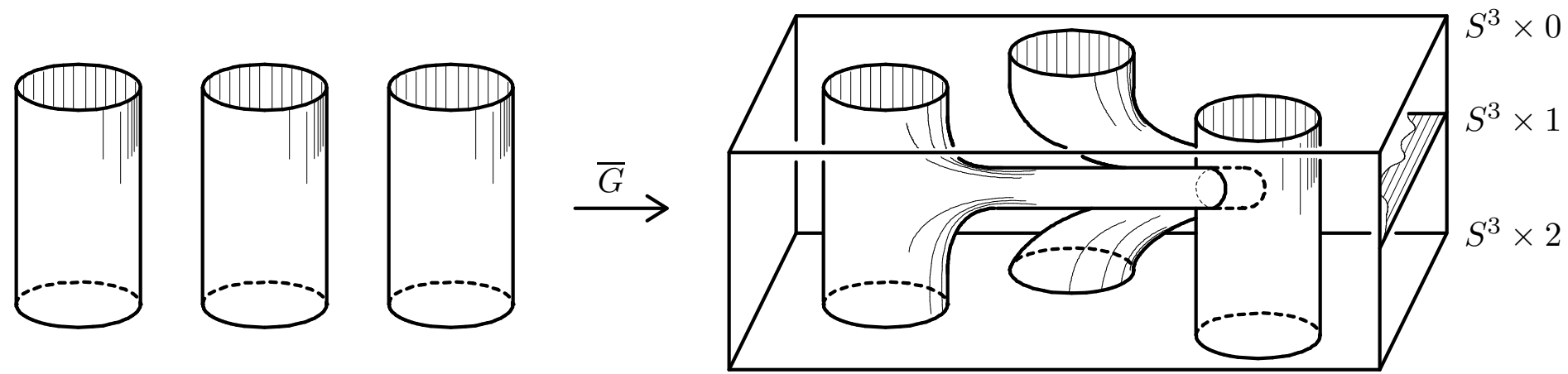

Figure 7.3

The above gives us a 3-dimensional visualization of the celebrated Casson finger moves. Combine the homotopy $F$ constructed in the above proof for $n=1$ and $m=3$ with the 'reverse' homotopy. We get a homotopy $G: N \times I \rightarrow \mathbb{R}^{3} \times I$ between standard embeddings $N \rightarrow \mathbb{R}^{3}$ (Figure 7.3). This homotopy is obtained from the identity isotopy by Casson finger moves.

We conjecture that the non-trivial embedding $f$ of the Non-injectivity Example 5.7.b can be obtained from explicitly defined Borromean rings $S^{n} \sqcup S^{n} \sqcup S^{2 m-2 n-3} \subset \mathbb{R}^{m}$ [Ha62', Ma90, Proposition 8.3] by 'wedging' $S^{n} \sqcup S^{n}$. We also conjecture that by joining the two $n$-spheres of the above linking by a tube we obtain a non-trivial embedding $S^{n} \sqcup S^{2 m-2 n-3} \rightarrow \mathbb{R}^{m}$ with trivial $\alpha$-invariant (although this is harder to prove: either we assume that $m-n \notin\{2,4,8\}$ and need to check that the linking coefficient of the obtained link is $\left[\iota_{m-n-1}, \iota_{m-n-1}\right] \neq 0$, or we need to apply the $\beta$-invariant [cf. Ha62', $\S 3$, $\mathrm{Sk}]$ ).

Example 7.1. There exist a 2-polyhedron $N$ non-embeddable into $\mathbb{R}^{4}$ but for which there exist a PL non-degenerate almost-embedding $f: N \rightarrow \mathbb{R}^{4}$.

The definition of a non-degenerate almost embedding is given before Lemma 4.2. The polyhedron $N$ from Example 7.1 is even topologically non-embeddable into $\mathbb{R}^{4}$.

Before constructing Example 7.1 let us explain its meaning. By the definition of the van Kampen obstruction (§4), Example 7.1 implies the Freedman-Krushkal-Teichner example, i.e. Theorem 4.1 for $n=2$. Take a triangulation $T$ of $N$ from the definition of an almost embedding $f: N \rightarrow \mathbb{R}^{4}$. Then the Gauss map $\widetilde{f}: \widetilde{T} \rightarrow S^{3}$ is well-defined on $\widetilde{T}$. Since $\widetilde{T}$ is an equivariant deformation retract of $\widetilde{N}$, it follows that there exists an equivariant mapping $\widetilde{N} \rightarrow S^{3}$. So Example 7.1 implies the Non-Embeddability Example 5.9.c for $m=2 n=4$. But Example 7.1 gives even more and shows the non-surjectivity not only of the Haefliger-Wu invariant, but also of the generalized Haefliger-Wu invariants (§5).

Preliminary construction for Example 7.1. Let $Q$ be the 2 -skeleton of the 6 -simplex minus the interior of one 2-simplex from this 2-skeleton. Recall from the subsection 'Ramsay link theory' of $\S 4$ that

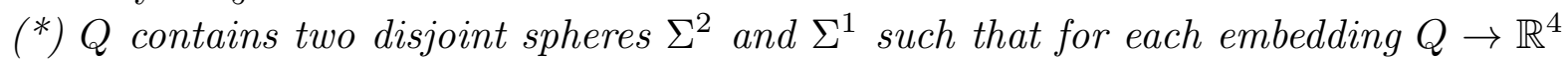
these spheres link with an odd linking number. 
An alternative proof of this fact is presented in the proof of the Linking Lemma 7.2 below.

In this section $\bar{Q}$ denotes a copy of the space $Q$ (for a subset $A \subset Q$ its copy is denoted by $\bar{A} \subset \bar{Q})$. Embed $Q \sqcup \bar{Q}$ into $\mathbb{R}^{4}$ in the standard way, i.e. so that

(a) the copies $Q$ and $\bar{Q}$ are far away from one another;

(b) both $\Sigma^{2}$ and $\bar{\Sigma}^{2}$ are unknotted.

Then $\Sigma^{2}$ and $\bar{\Sigma}^{2}$ are unlinked. Take any point $x \in \Sigma^{1}$. Join the points $x$ and $\bar{x}$ by an arc in $\mathbb{R}^{4}$ and pull small neighborhoods in $Q$ and $\bar{Q}$ of these points to each other along this arc. We obtain an embedding $Q \vee \bar{Q} \subset \mathbb{R}^{4}$ (Figure 7.4).

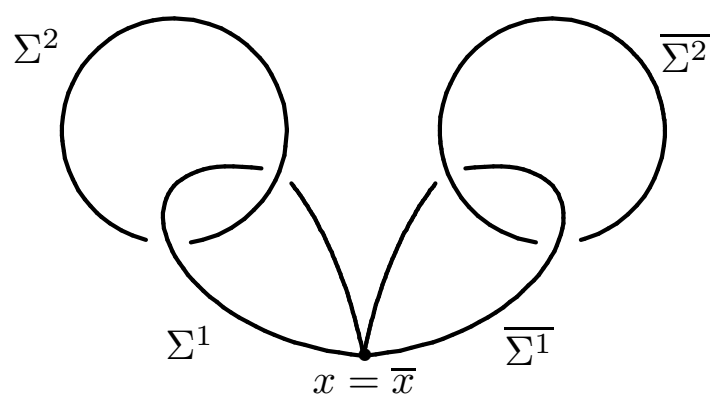

Figure 7.4

In Figure 7.4 each sphere $\Sigma^{1}, \bar{\Sigma}^{1}, \Sigma^{2}$ and $\bar{\Sigma}^{2}$ of dimensions $1,1,2$, and 2, respectively, is shown as 1-dimensional. Consider the wedge $\Sigma^{1} \vee \bar{\Sigma}^{1}$ with the base point $x=\bar{x}$. Then the inclusion $\Sigma^{1} \vee \bar{\Sigma}^{1} \subset \mathbb{R}^{4}-\left(\Sigma^{2} \sqcup \bar{\Sigma}^{2}\right)$ induces an isomorphism of the fundamental groups. Take generators $a$ and $\bar{a}$ of the group $\pi_{1}\left(\Sigma^{1} \vee \bar{\Sigma}^{1}\right)$ represented by the two (arbitrarily oriented) circles of the Figure Eight.

Sketch of the Freedman-Krushkal-Teichner construction. Here we sketch a construction of an example for Theorem 4.1 for $n=2$. This construction is a bit simpler than that of Example 7.1, but it makes vanishing of the obstruction less clear, and it gives neither Example 7.1 nor the Non-Embeddability Example 5.9.c.

Take a map $S^{1} \rightarrow \Sigma^{1} \vee \bar{\Sigma}^{1}$ representing the element $[a, \bar{a}]=a \bar{a} a^{-1} \bar{a}^{-1}$. Let $N^{\prime}$ be the mapping cone of the composition of this map with the inclusion $\Sigma^{1} \vee \bar{\Sigma}^{1} \subset Q \vee \bar{Q}$, i.e.

$$
N^{\prime}=B^{2} \bigcup_{[a, \bar{a}]: \partial B^{2} \rightarrow \Sigma^{1} \vee \bar{\Sigma}^{1}}(Q \vee \bar{Q})
$$

Let us sketch the proof of the nonembeddability of $N^{\prime}$ into $\mathbb{R}^{4}$. Suppose to the contrary that there exists an embedding $h: N^{\prime} \rightarrow \mathbb{R}^{4}$. The non-trivial element $[a, \bar{a}]$ of $\pi_{1}\left(\Sigma^{1} \vee \bar{\Sigma}^{1}\right)$ goes under $h$ to a loop in $\mathbb{R}^{4}-h\left(\Sigma^{2} \sqcup \bar{\Sigma}^{2}\right)$, which extends to $h D^{2}$ and hence is nullhomotopic. This is a contradiction because

$h$ induces a monomorphism $\pi_{1}\left(\Sigma^{1} \vee \bar{\Sigma}^{1}\right) \rightarrow \pi_{1}\left(\mathbb{R}^{4}-h\left(\Sigma^{2} \sqcup \bar{\Sigma}^{2}\right)\right)$.

If both $h \Sigma^{2}$ and $h \bar{\Sigma}^{2}$ are unknotted in $\mathbb{R}^{4}$, then the above assertion is clear. In general (i.e. when the spheres are knotted) the above assertion is proved using the Stallings theorem on the lower central series of groups [St65, FKT94], cf. below.

We have $V\left(N^{\prime}\right)=0$ because the van Kampen obstruction can only detects the homology property that the loop $[a, \bar{a}]$ is null-homologous (for a detailed proof see [FKT94]). 
Since the van Kampen obstruction is a complete obstruction for the existence of an equivariant map $\widetilde{N^{\prime}} \rightarrow S^{3}$ (for these dimensions), we obtain Non-embeddability Example 5.9.c for $m=2 n=4$.

Construction of Example 7.1. Take an embedding $Q \sqcup \bar{Q} \subset \mathbb{R}^{4}$ with the properties (a) and (b) from the Preliminary Construction. Take any points $x \in \Sigma^{1}$ and $y \in \Sigma^{2}$. Join points $x$ to $\bar{x}$ and $y$ to $\bar{y}$ by two arcs in $\mathbb{R}^{4}$. Pull small neighborhoods in $Q$ and $\bar{Q}$ of these points to each other along this arc (Figure 7.5). We obtain an embedding $K:=Q \underset{x=\bar{x}, y=\bar{y}}{\bigcup} \bar{Q} \subset \mathbb{R}^{4}$.

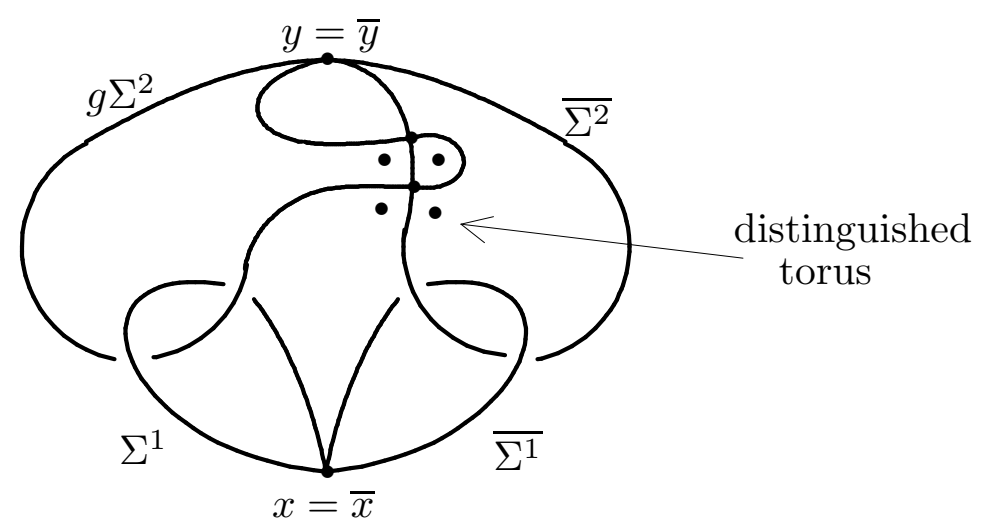

Figure 7.5

Push an 2-dimensional finger from a small disk $D^{2} \subset \Sigma^{2}$ near $y=\bar{y}$ intersecting $\bar{\Sigma}^{2}$ near $y=\bar{y}$. We get a new PL map $g: K \rightarrow \mathbb{R}^{4}$ which has transversal self-intersection points (Figure 7.5).

We can represent a disk neighborhood $B^{4}$ of an arbitrary intersection point $c \in \mathbb{R}^{4}$ as the product $B^{2} \times B^{2}$ of balls, where $0 \times 0$ corresponds to the intersection while $B^{2} \times 0$ and $0 \times B^{2}$ correspond to the images of $\Sigma^{2}$ and $\bar{\Sigma}^{2}$ (we denote by 0 the center of $B^{2}$ ). In a neighborhood of the point $c$ we have the distinguished or characteristic torus $\partial B^{2} \times \partial B^{2}$ [cf. Ca86, Ki89, FQ90]. In Figure 7.5 the 2-dimensional distinguished torus is shown as 0 -dimensional. By (b) we have $\pi_{1}\left(\mathbb{R}^{4}-\Sigma^{2} \vee \bar{\Sigma}^{2}\right) \cong \pi_{1}\left(S^{1} \vee S^{1}\right)$. Denote by $a$ and $\bar{a}$ the elements of this group represented by homeomorphisms $S^{1} \rightarrow z \vee S^{1}$ and $S^{1} \rightarrow S^{1} \vee z$ (for some point $z \in S^{1}$ ), respectively (with some orientations). With appropriate orientations the inclusions of $\partial B^{2} \times z$ and $z \times \partial B^{2}$ into $\mathbb{R}^{4}-\Sigma^{2} \vee \bar{\Sigma}^{2}$ are homotopic to $a$ and $\bar{a}$, respectively. Since the map

$$
a \bar{a} a^{-1} \bar{a}^{-1}: S^{1} \rightarrow S^{1} \vee S^{1} \cong\left(z \times \partial B^{2}\right) \vee\left(\partial B^{2} \times z\right)
$$

extends to a map $B^{2} \rightarrow \partial B^{2} \times \partial B^{2}$, it follows that $a \bar{a} a^{-1} \bar{a}^{-1}$ is null-homotopic in $\mathbb{R}^{4}-$ $g\left(\Sigma^{2} \vee \bar{\Sigma}^{2}\right)$. Then there exists a PL map $r: B^{2} \rightarrow \mathbb{R}^{4}-g\left(\Sigma^{2} \vee \bar{\Sigma}^{2}\right)$ such that $\left.r\right|_{\partial B^{2}}: \partial B^{2} \rightarrow$ $\Sigma^{1} \vee \bar{\Sigma}^{1}$ represents the commutator of the inclusions $\Sigma^{1} \subset \Sigma^{1} \vee \bar{\Sigma}^{1}$ and $\bar{\Sigma}^{1} \subset \Sigma^{1} \vee \bar{\Sigma}^{1}$. Roughly speaking, $r\left(B^{2}\right)$ is a torus $0 \times \partial B^{2} \times \partial B^{2}$. Set

$$
N=B^{2} \bigcup_{\partial B^{2}=\partial D^{2}}\left(K-\stackrel{\circ}{D}^{2}\right) \cup r\left(B^{2}\right) .
$$

Analogously to the proof of the Freedman-Krushkal-Teichner example above, $N$ does not embed into $\mathbb{R}^{4}$ (the details are analogous to Construction of Non-Embeddability Example 5.9.c below). 
We have $N \supset\left(K-\stackrel{\circ}{D}^{2}\right) \cup B^{2} \cong K$. Define a map $f: N \rightarrow \mathbb{R}^{4}$ on $\left(K-\stackrel{\circ}{D}^{2}\right) \cup B^{2}$ as the composition of a homeomorphism with $K$ and $g$, and on $r\left(B^{2}\right)$ as the identity. Then $\Sigma(f) \subset B^{2} \cup \bar{D}^{2}$. By the construction of the balls $D^{2}$ and $\bar{D}^{2}$ it follows that the balls $B^{2}$ and $\bar{D}^{2}$ are contained in the interiors of some adjacent 2-simplices of some triangulation $T$ of $N$. Hence $f$ is a non-degenerate almost embedding (whose image is $g(K) \cup r\left(B^{2}\right)$ ).

Construction of the Non-Embeddability Example 5.9.c. The case $m=3$ is proved in [GS06] using different ideas. The case $4 \leq m \leq n+1$ can either be proved analogously to [GS06] or is covered by the case $4 \leq m \geq n+2$ of the Non-Embeddability Example 5.9.c. So we present the proof for $m \geq n+2$. This is a higher-dimensional generalization of Example 7.1.

Let $l=m-n-1 \geq 1$. Denote by $\Delta_{a_{0} \ldots a_{s}}^{k}$ the $k$-skeleton of the $s$-simplex with vertices $a_{0} \ldots a_{s}$.

(The definition of $\Delta_{012 \ldots m+2}^{n}$ makes sense even for $n=l+1$, which case is outside the dimension range of the Non-Embeddability Example 5.9.c. If $n=l+1=1$, then $\Delta_{012 \ldots m+2}^{n}$ is one of the Kuratowski non-planar graphs, namely $K_{5}$, and if $n=l+1>1$ then $\Delta_{012 \ldots m+2}^{n}$ is an $n$-dimensional polyhedron non-embeddable in $\mathbb{R}^{2 n}$.)

Set

$$
\begin{gathered}
Q=\Delta_{12 \ldots m+2}^{n} \cup \operatorname{Con}\left(\Delta_{12 \ldots m+2}^{l}-\operatorname{Int} \Delta_{12 \ldots l+1}^{l}, 0\right), \quad K=Q \underset{0=\overline{0}, m=\bar{m}}{\bigcup} \bar{Q}, \\
\Sigma^{l}=\partial \Delta_{01 \ldots l+1}^{l+1} \quad \text { and } \quad \Sigma^{n}=\partial \Delta_{l+2 \ldots m+2}^{n+1} .
\end{gathered}
$$

The polyhedron $Q$ embeds in $\mathbb{R}^{m}$ (this was actually proved in the first two paragraphs of the proof of [SS92, Lemma 1.1]). Embed into $\mathbb{R}^{m}$ two copies of $Q$ which are far apart. Since $m \geq n+2$, we can join two points of $\Sigma^{n}$ and $\bar{\Sigma}^{n}$ by an arc and pull the points of the spheres together along this arc. Making the same construction for $\Sigma^{l}$ and $\bar{\Sigma}^{l}$ we obtain an embedding $K \rightarrow \mathbb{R}^{m}$; so we assume that $K$ is a subset of $\mathbb{R}^{m}$. We may assume that the wedge $\Sigma^{n} \vee \bar{\Sigma}^{n}$ is unknotted in $\mathbb{R}^{m}$. (If $m>n+2$, then this holds for any embedding $K \subset \mathbb{R}^{m}$ [Li65, Theorem 8]; if $m=n+2$, then for our embedding $Q \rightarrow \mathbb{R}^{m}$ the sphere $\Sigma^{n}$ is unknotted in $\mathbb{R}^{m}$, and we can choose an embedding $K \rightarrow \mathbb{R}^{m}$ so that $\Sigma^{n} \vee \bar{\Sigma}^{n}$ is unknotted in $\mathbb{R}^{m}$.)

Take a triangulation $T$ of $K$. Let $D^{n} \subset \Sigma^{n}$ and $\bar{D}^{n} \subset \bar{\Sigma}^{n}$ be PL disks each in the interiors of those $n$-simplices of $T$ that contain the common point $m=\bar{m}$ of $\Sigma^{n}$ and $\bar{\Sigma}^{n}$. Take points $a \in \stackrel{\circ}{D}^{n}, \bar{a} \in \check{D}^{n}$ and a small arc $s \subset \mathbb{R}^{m}$ joining $a$ to $\bar{a}$. By general position $s \cap K=\{a, \bar{a}\}$. Construct a new embedding $g: D^{n} \rightarrow \mathbb{R}^{m}$ obtained from the old one by pushing an $n$-dimensional finger from $D^{n}$ along the arc $s$. Let $\left.g\right|_{K-D^{n}}$ be the inclusion. We get a new PL map $g: K \rightarrow \mathbb{R}^{m}$ such that $\left.g\right|_{K-\check{D}^{n}}$ is an embedding but $g\left(D^{n}\right) \cap g\left(\bar{D}^{n}\right) \neq \emptyset$ (Figure 7.1.b).

By general position $\operatorname{dim}\left(g\left(D^{n}\right) \cap \bar{D}^{n}\right) \leq 2 n-m$ and $g\left(D^{n}\right)$ intersects $\bar{D}^{n}$ transversally. Denote by 0 the center of $B^{k}$. We can represent a disk neighborhood $B^{m}$ of an arbitrary point $c$ of this intersection as the product $B^{2 n-m} \times B^{l+1} \times B^{l+1}$ of balls, where $B^{2 n-m} \times 0 \times 0$ corresponds to the intersection, while

$$
B^{2 n-m} \times B^{l+1} \times 0 \text { and } B^{2 n-m} \times 0 \times B^{l+1}
$$

correspond to $g\left(D^{n}\right)$ and $\widetilde{D}^{n}$, respectively. In a neighborhood of the point $c$ we have the distinguished or characteristic torus $0 \times \partial B^{l+1} \times \partial B^{l+1}$. 
Since $\Sigma^{n} \vee \bar{\Sigma}^{n}$ is unknotted in $\mathbb{R}^{m} \subset S^{m}$, it follows that $\pi_{l}\left(\mathbb{R}^{m}-\Sigma^{n} \vee \bar{\Sigma}^{n}\right) \cong \pi_{l}\left(S^{l} \vee S^{l}\right)$. Denote by $\alpha$ and $\bar{\alpha}$ the elements of this group represented by the inclusions of components of the wedge (with some orientations). Take a point $y \in \partial B^{l+1}$. With appropriate orientations the inclusions of

$$
0 \times \partial B^{l+1} \times y \quad \text { and } \quad 0 \times y \times \partial B^{l+1} \quad \text { into } \quad \mathbb{R}^{m}-\Sigma^{n} \vee \bar{\Sigma}^{n}
$$

are homotopic to $\alpha$ and $\bar{\alpha}$, respectively. Since the Whitehead product

$$
[\alpha, \bar{\alpha}]: S^{2 l-1} \rightarrow S^{l} \vee S^{l} \cong\left(0 \times y \times \partial B^{l+1}\right) \vee\left(0 \times \partial B^{l+1} \times y\right)
$$

extends to a map $B^{2 l} \rightarrow 0 \times \partial B^{l+1} \times \partial B^{l+1}$ [Ca86, Ki89, FQ90], it follows that $[\alpha, \bar{\alpha}]$ is null-homotopic in $\mathbb{R}^{m}-\Sigma^{n} \vee \bar{\Sigma}^{n}$.

Denote the linking coefficient by $\operatorname{link}(\cdot, \cdot)$ Let $p=\operatorname{link}\left(\Sigma^{l}, \Sigma^{n}\right)$ and $\bar{p}=\operatorname{link}\left(\bar{\Sigma}^{l}, \bar{\Sigma}^{n}\right)$. The inclusions of $\Sigma^{l}$ and $\bar{\Sigma}^{l}$ into $\mathbb{R}^{m}-\Sigma^{n} \vee \bar{\Sigma}^{n}$ represent the elements $p \alpha$ and $\bar{p} \bar{\alpha}$ of the group $\pi_{l}\left(\mathbb{R}^{m}-\Sigma^{n} \vee \bar{\Sigma}^{n}\right)$, respectively. Since

$$
[p \alpha, \bar{p} \bar{\alpha}]=p \bar{p}[\alpha, \bar{\alpha}]=0 \in \pi_{2 l-1}\left(\mathbb{R}^{m}-\Sigma^{n} \vee \bar{\Sigma}^{n}\right),
$$

it follows that the Whitehead product of the (arbitrarily oriented) inclusions of $\Sigma^{l}$ and $\bar{\Sigma}^{l}$ into $\mathbb{R}^{m}-\Sigma^{n} \vee \bar{\Sigma}^{n}$ is null-homotopic. Hence there exists a PL map $r: B^{2 l} \rightarrow \mathbb{R}^{m}-\Sigma^{n} \vee \bar{\Sigma}^{n}$ whose restriction to $\partial B^{2 l}$ represents this Whitehead product. Set

$$
N=B^{n} \bigcup_{\partial B^{n}=\partial D^{n}}\left(K-\stackrel{\circ}{D}^{n}\right) \cup r\left(B^{2 l}\right) .
$$

Since $m \leq \frac{3 n}{2}+1$, it follows that $2 l \leq n$ and hence $\operatorname{dim} N=n$. Analogously to the proof of Example 7.1, there exists an almost embedding $N \rightarrow \mathbb{R}^{m}$. So it remains to prove that $N$ does not embed into $\mathbb{R}^{m}$.

Proof of the PL non-embeddability of $N$ into $\mathbb{R}^{m}$. Suppose to the contrary that there is a PL embedding $h: N \rightarrow S^{m}$. Let

$$
\Sigma_{1}^{n}=\left(\Sigma^{n}-\check{D}^{n}\right) \bigcup_{\partial B^{n}=\partial D^{n}} B^{n} \subset N .
$$

The map $\left.h \circ r\right|_{\partial B^{2 l}}$ can be extended to map

$$
h \circ r: B^{2 l} \rightarrow \mathbb{R}^{m}-h\left(\Sigma_{1}^{n} \vee \bar{\Sigma}^{n}\right) .
$$

Hence $\left.h \circ r\right|_{\partial B^{2 l}}$ is homotopically trivial in $\mathbb{R}^{m}-h\left(\Sigma_{1}^{n} \vee \bar{\Sigma}^{n}\right)$. Now we shall show the contrary and get a contradiction. Let

$$
q=\operatorname{link}\left(h \Sigma^{l}, h \Sigma_{1}^{n}\right) \quad \text { and } \quad \bar{q}=\operatorname{link}\left(h \bar{\Sigma}^{l}, h \bar{\Sigma}^{n}\right) .
$$

In the case $m>n+2$ by [Li65, Theorem 8] (cf. the construction of $N$ above) we have $S^{m}-h\left(\Sigma_{1}^{n} \vee \bar{\Sigma}^{n}\right) \simeq S^{l} \vee S^{l}$. Denote by $\beta$ and $\bar{\beta}$ the elements of the group $\pi_{l}\left(\mathbb{R}^{m}-h\left(\Sigma_{1}^{n} \vee\right.\right.$ 
$\left.\bar{\Sigma}^{n}\right)$ ) represented by the homeomorphisms $S^{l} \rightarrow y \vee S^{l}$ and $S^{l} \rightarrow S^{l} \vee y$ (where $y \in S^{l}$ ), respectively (with chosen orientations). Hence the homotopy class of the map

$$
\left.h \circ r\right|_{\partial B^{2 l}}: \partial B^{2 l} \rightarrow \mathbb{R}^{m}-h\left(\Sigma_{1}^{n} \vee \bar{\Sigma}^{n}\right) \quad \text { is } \quad q \bar{q}[\beta, \bar{\beta}] \in \pi_{2 l-1}\left(\mathbb{R}^{m}-h\left(\Sigma_{1}^{n} \vee \bar{\Sigma}^{n}\right)\right) .
$$

By the Hilton theorem [Po85, complement to Lectures 5 and 6, pp. 231, 257, or Hu59, p. 511], the map

$$
\varphi: \pi_{2 l-1}\left(S^{2 l-1}\right) \rightarrow \pi_{2 l-1}\left(S^{l} \vee S^{l}\right) \quad \text { defined by } \quad \varphi(\gamma)=[\beta, \bar{\beta}] \circ \gamma
$$

is injective (this can also be proved by using the homotopy exact sequence [Hu59, V.3]). Hence $[\beta, \bar{\beta}]$ has infinite order. This implies that the element $q \bar{q}[\beta, \bar{\beta}]$ is non-trivial because both $q$ and $\bar{q}$ are nonzero analogously to the property $\left({ }^{*}\right)$ in the preliminary construction for Example 7.1 (see the Linking Lemma 7.2 below).

In the case $m=n+2$ we have $l=1$. Consider the compositions

$$
\Sigma^{1} \subset \Sigma^{1} \vee \bar{\Sigma}^{1} \rightarrow \mathbb{R}^{n+2}-h\left(\Sigma^{n} \vee \bar{\Sigma}^{n}\right) \quad \text { and } \quad \bar{\Sigma}^{1} \subset \Sigma^{1} \vee \bar{\Sigma}^{1} \rightarrow \mathbb{R}^{n+2}-h\left(\Sigma^{n} \vee \bar{\Sigma}^{n}\right) .
$$

They are homologous to $q \beta$ and $\bar{q} \bar{\beta}$, respectively. The commutator of the homotopy classes of the above compositions is non-zero because the inclusion $\Sigma^{1} \vee \bar{\Sigma}^{1} \subset \mathbb{R}^{n+2}-\left(\Sigma^{n} \vee \bar{\Sigma}^{n}\right)$ induces a monomorphism of the fundamental groups. The latter is proved analogously to [FKT94, proof of Lemmas 7 and 8] using the Stallings theorem [St65] and that by the Linking Lemma 7.2 below

$\operatorname{link}\left(\Sigma^{n}, \Sigma^{1}\right) \equiv \operatorname{link}\left(\bar{\Sigma}^{n}, \bar{\Sigma}^{1}\right) \equiv 1 \quad \bmod 2 \quad$ and $\quad \operatorname{link}\left(\Sigma^{n}, \bar{\Sigma}^{1}\right)=\operatorname{link}\left(\bar{\Sigma}^{n}, \Sigma^{1}\right)=0$.

The Linking Lemma 7.2. For any $P L$ embedding $K \subset \mathbb{R}^{m}$ of the above polyhedron $K$ the pairs $\left(\Sigma^{n}, \bar{\Sigma}^{l}\right)$ and $\left(\bar{\Sigma}^{n}, \Sigma^{l}\right)$ are unlinked, and $\operatorname{link}\left(\Sigma^{n}, \Sigma^{l}\right)$ is odd [cf. SS92, Lemma 1.4, FKT94, Lemmas 6, 7 and 8].

Sketch of the proof. The unlinking part follows because $\Sigma^{l}$ (resp. $\bar{\Sigma}^{l}$ ) bounds a disk $\Delta_{01 \ldots l+1}^{l+1}\left(\right.$ resp. $\left.\bar{\Delta}_{01 \ldots l+1}^{l+1}\right)$ in $K-\bar{\Sigma}^{n}$ (resp. in $K-\Sigma^{n}$ ).

We illustrate the idea of proof of the linking part by proving its particular case $m=$ $2 n=4 l=4$ (for which, however, there exists a simpler proof). Recall the formulation for this case:

Let $Q=\Delta_{01 \ldots 6}^{2}-\triangle_{012}^{2}$. Let $\Sigma^{1}=\partial \Delta_{012}^{2}$ and let $\Sigma^{2}$ be the union of 2-simplices disjoint from $\Delta_{012}^{2}$. Then for each embedding $Q \rightarrow \mathbb{R}^{4}$ these spheres link with an odd linking number.

First we prove a simpler result: that $\operatorname{link}\left(f \Sigma^{2}, f \Sigma^{1}\right) \neq 0$ for each embedding $f: Q \rightarrow$ $\mathbb{R}^{4}$. (The higher-dimensional analogue of this simpler result is sufficient for the proof of the non-embeddability for $m \geq n+3$.) If $\operatorname{link}\left(f \Sigma^{2}, f \Sigma^{1}\right)=0$, then $f \Sigma^{1}$ spans a 2-disk outside $f \Sigma^{2}$. Hence we can construct an almost embedding $\Delta_{01 \ldots 6}^{2} \rightarrow \mathbb{R}^{4}$. Therefore there is an equivariant map $\widetilde{\Delta_{01 \ldots 6}^{2}} \rightarrow S^{3}$. Then by [Ya54], any equivariant map $\widetilde{\Delta_{01 \ldots 6}^{2}} \rightarrow S^{4}$ should induce a trivial homomorphism in $H_{4}^{e q}\left(\cdot, \mathbb{Z}_{2}\right)$. But

there is an embedding $g: \Delta_{01 \ldots 6}^{2} \rightarrow \mathbb{R}^{5}$ such that $\widetilde{g}: \widetilde{\Delta_{01 \ldots .}^{2}} \rightarrow S^{4}$ induces a non-trivial homomorphism in $H_{4}^{e q}\left(\cdot, \mathbb{Z}_{2}\right)$. 
Indeed, $\Delta_{01 \ldots 6}^{2} \cong \Delta_{01 \ldots 5}^{2} \cup \operatorname{Con} \Delta_{01 \ldots 5}^{1}$, where $\Delta_{01 \ldots 5}^{5}$ is a regular 5-simplex inscribed into the standard unit 4 -sphere in $\mathbb{R}^{5}$ and the vertex of the cone is 0 . This homeomorphism defines an embedding $g: \Delta_{01 \ldots 6}^{2} \rightarrow \mathbb{R}^{5}$. The union of 4-cells in the simplicial deleted product $\widetilde{\Delta_{01 \ldots 6}^{2}}$ is a $\mathbb{Z}_{2}$-equivariant 4 -cycle. Let $p$ be a vertex of $\Delta_{01 \ldots 5}^{5}$. The map $\widetilde{g}$ maps a small neighborhood in $\widehat{\Delta_{01 \ldots 6}^{2}}$ of the point $(p, 0)$ homeomorphically onto a neighborhood in $S^{4}$ of $p$. Hence the $\widetilde{g}$-image of the above 4-cycle is non-trivial, and so $\widetilde{g}$ induces a non-trivial homomorphism in $H_{4}^{e q}\left(\cdot, \mathbb{Z}_{2}\right)$.

In order to prove the full strength of the particular case $m=2 n=4 l=4$, assume to the contrary that $\operatorname{link}\left(f \Sigma^{n}, f \Sigma^{l}\right)$ is even. Let $\Delta^{\prime}$ be a polyhedron obtained from $\Delta_{012}^{2}$ by removing the interiors of an even number $2 r$ of disjoint 2-disks in $\Delta_{012}^{2}$ and running $r$ pairwise disjoint tubes between the holes thus formed. Then $f \Sigma^{1}$ spans $\Delta^{\prime}$ outside $f \Sigma^{2}$. Let $X=Q \underset{\partial \Delta_{012}^{2}=\partial \Delta^{\prime}}{\bigcup} \Delta^{\prime}$. Then there is an equivariant map $\widetilde{X} \rightarrow S^{3}$. Therefore by [Ya54] any equivariant map $\widetilde{X} \rightarrow S^{4}$ should induce a trivial homomorphism in $H_{4}^{e q}\left(\cdot, \mathbb{Z}_{2}\right)$.

Let $p: \Delta \rightarrow \Delta_{01 \ldots 6}^{2}$ be a map which is the identity on $Q$ and such that $p\left(\stackrel{\circ}{\Delta}^{\prime}\right)=\stackrel{\Delta}{\Delta}_{012}^{2}$. Then $\tilde{p}: \widetilde{X} \rightarrow \widehat{\Delta_{01 \ldots 6}^{2}}$ induces an epimorphism in $H_{4}^{e q}\left(\cdot, \mathbb{Z}_{2}\right)$ [SS92, p. 278]. Then $\tilde{f} \circ \widetilde{p}$ induces a non-trivial homomorphism in $H_{4}^{e q}$, which is a contradiction.

Proof of the TOP non-embeddability of $N$ into $\mathbb{R}^{m}$ (sketch). For $m \geq n+3$ by [Br72] TOP non-embeddability follows from PL non-embeddability. For $m=n+2$ we do the following. There are arbitrarily close approximations $h^{\prime}: N \rightarrow \mathbb{R}^{m}$ to the embedding $h$ by PL almost embeddings (for certain triangulations of $N$ ). By general position we may assume that $\left.h^{\prime}\right|_{\Sigma^{1}}$ and $\left.h^{\prime}\right|_{\bar{\Sigma}^{1}}$ are PL embeddings. The rest of the proof is analogous to the PL case (in which we need to replace $h$ by $h^{\prime}$ ), because we only used the Linking Lemma 7.2 but not the fact that $h$ is an embedding.

Appendix: Borromean rings and the Boy immersion. The following was proved in [Ak96", cf. Fr87, ARS02]. Let $h: \mathbb{R} P^{2} \rightarrow \mathbb{R}^{3}$ be the Boy immersion. Fix any orientation on the sphere $S^{2}$ and on the double points circle $\Delta(h)$. Take a small closed ball $D^{3} \subset \mathbb{R}^{3}$ containing the triple point of $h$. Let $r: S^{2} \rightarrow \mathbb{R} P^{2}$ be the standard double covering. Denote by $\pi_{1}: \mathbb{R}^{3} \times \mathbb{R} \rightarrow \mathbb{R}^{3}$ and $\pi_{2}: \mathbb{R}^{3} \times \mathbb{R} \rightarrow \mathbb{R}$ the projections. Take a general position smooth map $\bar{h}:\left(S^{2}-h_{1}^{-1} \stackrel{\circ}{ }^{3}\right) \rightarrow \mathbb{R}^{3} \times \mathbb{R}$ such that $\pi_{1} \circ \bar{h}=h \circ r$ and for each points $x, y \in S^{2}-(h \circ r)^{-1} D^{3}$ such that $h r x=h r y$ and $\pi_{2} \bar{h} x>\pi_{2} \bar{h} y$ the following three vectors form a positive basis of $\mathbb{R}^{3}$ at the point $h r x=h r y$ : the vector along the orientation of $\Delta$, the normal vector to the small sheet of $h\left(\mathbb{R} P^{2}\right)$ containing $x$ and the normal vector to the

small sheet of $h\left(\mathbb{R} P^{2}\right)$ containing $y$. Then $\left.\bar{h}\right|_{(h \circ r)-1} \partial D^{3} \rightarrow \partial D^{3} \times \mathbb{R}$ forms the Borromean rings linking (after the identification $\partial D^{3} \times \mathbb{R} \cong \mathbb{R}^{3}$ ).

\section{The DisjunCtion Method}

In this section we illustrate the disjunction method by sketching some ideas of the proof of the surjectivity in the Haefliger-Weber Theorem 5.4 for the PL case, and of Theorem 5.5. The first of these results can be restated as follows.

The Weber Theorem 8.1. [We67] Suppose that $N$ is an n-polyhedron, $2 m \geq 3 n+3$ and $\Phi: \widetilde{N} \rightarrow S^{m-1}$ is an equivariant map. Then there is a PL embedding

$$
f: N \rightarrow \mathbb{R}^{m} \text { such that } \tilde{f} \simeq_{e q} \Phi \text { on } \tilde{N} .
$$


Simplices of any triangulation $T$ are assumed to be linearly ordered with respect to increasing dimension. The lexicographical ordering on $T \times T$ is used.

Proof of the Weber Theorem 8.1 for $m=2 n+1$. We present the proof for $m=3$ and $n=1$ (the general case $m=2 n+1$ is proved analogously). Take a general position map $f: N \rightarrow \mathbb{R}^{3}$. Then it is an embedding. So we only need to modify it in order to obtain the property $\tilde{f} \simeq_{e q} \Phi$. This property does not follow by general position. We obtain it by applying the van Kampen finger moves, i.e. by winding edges of the graph $N$ around images of other edges. Note that van Kampen invented his finger moves for the proof of Lemma 4.2, and here we present a generalization of the van Kampen finger moves.

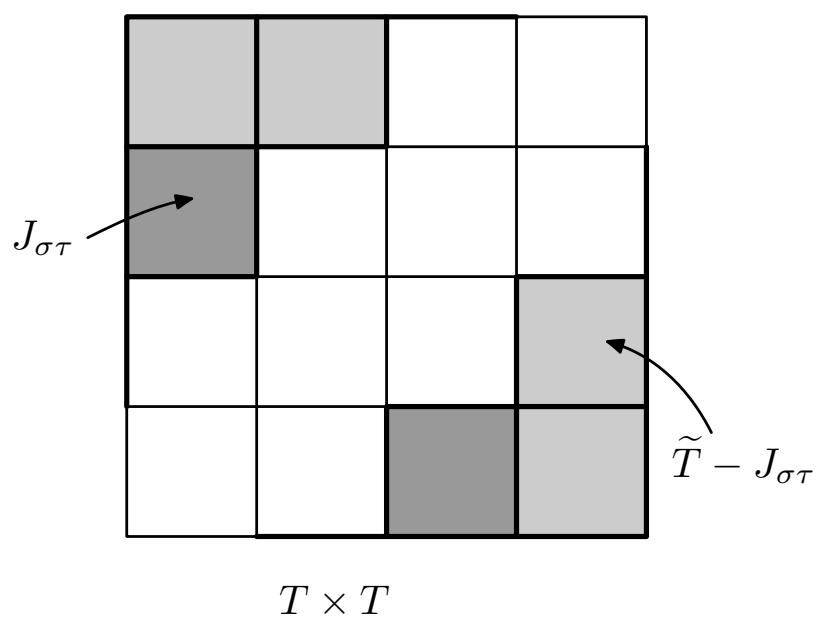

Figure 8.1

Proposition 8.2. Let $T$ be a triangulation of a 1-polyhedron $N$ (i.e. $T$ is a graph representing this 1-polyhedron). For each pair of edges $\sigma, \tau \in T$ such that $\sigma \leq \tau$ there exists a PL embedding

$$
f: N \rightarrow \mathbb{R}^{3} \quad \text { such that } \tilde{f} \simeq_{e q} \Phi \quad \text { on } \quad J_{\sigma \tau}=\bigcup_{\sigma \times \tau>\alpha \times \beta \in \widetilde{T}} \alpha \times \beta
$$

Theorem 8.1 for $m=2 n+1=3$ follows from Proposition 8.2 by taking $\sigma$ and $\tau$ to be the last simplices of $T$.

Proof of Proposition 8.2. 1st step: construction of balls. By induction on $\sigma \times \tau$. If both $\sigma$ and $\tau$ are the first edge of $T$, then $\operatorname{dim} J_{\sigma \tau}=1$, hence Proposition 8.2 is true by general position. Now suppose as inductive hypothesis that $\tilde{f} \simeq_{e q} \Phi$ on $J_{\sigma \tau}$ for an embedding $f: N \rightarrow \mathbb{R}^{3}$. We need to prove that for $\sigma \cap \tau=\emptyset$ there exists a map

$$
f^{+}: N \rightarrow \mathbb{R}^{3} \quad \text { such that } \quad \tilde{f}^{+} \simeq_{e q} \Phi \quad \text { on } \quad J_{\sigma \tau} \cup \sigma \times \tau \cup \tau \times \sigma .
$$

Take points $C_{\sigma} \in \stackrel{\circ}{\sigma}$ and $C_{\tau} \in \stackrel{\circ}{\tau}$ (Figure 8.2). Join their images by an arc

$$
C^{1} \subset \mathbb{R}^{3} \text { such that } C^{1} \cap f N=\left\{f C_{\sigma}, f C_{\tau}\right\} \text {. }
$$


Let $D^{3}$ be a small ball neighborhood of $C^{1}$ in $\mathbb{R}^{3}$ such that $f^{-1} D^{3}$ is disjoint union of arcs $D_{\sigma} \subset \stackrel{\circ}{\sigma}$ and $D_{\tau} \subset \stackrel{\circ}{\tau}$ containing points $C_{\sigma}$ and $C_{\tau}$, respectively. We may assume that $f D_{\sigma}$ is unknotted in $D^{3}$. Hence a homotopy equivalence $h: D^{3}-f D_{\sigma} \rightarrow S^{1}$ is constructed analogously to the homotopy equivalence $S^{m}-f S^{q} \rightarrow S^{m-q-1}$ from the definition of the linking coefficients at the beginning of $\S 3$.

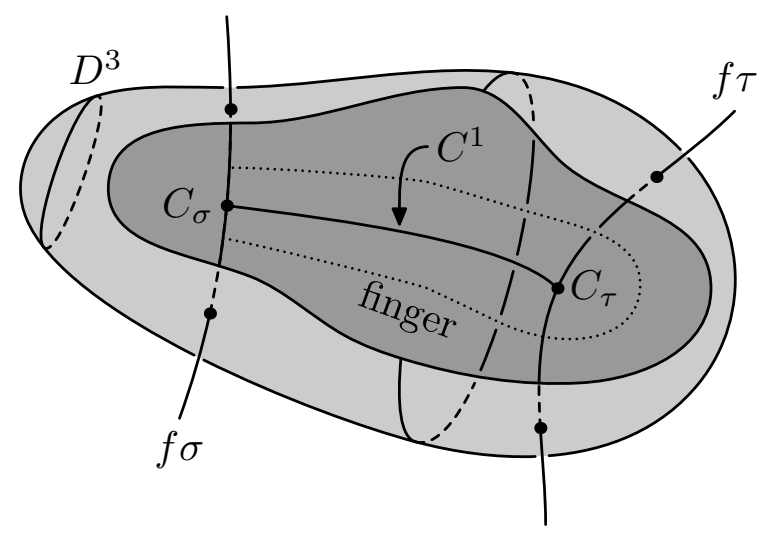

Figure 8.2

Proof of Proposition 8.2. 2nd step: the van Kampen finger move. In order to construct such an $f^{+}$we shall wind the arc $\left.f\right|_{D_{\tau}}$ around $f D_{\sigma}$ in $D^{3}-f D_{\sigma}$ (Figure 8.3).

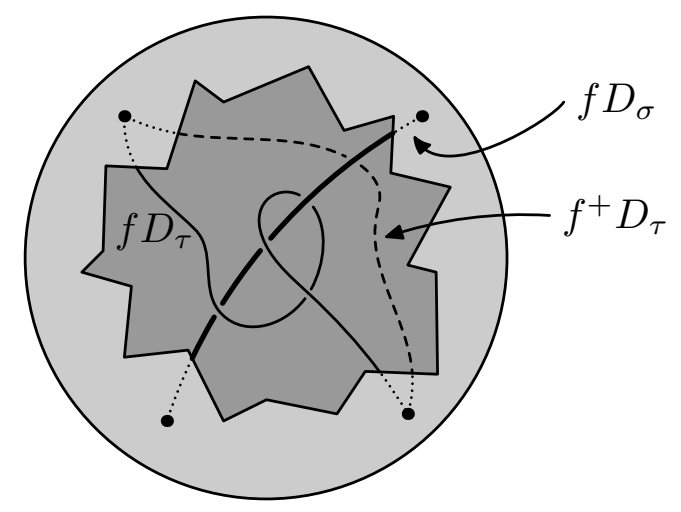

Figure 8.3

Take any embedding

$$
f^{+}: D_{\sigma} \sqcup D_{\tau} \rightarrow D^{3} \quad \text { such that } f^{+}=f \quad \text { on } \quad D_{\sigma} \sqcup \partial D_{\tau} .
$$

Let $D_{+}$be a copy of $D_{\tau}$. Identify $S^{1}$ with $D_{\tau} \bigcup_{\partial D_{\tau}=\partial D_{+}} D_{+}$. Define a map

$$
h_{f f^{+}}: S^{1} \rightarrow D^{3}-f D_{\sigma} \stackrel{h}{\rightarrow} S^{1} \quad \text { by } \quad h_{f f^{+}}(x)=\left\{\begin{array}{ll}
h(f(x)), & x \in D_{\tau} \\
h\left(f^{+}(x)\right), & x \in D_{+}
\end{array} .\right.
$$

Since $f^{+}=f$ on $D_{\sigma} \sqcup \partial D_{\tau}$, it follows that there is a homotopy $f_{t}: D_{\sigma} \sqcup D_{\tau} \rightarrow D^{3}$ from $f$ to $f^{+}$fixed on $D_{\sigma} \sqcup \partial D_{\tau}$. Denote by $\widetilde{f}, \Phi$ and $\widetilde{f^{+}}$the restrictions of these maps to $D_{\sigma} \times D_{\tau}$, and by $\widetilde{f}_{t}$ the restriction of this map to $\partial\left(D_{\sigma} \times D_{\tau}\right)$. Define a map

$$
H_{\tilde{f} \tilde{f}^{+}}: \partial\left(D_{\sigma} \times D_{\tau} \times I\right) \rightarrow S^{2} \text { by }
$$




$$
\left.H_{\widetilde{f} \tilde{f}^{+}}\right|_{D_{\sigma} \times D_{\tau} \times 0}=\tilde{f},\left.\quad H_{\widetilde{f} \tilde{f}^{+}}\right|_{D_{\sigma} \times D_{\tau} \times 1}=\widetilde{f}^{+},\left.\quad H_{\tilde{f} \tilde{f}^{+}}\right|_{\partial\left(D_{\sigma} \times D_{\tau}\right) \times I}=\tilde{f}_{t} .
$$

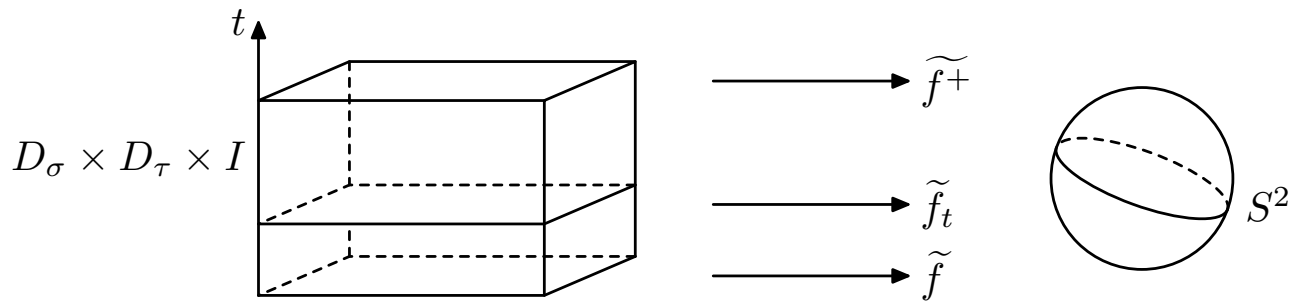

Figure 8.4

By [We67, Lemma 1] we have

$$
\left[H_{\widetilde{f} \tilde{f}^{+}}\right]=\Sigma\left[h_{f f^{+}}\right] \in \pi_{2}\left(S^{2}\right) .
$$

By the equivariant analogue of the Borsuk Homotopy Extension Theorem, there is an equivariant extension $\Psi: \widetilde{K} \rightarrow S^{2}$ of $\left.\widetilde{f}\right|_{J_{\sigma \tau} \cup\left(\sigma \times \tau-\dot{D}_{\sigma} \times \stackrel{D}{\tau}_{\tau}\right)}$ such that $\Psi \simeq_{e q} \Phi$. We may assume that $\Psi=\Phi$, so that $\Phi=\bar{f}$ on $\partial\left(D_{\sigma} \times D_{\tau}\right)$. Therefore $H_{\Phi \tilde{f}^{+}}$can be defined analogously to the above. Also we can define $H_{\Phi \tilde{f}}$ analogously to the above using the constant homotopy between $\Phi$ and $\tilde{f}$ on $\partial\left(D_{\sigma} \times D_{\tau}\right)$. Then

$$
\left[H_{\Phi \tilde{f}^{+}}\right]=\left[H_{\Phi \tilde{f}}\right]+\left[H_{\tilde{f} \tilde{f}^{+}}\right]=\left[H_{\Phi \tilde{f}}\right]+\Sigma\left[h_{f f^{+}}\right] \in \pi_{2}\left(S^{2}\right) .
$$

For every element $\beta \in \pi_{1}\left(S^{1}\right)$ there is an embedding $f^{+}: D_{\tau} \rightarrow D^{3}-f D_{\sigma}$ such that $\left[h_{f f^{+}}\right]=\beta$. Therefore by the Suspension Theorem, there exists a map $f^{+}: D_{\tau} \rightarrow$ $D^{3}-f D_{\sigma}$ such that $\left[H_{\Phi \tilde{f}^{+}}\right]=0$. Extend this $f^{+}$to all $N$ by $f$. Then $f^{+}$is as required.

Proof of the Weber Theorem $\mathbf{8 . 1}$ for $m=2 n \geq 6$. Let us introduce some natural and useful definitions. Fix a triangulation $T$ of an $n$-polyhedron $N$. A map $f: N \rightarrow \mathbb{R}^{m}$ is an embedding if and only if the following conditions hold:

$f$ is (T-)nondegenerate, i.e. $\left.f\right|_{\alpha}$ is an embedding for each $\alpha \in T$;

$f$ is a (T-)almost embedding, i.e. $f \alpha \cap f \beta=\emptyset$ for each $\alpha \times \beta \subset \widetilde{T}$;

$f$ is a $T$-immersion, i.e. $f \alpha \cap f \beta=f(\alpha \cap \beta)$ for each $\alpha, \beta \in T$ such that $\alpha \cap \beta \neq \emptyset$.

Plan of the proof of the Weber Theorem 8.1 for $m=2 n \geq 6$. Take a triangulation $T$ of $N$ and a general position map $f: N \rightarrow \mathbb{R}^{m}$ that is linear on simplices of $T$. Hence $f$ is nondegenerate. By general position, the properties of almost embedding and $T$-immersion hold unless $\operatorname{dim} \alpha=\operatorname{dim} \beta=n$.

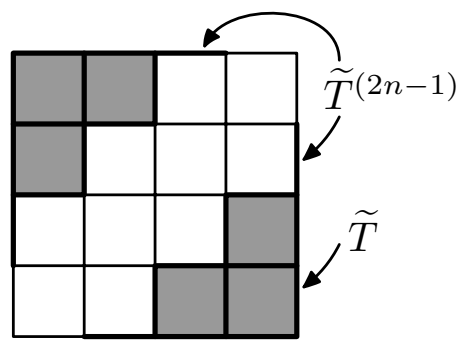

Figure 8.5 
Step 1. Wind $n$-simplices of $T$ around $(n-1)$-simplices (analogously to the case $m=$ $2 n+1)$ and thus modify $f$ to obtain additionally the condition $f \simeq_{e q} \Phi$ on the $(2 n-1)$ skeleton of $\widetilde{T}$ (Figure 8.5). Such a van Kampen finger move is a higher-dimensional generalization of the fifth Reidemeister move (Figure 4.2.V).

Step 2. Now it is possible to remove intersections of disjoint $n$-simplices of $T$ and thus modify $f$ so as to make it additionally an almost embedding. This construction is a higher-dimensional generalization of the first and the second Reidemeister moves (Figure 4.2.I and 4.2.II), which are called the Penrose-Whitehead-Zeeman trick and the Whitney trick (cf. §4), respectively. The details for Step 2 are given as Proposition 8.3 below.

Step 1 and Step 2 together are analogues of Lemma 4.2.

Step 3. Wind $n$-simplices of $T$ around $n$-simplices (analogously to the case $m=2 n+1$ ) and thus modify $f$ to obtain additionally the condition $f \simeq_{e q} \Phi$ on $\widetilde{T}$. These are the van Kampen finger moves in other dimension.

Step 4. Remove unnecessary intersections of $n$-simplices of $T$ having a common face and thus modify $f$ so as to make it additionally a $T$-immersion (and hence an embedding). This construction is a higher-dimensional generalization of the fourth Reidemeister move (Figure 4.2.IV), an analogue of the Freedman-Krushkal-Teichner Lemma 4.3, and is called the Freedman-Krushkal-Teichner trick.

Proposition 8.3. (cf. Proposition 8.2) Let $T$ be a triangulation of an n-polyhedron $N$. For each pair of $n$-simplices $\sigma, \tau \in T$ such that $\sigma<\tau$ there exists a nondegenerate $P L$ map

$$
f: N \rightarrow \mathbb{R}^{2 n} \quad \text { such that } f \alpha \cap f \beta=\emptyset \quad \text { if } \quad \sigma \times \tau>\alpha \times \beta \in \widetilde{T} .
$$
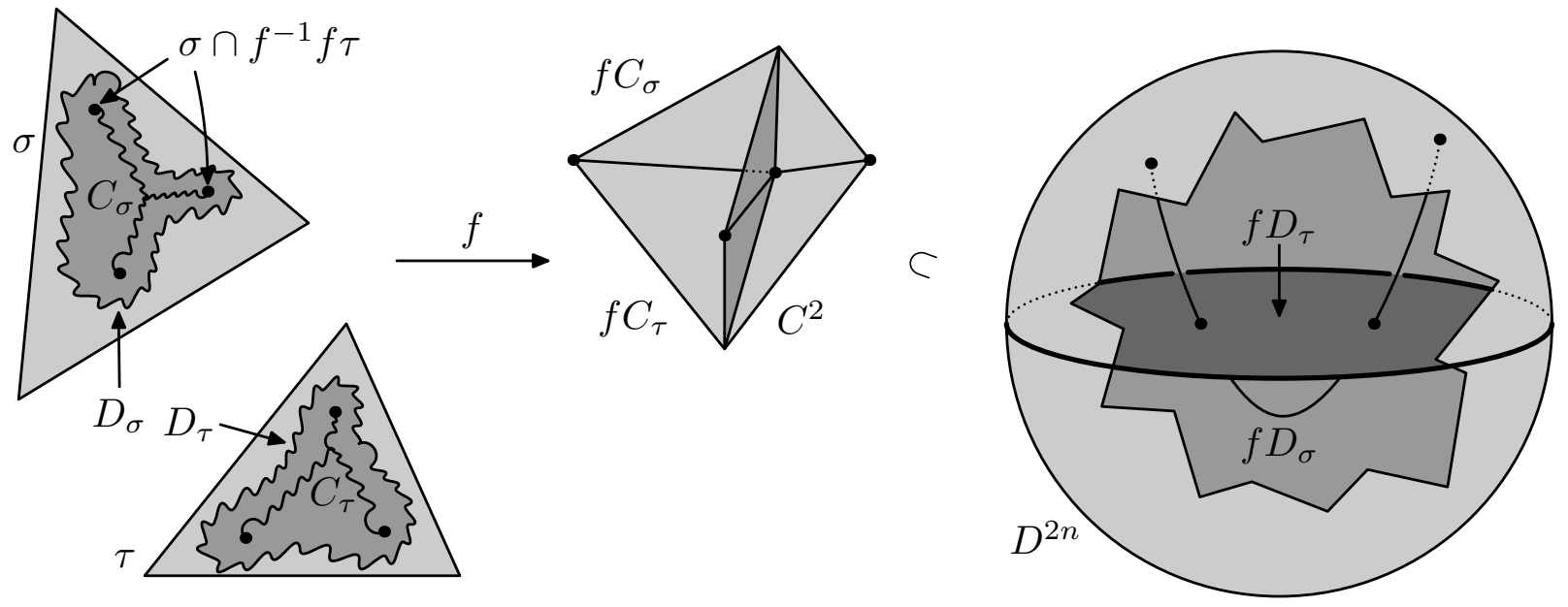

Figure 8.6

Proof. As inductive hypothesis, assume that we have such $f$ for a pair $\sigma \times \tau \subset \widetilde{T}$. We need to prove that there exists a map

$$
f^{+}: N \rightarrow \mathbb{R}^{2 n} \quad \text { such that } \quad f \alpha \cap f \beta=\emptyset \quad \text { if } \quad \sigma \times \tau \geq \alpha \times \beta \in \widetilde{T} .
$$

By the inductive hypothesis $f \sigma \cap f \tau=f \stackrel{\circ}{\sigma} \cap f \circ$ is a finite set of points. Let $C_{\sigma} \subset \stackrel{\circ}{\sigma}$ be an arc containing the points of $\sigma \cap f^{-1} \tau$ (Figure 8.6). Let $C_{\tau} \subset \stackrel{\sim}{\tau}$ be an arc containing the 
points of $\tau \cap f^{-1} \sigma$ 'in the same order' as $C_{\sigma}$. Let $C^{2} \subset \mathbb{R}^{2 n}$ be a union of disks such that $C^{2} \cap f \sigma=f C_{\sigma}$ and $C^{2} \cap f \tau=f C_{\tau}$.

Let $D^{2 n}$ be a small neighborhood of $C^{2}$ in $\mathbb{R}^{2 n}$ such that $f^{-1} D^{2 n}$ is disjoint union of $n$-balls $D_{\sigma} \subset \stackrel{\circ}{\sigma}$ and $D_{\tau} \subset \stackrel{\circ}{\tau}$, which are small neighborhoods of the $\operatorname{arcs} C_{\sigma}$ and $C_{\tau}$ in $N$. We have

$\left.f\right|_{D_{\sigma}}$ and $\left.f\right|_{D_{\tau}}$ are proper embeddings into $D^{2 n}$

$f \sigma \cap f \tau \subset \stackrel{\circ}{D}^{2 n}$

$D_{\sigma}=\sigma \cap f^{-1} D^{2 n}$ and $D_{\tau}=\tau \cap f^{-1} D^{2 n} ;$ and

$f \partial D_{\sigma} \cap f D_{\tau}=f \partial D_{\tau} \cap f D_{\sigma}=\emptyset$.

Since $n \geq 3$, it follows that a homotopy equivalence $h: D^{2 n}-f D_{\sigma} \rightarrow S^{n-1}$ is constructed analogously to the homotopy equivalence $S^{m}-f S^{q} \rightarrow S^{m-q-1}$ from the definition of the linking coefficients at the beginning of $\S 3$. The coefficient of the intersection of $f D_{\sigma}$ and $f D_{\tau}$ is the homotopy class

$$
I\left(f D_{\sigma}, f D_{\tau}\right)=\left[\left.f\right|_{\partial D_{\tau}}: \partial D_{\tau} \rightarrow D^{2 n}-f D_{\sigma} \stackrel{h}{\rightarrow} S^{n-1}\right] \in \pi_{n-1}\left(S^{n-1}\right) .
$$

We have

$$
\pm \Sigma^{n} I\left(f D_{\sigma}, f D_{\tau}\right)=\left[\left.\widetilde{f}\right|_{\partial\left(D_{\sigma} \times D_{\tau}\right)}\right]=\left[\left.\Phi\right|_{\partial\left(D_{\sigma} \times D_{\tau}\right)}\right]=0 \in \pi_{2 n-1}\left(S^{2 n-1}\right) .
$$

Here the first equality is [We67, Proposition 1], the second equality holds because $\tilde{f} \simeq \Phi$ on $\partial\left(D_{\sigma} \times D_{\tau}\right)$ by the inductive hypothesis, and the third equality holds since $\Phi$ is defined over $\widetilde{T} \supset D_{\sigma} \times D_{\tau}$. By the Freudenthal Suspension Theorem $I\left(f D_{\sigma}, f D_{\tau}\right)=0$. Hence the embedding $\left.f\right|_{\partial D_{\tau}}$ extends to a map $f^{+}: D_{\tau} \rightarrow D^{2 n}-f D_{\sigma}$. Using the PenroseWhitehead-Zeeman trick we modify $f^{\prime}$ to an embedding. Extend $f^{+}$over the entire $N$ by $f$. By general position $C^{2}$ (and hence $D^{2 n}$ and $f^{+} D_{\tau}$ ) is disjoint with $f(N-\operatorname{st} \sigma-\operatorname{st} \tau)$. Therefore $f^{+}$is as required.

Definition of a regular neighborhood. The notions of collapse and regular neighborhood are used in our proofs and are generally important in topology.

A polyhedron $Y$ is said to be obtained from a polyhedron $K$ by an elementary collapse, if $K=Y \cup B^{n}$ and $Y \cap B^{n}=B^{n-1}$, where $B^{n-1}$ is a face of the ball $B^{n}$. This elementary collapse is said to be made from $\mathrm{Cl}\left(\partial B^{n}-B^{n-1}\right)$ along $B^{n}$ to $B^{n-1}$. A polyhedron $K$ collapses to $Y$ (notation: $K \searrow Y$ ) if there exists a sequence of elementary collapses $K=K_{0} \searrow K_{1} \searrow \cdots \searrow K_{n}=Y$. A polyhedron $K$ is collapsible, if it collapses to a point.

Clearly, the ball $B^{n}$ is collapsible, since it is collapsible to its face $B^{n-1}$ and so on by induction. Moreover, a cone $c K$ on a compact polyhedron $K$ is collapsible (to its vertex). Indeed, note that for each simplex $A \subset K$, the cone $c A$ collapses from $A$ to $c(\partial A)$, hence $c K$ collapses to a point inductively by simplices of decreasing dimension.

A collapsing $K \searrow Y$ generates a deformation retraction $r: K \rightarrow Y$, given by deformations of each ball $B^{n}$ to its face $B^{n-1}$. Consider a homotopy $H_{t}$ between the identity map $K \rightarrow K$ and the deformation retraction $r: K \rightarrow Y$, given by the collapse $K \searrow Y$. The trace of a subpolyhedron $S$ of $K$ under the collapse $K \searrow Y$ is the union of $H_{t}(S)$ over $t \in[0,1]$ (this in fact depend on the homotopy not only on the collapse).

Suppose that $K$ is a subpolyhedron of a PL manifold $M$. A neighborhood $N$ of $K$ in $M$ is called regular, if $N$ is a compact bounded manifold and $N \searrow K$. The same polyhedron can have distinct regular neighborhoods. But the regular neighborhood is unique up to 
homeomorphism and even up to isotopy fixed on $K$ [RS72]. The regular neighborhood of a collapsible polyhedron is a ball [RS72]. (The inverse statement, i.e. that $K \subset B^{n}$ and $K \searrow *$ imply $B^{n} \searrow K$, is true only in codimension $\geq 3$ [Hu69].)

Let us fix the following convention. The notation $R_{M}(K)$ means 'a sufficiently small regular neighborhood of $K$ in $M$ ', when it first appears, and 'the regular neighborhood of $K$ in $M^{\prime}$, after the first appearance.

Proof of the Weber Theorem for the general case $2 m \geq 3 n+3$. The proof of the Weber Theorem 8.1 for the general case consists of two steps:

(1) the construction of a nondegenerate almost embedding (an analogue of Lemma 4.2, the details for this step are given as Proposition 8.4 below); and

(2) the construction of an embedding from a non-degenerate almost embedding (a generalization of Freedman-Krushkal-Teichner Lemma 4.3, the details are given in [Sk98, RS99]).

Proposition 8.4. Suppose that $N$ is an n-polyhedron with a triangulation $T, 2 m \geq 3 n+3$ and $\Phi: \widetilde{N} \rightarrow S^{m-1}$ is an equivariant map. Then for each $\sigma \times \tau \in \widetilde{T}$ such that $\sigma \leq \tau$ there exists a nondegenerate $P L$ map $f: N \rightarrow \mathbb{R}^{m}$ such that

(*) $f \alpha \cap f \beta=\emptyset$ for each $\alpha \times \beta<\sigma \times \tau$, and

(**) $\tilde{f} \simeq_{e q} \Phi$ on $J_{\sigma \tau}:=\cup\{\alpha \times \beta \cup \beta \times \alpha \subset \widetilde{T} \mid \alpha \times \beta<\sigma \times \tau\}$.

The Weber Theorem 8.1 follows from Proposition 8.4 by taking $\sigma$ and $\tau$ to be the last simplex of $T$ and then applying a generalization of the Freedman-Krushkal-Teichner Lemma 4.3 [Sk98]. The Weber Theorem 8.1 can also be proved by first constructing the immersion and then modifying it to an embedding [Sk02].

Proof of Proposition 8.4. Take a general position map $f: N \rightarrow \mathbb{R}^{m}$ that is linear on the simplices of the triangulation $T$. The map $f$ is already non-degenerate. By the induction hypothesis on $\sigma \times \tau$ we may assume that $f$ is non-degenerate and the properties $\left(^{*}\right)$ and $\left({ }^{* *}\right)$ hold. Suppose that $p+q \geq m-1$ (otherwise the inductive step holds by general position).

The first part of the proof (a generalization of Proposition 8.3 and the Whitney trick) is getting the property $f \sigma \cap f \tau=\emptyset$. The second part of the proof (a generalization of Proposition 8.2 and the van Kampen finger moves) is getting the property $\widetilde{f} \simeq_{e q} \Phi$ on $J_{\sigma \tau} \cup \sigma \times \tau \cup \tau \times \sigma$.

Constructions of balls $D_{\sigma}, D_{\tau}$ and $D^{m}$. The first step in the proof of Proposition 8.4 generalizes the construction of the $\operatorname{arcs} l_{1}, l_{2}$ and the disk $D$ in Whitney trick, or the construction of $D_{\sigma}, D_{\tau}$ and $D^{2 n}$ in the case $m=2 n$ above. Let $\Sigma=f \sigma \cap f \tau$. By $\left({ }^{*}\right)$ we have

$$
f \sigma \cap f \partial \tau=f \partial \sigma \cap f \tau=\emptyset .
$$

Hence $\Sigma=f \stackrel{\circ}{\circ} f \stackrel{\circ}{\tau}$. By general position, $\operatorname{dim} \Sigma \leq p+q-m$. Let $C_{\sigma} \subset \stackrel{\circ}{\sigma}$ be the trace of the polyhedron $\sigma \cap f^{-1} \tau$ under some collapse $\sigma \searrow$ (a point in $\stackrel{\circ}{\tau}$ ). Define analogously $C_{\tau} \subset \stackrel{\circ}{\tau}$. The polyhedra $C_{\sigma}, C_{\tau}$ are generalizations of the $\operatorname{arcs} l_{1}, l_{2}$ from the Whitney trick, and of $C_{\sigma}, C_{\tau}$ above. They are collapsible,

$$
\Sigma \subset f C_{\sigma} \cap f C_{\tau} \quad \text { and } \quad \operatorname{dim} C_{\sigma}, \operatorname{dim} C_{\tau} \leq p+q-m+1 .
$$


Consider a collapse from some PL $m$-ball $J^{m}$ in $\mathbb{R}^{m}$, containing $\Sigma$ in its interior, to a point in $J^{m}$. Let $C$ be the trail of $C_{\sigma} \cup C_{\tau}$ under this collapse. The polyhedron $C$ is a generalization of the disk $C$ from the Whitney trick. It is collapsible, it contains $C_{\sigma} \cup C_{\tau}$ and $\operatorname{dim} C \leq p+q-m+2$. Hence by general position, $C \cap f \sigma=C_{\sigma}$ and $C \cap f \tau=C_{\tau}$.

Take the regular neighborhoods of polyhedra $C_{\sigma}, C_{\tau}$ and $C$ in some sufficiently fine (agreeing) triangulations of $\sigma, \tau$ and $\mathbb{R}^{m}$, respectively. They are PL balls

$$
D_{\sigma}^{p} \subset \stackrel{\circ}{\sigma}, \quad D_{\tau}^{q} \subset \stackrel{\circ}{\tau} \text { and } D^{m} \subset \mathbb{R}^{m} \text { such that }
$$

(a) $\left.f\right|_{D_{\sigma}}$ and $\left.f\right|_{D_{\tau}}$ are proper embeddings into $D^{m}$;

(b) $f \sigma \cap f \tau \subset \stackrel{D}{D}^{m}$;

(c) $D_{\sigma}=\sigma \cap f^{-1} D^{m}$ and $D_{\tau}=\tau \cap f^{-1} D^{m}$;

(d) $D^{m} \cap f P=\emptyset$, where $P=N-$ st $\sigma-$ st $\tau$.

Only the last property needs a proof. By $\left(^{*}\right)$ we have $C_{\sigma} \cap P=\emptyset$. By general position,

$$
\operatorname{dim}(f P \cap f \tau) \leq n+q-m, \quad \text { hence } \quad \operatorname{dim}(f P \cap f \tau)+\operatorname{dim} C_{\tau}<q \quad \text { so } \quad C_{\tau} \cap P=\emptyset .
$$

Therefore $C \cap f P=\emptyset$, which implies (d).

A generalization of the Whitney trick. Take PL balls $D^{m}, D_{\sigma}$ and $D_{\tau}$ as above. Since $f$ is non-degenerate and $(*)$ holds, it follows that $f \partial D_{\sigma} \cap f D_{\tau}=f \partial D_{\tau} \cap f D_{\sigma}=\emptyset$. Since $m-p \geq 3$, it follows that a homotopy equivalence $h: D^{m}-f D_{\sigma} \rightarrow S^{m-p-1}$ is constructed analogously to the homotopy equivalence $S^{m}-f S^{q} \rightarrow S^{m-q-1}$ from the definition of the linking coefficients at the beginning of $\S 3$. The coefficient of the intersection of $\left.f\right|_{\partial D_{\sigma}}$ and $\left.f\right|_{\partial D_{\tau}}$ is the homotopy class

$$
I\left(\left.f\right|_{D_{\tau}},\left.f\right|_{D_{\tau}}\right):=\left[\left.f\right|_{\partial D_{\tau}}: \partial D_{\tau} \rightarrow D^{m}-f D_{\sigma} \stackrel{h}{\rightarrow} S^{m-p-1}\right] \in \pi_{q-1}\left(S^{m-p-1}\right) .
$$

We have

$$
\Sigma^{p} I\left(\left.f\right|_{D_{\sigma}},\left.f\right|_{D_{\tau}}\right)=(-1)^{m-p}\left[\left.\widetilde{f}\right|_{\partial\left(D_{\sigma} \times D_{\tau}\right)}\right]=\left[\left.\Phi\right|_{\partial\left(D_{\sigma} \times D_{\tau}\right)}\right]=0 .
$$

Here the first equality holds by [We67, Proposition 1]. The second equality holds since $\widetilde{f} \simeq \Phi$ on $\partial\left(D_{\sigma} \times D_{\tau}\right)$ by the inductive hypothesis. The third equality holds since $\Phi$ is defined over $\widetilde{T} \supset D_{\sigma} \times D_{\tau}$. Since

$$
2 p+q \leq 2 m-3, \quad \text { we have } q-1 \leq 2(m-p-1)-2 .
$$

So by the Freudenthal Suspension Theorem the homomorphism $\Sigma^{p}$ above is a monomorphism. Hence the embedding $\left.f\right|_{\partial D_{\tau}}$ extends to a map $f^{\prime}: D_{\tau} \rightarrow D^{m}-f D_{\sigma}$.

Since $2 q-m+1 \leq m-p-2$, by the Penroze-Whitehead-Zeeman-Irwin Embedding Theorem 2.6.c it follows that $f^{\prime}$ is homotopic rel $\partial D_{\tau}$ to an embedding $f^{+}: D_{\tau} \rightarrow$ $D^{m}-f D_{\sigma}$. Here we again use the inequality $p+2 q \leq 2 m-3$. Since $m-q \geq 3$, by the relative version of Theorem 2.5.a [Ze63, Corollary 1 to Theorem 9] it follows that there is an ambient isotopy $h_{t}: D^{m} \rightarrow D^{m}$ rel $\partial D^{m}$ carrying $\left.f\right|_{D_{\tau}}$ to $f^{+}$. Extend $f^{+}$over $N$ by the formula

$$
f^{+}(x)=\left\{\begin{array}{ll}
h_{1}(f(x)), & \text { if } f(x) \in D^{m} \\
f(x), & \text { otherwise }
\end{array} \text { and } x \in \gamma \quad \text { for some } \quad \gamma \supset \sigma^{q} .\right.
$$


It is easy to check that $f^{+}$is a non-degenerate PL map satisfying to the properties $\left(^{*}\right)$, $\left.{ }^{* *}\right)$ and such that $f^{+} \sigma \cap f^{+} \tau=\emptyset$.

A generalization of the van Kampen finger moves. We begin with the analogous construction of PL balls. By general position we can take points $C_{\sigma} \in \stackrel{\circ}{\sigma}$ and $C_{\tau} \in \stackrel{\circ}{\tau}$ such that the restrictions of $f$ to some small neighborhoods of $C_{\sigma}$ and $C_{\tau}$ are embeddings. Since $x, y \leq m-2$, we can join points $f C_{\sigma}$ and $f C_{\tau}$ by an arc $C \subset \mathbb{R}^{m}$ such that $C \cap f N=\left\{f C_{\sigma}, f C_{\tau}\right\}$. Let $D^{m}=R_{\mathbb{R}^{m}}(C)$. Then $f^{-1} D^{m}$ is the disjoint union of PL disks $D_{\sigma} \subset \stackrel{\circ}{\sigma}$ and $D_{\tau} \subset \stackrel{\circ}{\tau}$, which are regular neighborhoods in $N$ of $C_{\sigma}$ and $C_{\tau}$, respectively.

By the Borsuk Homotopy Extension Theorem, there is an extension $\Psi: \widetilde{T} \rightarrow S^{m-1}$ of the map $\left.\widetilde{f}\right|_{J_{\sigma \tau} \cup\left(\sigma \times \tau-\dot{D}_{\sigma} \times \stackrel{D}{\tau}_{\tau}\right)}$ such that $\Psi \simeq \Phi$. So $\Psi=\tilde{f}$ on $\partial\left(D_{\sigma} \times D_{\tau}\right)$. We may assume $\Psi=\Phi$. By [We67, Lemma 1], for each map

$$
f^{\prime}: D_{\sigma} \sqcup D_{\tau} \rightarrow D^{m} \quad \text { such that } \quad f^{\prime}=f \quad \text { on } \quad D_{\sigma} \sqcup \partial D_{\tau} \quad \text { and } \quad f^{\prime} D_{\sigma} \cap f^{\prime} D_{\tau}=\emptyset
$$

and homotopy $f_{t}$ rel $D_{\sigma} \sqcup \partial D_{\tau}$ from $f$ to $f^{\prime}$ we have

$$
\left[H_{\Phi \tilde{f}_{t} \tilde{f}^{\prime}}\right]=\left[H_{\Phi \tilde{f}}\right]+\left[H_{\tilde{f} \tilde{f}_{t} \tilde{f}^{\prime}}\right]=\left[H_{\Phi \tilde{f}}\right]+(-1)^{m-p} \Sigma^{p}\left[h_{f f^{\prime}}\right] \in \pi_{p+q}\left(S^{m-1}\right) .
$$

Here $\Phi, \widetilde{f}$ and $\widetilde{f}^{+}$denote the restrictions of these maps to $D_{\sigma} \times D_{\tau} ; \widetilde{f}_{t}$ denotes the restriction of this map to $\partial\left(D_{\sigma} \times D_{\tau}\right)$, and the maps $H$ are defined as in the second step of the proof of Proposition 8.2. Since

$$
2 p+q \leq 2 m-3, \quad \text { we have } q \leq 2(m-p-1)-1 .
$$

So by the Freudenthal Suspension Theorem $\Sigma^{p}$ is an epimorphism. Since for every element $\beta \in \pi_{q}\left(S^{m-p-1}\right)$ there is a map (not necessarily an embedding)

$$
f^{\prime}: D_{\tau} \rightarrow D^{m}-f D_{\sigma} \quad \text { such that } \quad\left[h_{f f^{\prime}}\right]=\beta \quad \text { and } \quad f^{\prime}=f \quad \text { on } \quad D_{\sigma} \sqcup \partial D_{\tau},
$$

it follows that we can take $f^{\prime}$ so that $\left[H_{\Phi \tilde{f}_{t} \tilde{f}^{\prime}}\right]=0$.

The rest of the proof is the same as in the generalization of the Whitney trick.

Generalization of the Weber Theorem. We illustrate some of the ideas of the proof of Theorem 5.5 by proving the following weaker result for $d \in\{0,1\}$.

Theorem 8.5. Suppose that $N$ is a d-connected closed $P L n$-manifold, $2 m \geq 3 n+2-d$, $m \geq n+3$ and $\Phi: \widetilde{N} \rightarrow S^{m-1}$ is an equivariant map. Then there is a PL embedding $f: N \rightarrow \mathbb{R}^{m}[S k 97]$.

Recall some classical results and their generalizations required to prove Theorem 8.5.

The Engulfing Lemma 8.6. Suppose that $N$ is a $(2 k+2-n)$-connected closed $n$ manifold and $K \subset N$ is a $k$-polyhedron such that $n-k \geq 3$ and the inclusion $K \subset N$ is null-homotopic. Then $K$ can be engulfed in $N$, i.e. is contained in an n-ball $B \subset N$ [PWZ61, Ze63]. 
Theorem 8.7. Suppose that $N$ is a closed homologically $(3 n-2 m+2)$-connected $P L$ $n$-manifold, $m-n \geq 3$ and $g: N \rightarrow \mathbb{R}^{m}$ is a map such that $\Sigma(g)$ is contained in some $P L$ $n$-ball $B \subset N$. Then there is an embedding $f: N \rightarrow \mathbb{R}^{m}$ such that $f=g$ on $N-\stackrel{\circ}{\text {. }}$.

Proof. The theorem is essentially proved in [Hi65, cf. Sk97, Theorem 2.1.2]. Let $M=$ $\mathbb{R}^{m}-\operatorname{Int} R(g(N-\stackrel{\circ}{B}), g \partial B)$. Since $N$ is homologically $(3 n-2 m+2)$-connected, we have by Alexander duality

$$
H_{i}(M) \cong H^{m-1-i}\left(\mathbb{R}^{m}-M\right) \cong H^{m-1-i}(N-\stackrel{\circ}{B}) \cong H_{n-m+1+i}(N)=0
$$

for $i \leq 2 n-m+1$. Since $m-n \geq 3$, it follows that $M$ is simply connected. Therefore by the Hurewicz Isomorphism Theorem we have that $M$ is $(2 n-m+1)$-connected. Hence by the Penrose-Whitehead-Zeeman-Irwin Embedding Theorem 2.6.c the embedding $g$ : $\partial B \rightarrow \partial M$ extends to an embedding $f: B \rightarrow M$. Extending $f$ by $g$ outside $B$ we complete the proof.

Theorem 8.8. Let $N$ be an $n$-polyhedron with triangulation $T$. If $m-n \geq 3$ and there exists an equivariant map $\Phi: \widetilde{N} \rightarrow S^{m-1}$, then there exists a general position nondegenerate $P L \operatorname{map} f: N \rightarrow \mathbb{R}^{m}$ such that

$$
f \sigma \cap f \tau=f(\sigma \cap \tau) \quad \text { if } \quad p=\operatorname{dim} \sigma \leq \operatorname{dim} \tau=q \quad \text { and } \quad p+q+n \leq 2 m-3 .
$$

Sketch of the proof. Analogous to the proof of the Weber Theorem 8.1. Recall that the Weber Theorem 8.1 is proved by induction on $\sigma \times \tau \in \widetilde{T}$. If $\operatorname{dim} \sigma=p$ and $\operatorname{dim} \tau=q$, then we need the following dimensional restrictions:

$p+2 q \leq 2 m-3$ to apply the Freudenthal Suspension Theorem (twice) and the PenroseWhitehead-Zeeman trick;

$$
p+q+n \leq 2 m-3 \text { to get the property } D^{m} \cap f(N-\text { st } \sigma-\text { st } \tau)=\emptyset .
$$

$A$ reduction of Theorem 8.5. It suffices to prove that

(***) For some fine triangulation $T$ of $N$ and a map $f: N \rightarrow \mathbb{R}^{m}$ as is given by Theorem 8.8, the inclusion $\Sigma(f) \subset N$ is homotopic to a map to some d-dimensional subpolyhedron of $N$.

Indeed, since $N$ is $d$-connected, (***) implies that the inclusion $\Sigma(f) \subset N$ is nullhomotopic. Since $N$ is $(2(2 n-m)-n+2)=d$-connected, by the Engulfing Lemma 8.6 it follows that $\Sigma(f)$ is contained in some PL $n$-ball in $N$. Then $N$ embeds into $\mathbb{R}^{m}$ by Theorem 8.7.

Note that if a map $f$ given by Theorem 8.8 is a PL immersion (i.e. a local embedding), then $\Sigma(f)$ does not intersect the $(2 m-2 n-3)$-skeleton of $T$. Hence it retracts to the $(n-1-(2 m-2 n-3))=d$-skeleton of a triangulation, dual to $T$, i.e. (***) holds.

Proof of $\left({ }^{* *}\right)$ for $d=0$. We may assume that $2 m=3 n+2$. Hence

$$
f \sigma \cap f \tau=f(\sigma \cap \tau) \quad \text { unless } \quad \operatorname{dim} \sigma=\operatorname{dim} \tau=n .
$$

For $n$-simplices $\alpha$ and $\beta$ let

$$
S_{\alpha \beta}=\left\{\begin{array}{ll}
\alpha \cap f^{-1} f \beta & \alpha \cap \beta=\emptyset \\
f^{-1} \operatorname{Cl}[(f \alpha \cap f \beta)-f(\alpha \cap \beta)] & \alpha \cap \beta \neq \emptyset
\end{array} .\right.
$$




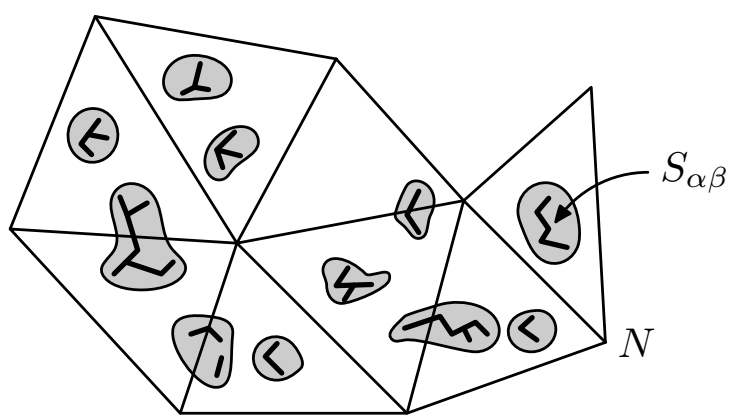

Figure 8.7

$$
\text { Then } \quad \Sigma(f)=\bigcup_{\alpha \neq \beta} S_{\alpha \beta} \quad \text { and } \quad S_{\alpha \beta} \cap S_{\gamma \delta}=\emptyset \quad \text { when } \quad \alpha \beta \neq \gamma \delta \text {. }
$$

Here $\alpha \beta$ is the ordered pair $(\alpha, \beta)$ when $\alpha \cap \beta=\emptyset$ and the non-ordered pair $\{\alpha, \beta\}$ when $\alpha \cap \beta \neq \emptyset$. Therefore the contractibility of $\alpha$ (and of $\alpha \cup \beta$ for $\alpha \cap \beta \neq \emptyset$ ) implies (***).

Proof of (***) for $d=1$. We may assume that $2 m=3 n+1$. Define $\alpha \beta$ and $S_{\alpha \beta}$ as in the case $d=0$. We denote such pairs $\alpha \beta$ by Latin letters $i, j, k, l$. First we prove that

(a) $S_{i} \cap S_{j} \cap S_{k}=\emptyset$ for distinct $i, j, k=1, \ldots, s$;

(b) For each $i=1, \ldots, s$ there is a contractible polyhedron $A_{i} \subset N$, containing $S_{i}$.

If $S_{i} \cap S_{j} \neq \emptyset$, then there is a contractible polyhedron $A_{i j} \subset N$, containing $A_{i} \cup A_{j}$.

Indeed, we may assume that a triangulation $T$ of $N$ is such that, for each $x \in N$, the star $\operatorname{st}^{2} x=$ st st $x$ is contractible. By Theorem $8.8 S_{\alpha \beta} \neq \emptyset$ is possible only when

$$
\text { either } \quad \operatorname{dim} \alpha=\operatorname{dim} \beta=n \quad \text { or } \quad\{\operatorname{dim} \alpha, \operatorname{dim} \beta\}=\{n, n-1\} \text {. }
$$

By general position, $f$ has no triple points. Therefore each non-empty intersection of any three of $S_{1}, \ldots, S_{s}$ can be only of the form

$$
\begin{gathered}
S_{\alpha_{1} \beta} \cap S_{\alpha_{2} \beta} \cap S_{\alpha_{3} \beta}=S_{\alpha \beta} \quad\left(\text { or } S_{\beta \alpha_{1}} \cap S_{\alpha_{2} \beta} \cap S_{\beta \alpha_{3}}=S_{\beta \alpha}\right) \\
\text { for some } \quad \alpha_{1}^{n}, \alpha_{2}^{n}, \alpha_{3}^{n}, \beta^{n}, \alpha \in T, \quad \alpha=\alpha_{1} \cap \alpha_{2} \cap \alpha_{3} .
\end{gathered}
$$

Since $S_{\alpha \beta} \neq \emptyset$, it follows that $\operatorname{dim} \alpha=n-1$. Since $N$ is a closed manifold, then no three distinct $n$-simplices of $T$ intersect by an $(n-1)$-simplex of $T$. This contradiction shows that (a) is true.

Let

$$
A_{\alpha \beta}= \begin{cases}\alpha & \alpha \cap \beta=\emptyset, \\ \alpha \cup \beta & \alpha \cap \beta \neq \emptyset .\end{cases}
$$

If $S_{i} \cap S_{j} \neq \emptyset$, then take a point $a_{i j} \in S_{i} \cap S_{j}$ and let $A_{i j}=$ st $^{2} a_{i j}$. From the definition of $S_{i}$ and $A_{i}$ it follows that $S \subset A_{i}$ and $A_{i} \cup A_{j} \subset A_{i j}$. By the choice of $T, A_{i}$ and $A_{i j}$ are contractible.

Now we construct a homotopy of $\Sigma(f)$ onto its 'reduced' nerve. From (a) it follows that the sets $S_{i} \cap S_{j}$ are disjoint for distinct non-ordered pairs $i, j=1, \ldots, s$. Take disjoint regular neighborhoods $U_{i j}$ of $S_{i} \cap S_{j}$ in $\bigcup_{i=1}^{s} S_{i}$. Since $A_{i}$ is contractible, it follows that 
there is a homotopy $F_{i}: \mathrm{Cl}\left(S_{i}-\bigcup_{j \neq i} U_{i j}\right) \times I \rightarrow A_{i}$ between the inclusion and a constant map to some point $a_{i} \in A_{i}$.

Suppose that $S_{i} \cap S_{j} \neq \emptyset$. Since $A_{i j}$ is contractible, it follows that there is an arc $l_{i j} \subset A_{i j}$ joining $a_{i}$ and $a_{j}$. Also we can extend homotopies $F_{i}$ and $F_{j}$ over $U_{i j}$ to a homotopy $F_{i j}: U_{i j} \times I \rightarrow N$ between the inclusion and a map of $U_{i j}$ to $l_{i j}$ (we can do this first for vertices and then for edges). Since all $U_{i j}$ are disjoint and all $F_{i j}$ are extensions of $F_{i}$ and $F_{j}$, then all the constructed homotopies define a homotopy $F:\left(\bigcup_{i=1}^{s} S_{i}\right) \times I \rightarrow N$ between the inclusion and a map onto the following subgraph of $N$ :

$$
\left(\bigcup_{i=1}^{s} a_{i}\right) \cup\left(\bigcup\left\{l_{i j} \mid 1 \leq i<j \leq s \text { and } S_{i} \cap S_{j} \neq \emptyset\right\}\right) \text {. }
$$

From our proof it follows that Theorem 8.5 for $2 m \geq 3 n+1$ is true even if there only exists an equivariant map to $S^{m-1}$ from the $\left(\left[\frac{4 m}{3}\right]-2\right)$-skeleton of $\widetilde{T}$.

It would be interesting to know if Theorem 8.5 remains true when $N$ has singularities of dimension at most $m-n-2$. This is not clear, contrary to what is written in [Sk97] (because e.g. in the suspension of a homology sphere the complement to vertices is not simply-connected so we cannot apply Engulfing Lemma 8.6).

\section{REFERENCES}

[Ad93] M. Adachi, Embeddings and Immersions, Transl. of Math. Monographs 124, Amer. Math. Soc., 1993.

[Ak69] E. Akin, Manifold phenomena in the theory of polyhedra, Trans. Amer. Math. Soc. 143 (1969), 413-473.

[Ak96] P. M. Akhmetiev, On isotopic and discrete realization of mappings from $n$-dimensional sphere to Euclidean space (in Russian), Mat. Sbornik 187:7 (1996), 3-34; English transl., Sb. Math. 187:7 (1996), 951-980.

[Ak96'] P. M. Akhmetiev, Mapping an n-sphere in a 2n-Euclidean space: its realization (in Russian), Trudy Mat. Inst. im. Steklova 212 (1996), 37-45; English transl. in Proc. Math. Steklov Inst., 212 (1996), 32-39.

[Ak96"] P. M. Akhmetiev, Prem-mappings, triple points of orientable surface and Rohlin Signature Theorem (in Russian), Mat. Zametki 59:6 (1996), 803-810; English transl. in Math. Notes 59:6 (1996), 581-585.

[Ak00] P. M. Akhmetiev, Embeddings of compacta, stable homotopy groups of spheres and singularity theory (in Russian), Uspekhi Mat. Nauk 55:3 (2000), 3-62; English transl. in Russ. Math. Surveys

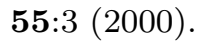

[Al24] J. W. Alexander, On the subdivision of 3-space by polyhedron, Proc. Nat. Acad. Sci. USA 10 (1924), 6-8.

[ARS01]P. Akhmetiev, D. Repovš and A. Skopenkov, Embedding products of low-dimensional manifolds in $\mathbb{R}^{m}$, Topol. Appl. 113 (2001), 7-12.

[ARS02]P. Akhmetiev, D. Repovš and A. Skopenkov, Obstructions to approximating maps of n-surfaces to $\mathbb{R}^{2 n}$ by embeddings, Topol. Appl. 123 (2002), 3-14.

[Ba75] D. R. Bausum, Embeddings and immersions of manifolds in Euclidean space, Trans. AMS 213 (1975), 263-303.

[BH70] J. Boechat and A. Haefliger, Plongements differentiables de varietes de dimension 4 dans $\mathbb{R}^{7}$, Essays on topology and related topics (Springer,1970) (1970), 156-166.

[BKK02]M. Bestvina, M. Kapovich and B. Kleiner, Van Kampen's embedding obstruction for discrete groups, Invent. Math. 150:2 (2002), 219-235. 
[BM99] R. L. Bryant and W. Mio, Embeddings of homology manifolds in codimension $\geq 3$, Topology 38:4 (1999), 811-821.

[BM00] R. L. Bryant and W. Mio, Embeddings in generalized manifolds, Trans. Amer. Math. Soc. 352:3 (2000), 1131-1137.

[Bo33] K. Borsuk, Über stetige Abbildungen der euklidischen Räume, Fund. Math. 21 (1933), 236-246.

[Bo71] J. Boechat, Plongements differentiables de varietes de dimension $4 k$ dans $\mathbb{R}^{6 k+1}$, Comment. Math. Helv. 46:2 (1971), 141-161.

[Br68] W. Browder, Embedding smooth manifolds, Proc. Int. Congr. Math. Moscow 1966 (1968), 712719 .

[Br71] R. L. Brown, Immersions and embeddings up to cobordism, Canad. J. Math 23 (1971), 1102-1115.

[Br72] J. L. Bryant, Approximating embeddings of polyhedra in codimension 3, Trans. Amer. Math. Soc. 170 (1972), 85-95.

[Ca86] A. Casson, Three lectures on new infinite constructions in 4-dimensional manifolds, A la Recherche de la Topologie Perdue, Progress in Mathematics, 62 (L. Guillou, A. Marin, eds.), Birkhauser, Boston, 1986, pp. 201-244.

[Ch69] A. V. Chernavskii, Piecewise linear approximations of embeddings of cells and spheres in codimensions higher than two, Mat. Sb. 80 (1969), no. (122), 339-364, Math. USSR Sb. 9 (1969), $321-344$.

[Cl34] S. Claytor, Topological immersions of peanian continua in a spherical surface, Ann. of Math. 35 (1934), 809-835.

[Cl37] S. Claytor, Peanian continua not embeddable in a spherical surface, Ann. of Math. 38 (1937), 631-646.

[CF60] P. E. Conner and E. E. Floyd, Fixed points free involutions and equivariant maps, Bull. AMS 66 (1960), 416-441.

[CG83] J. H. Conway and C. M. A. Gordon, Knots and links in spatial graphs, J. Graph Theory 7 (1983), 445-453.

[Co69] G. Cooke, Embedding certain complexes up to homotopy in Euclidean space, Ann. of Math. 90 (1969), 144-156.

[Co85] R. L. Cohen, The immersion conjecture for differentiable manifolds, Ann. of Math. 122 (1985), $237-328$.

[CRS98] A. Cavicchioli, D. Repovš and A. B. Skopenkov, Open problems on graphs, arising from geometric topology, Topol. Appl. 84 (1998), 207-226.

[CRS04]M. Cencelj, D. Repovš and A. Skopenkov, On the Browder-Levine-Novikov embedding theorems, Trudy MIRAN 247 (2004), 280-290; Proc. Steklov Math. Inst. 247 (2004).

[CRS05]M. Cencelj, D. Repovš and M. Skopenkov, Classification of framed links in 3-manifolds, submitted.

[CRS] M. Cencelj, D. Repovš and M. Skopenkov, Knotted tori and the $\beta$-invariant, preprint.

[CS79] S. E. Cappell and J. L. Shaneson, Imbeddings and immersions of 4-dimensional manifolds in $\mathbb{R}^{6}$, Geometric topology (J. C. Cantrell, eds.), Academic press, New York, 1979, pp. 301-303.

[CW78] F. X. Conolly and B. Williams, Embedding up to homotopy and geometric suspension of manifolds, Quart. J. Math. Oxford (2) 29 (1978), 385-401.

[Da86] R. J. Daverman, Decompositions of manifolds, Academic Press, New York, 1986.

[Do87] S. K. Donaldson, The orientation of Yang-Mills moduli spaces and 4-manifold topology, J. Diff. Geom. 26 (1987), 397-428.

[DP97] T. J. Dodson and P. E. Parker, A User's Guide to Algebraic Topology, Kluwer, Doldrecht-BostonLondon, 1997.

[DRS91]A. N. Dranišnikov, D. Repovš and E. V. Ščepin, On intersection of compacta of complementary dimension in Euclidean space, Topol. Appl 38 (1991), 237-253.

[DRS93]A. N. Dranišnikov, D. Repovš and E. V. Ščepin, On intersection of compacta in Euclidean space: the metastable case, Tsukuba J.Math. 17 (1993), 549-564.

[Ed75] R. B. Edwards, The equivalence of close piecewise linear embeddings, General Topology and its Applications 5 (1975), 147-180.

[FF89] A. T. Fomenko and D. B. Fuchs, A course in homotopy theory, Nauka, Moscow, 1989. (in Russian)

[FKT94]M. H. Freedman, V. S. Krushkal and P. Teichner, Van Kampen's embedding obstruction is incomplete for 2-complexes in $\mathbb{R}^{4}$, Math. Res. Letters 1 (1994), 167-176. 
[FKV87]S. M. Finashin, M. Kreck and O. Ya. Viro, Exotic knottings of surfaces in the 4-sphere, Bull. Amer. Math. Soc. 17:2 (1987), 287-290.

[FKV88]S. M. Finashin, M. Kreck and O. Ya. Viro, Non-diffeomorphic but homeomorphic knottings of surfaces in the 4-sphere, Lecture Notes in Math. 1346 (1988), 157-198.

[Fl34] A. Flores, Über n-dimensionale Komplexe die im $E^{2 n+1}$ absolute Selbstverschlungen sind, Ergeb. Math. Koll. 6 (1934), 4-7.

[FQ90] M. H. Freedman and F. S. Quinn, Topology of 4-Manifolds, Princeton Univ. Press, Princeton, 1990.

[Fr87] G. K. Francis, A Topological Picturebook, Springer-Verlag, Berlin, 1987.

[FS59] D. B. Fuchs and A. S. Schwarz, Cyclic powers of polyhedra and the imbedding problem, Dokl. Akad. Nauk SSSR 125 (1959), 285-288. (in Russian)

[Fu94] F. Fuquan, Embedding four manifolds in $\mathbb{R}^{7}$, Topology 33:3 (1994), 447-454.

[Fu02] F. Fuquan, Orientable 4-manifolds topologically embed into $\mathbb{R}^{7}$, Topology 41 (2002), 927-930.

[Ga92] M. Galecki, On embeddability of $C W$-complexes in Euclidean space, preprint, Univ. of Tennessee, Knoxville, 1992.

[Gi71] S. Gitler, Embedding and immersion of manifolds, Proc. Symp. Pura Appl. Math., AMS, Providence 22 (1971), 87-96.

[Gl63] H. Gluck, Unknotting $S^{1}$ in $S^{4}$, Bull. Amer. Math. Soc. 69:1 (1963), 91-94.

[Gl68] H. Gluck, Geometric characterization of differentiable manifolds in Euclidean space, II, Michigan Math. J. 15:1 (1968), 33-50.

[GMR94D. Gillman, S. V. Matveev and D. Rolfsen, Collapsing and reconstruction of manifolds, Contemp. Math. 164 (1994), 35-39.

[Go72] C. M. A. Gordon, Embeddings of PL-manifolds with boundary, Proc. Camb. Phil. Soc. 72 (1972), 21-25.

[Gr86] M. Gromov, Partial differential relations, Ergebnisse der Mathematik und ihrer Grenzgebiete (3), Springer Verlag, Berlin-New York, 1986.

[GR92] D. Gillman and D. Rolfsen, 3-manifolds embed in small 3-complexes, Intern. J. Math. 3 (1992), $179-183$.

[GS06] D. Goncalves and A. Skopenkov, Embeddings of homology equivalent manifolds with boundary, Topol. Appl. (2006), to appear.

[Gu53] V. K. A. M. Gugenheim, Piecewise linear isotopy and embedding of elements and spheres, I, Proc. of the London Math. Soc. 3 (1953), 29-53.

[GW99] T. Goodwillie and M. Weiss, Embeddings from the point of view of immersion theory, II, Geometry and Topology 3 (1999), 103-118.

[Ha61] A. Haefliger, Plongements differentiables de varietes dans varietes, Comment. Math. Helv. 36 (1961), 47-82.

[Ha62] A. Haefliger, Knotted $(4 k-1)$-spheres in 6k-space, Ann. of Math. 75 (1962), 452-466.

[Ha62'] A. Haefliger, Differentiable links, Topology 1 (1962), 241-244.

[Ha62"] A. Haefliger, Plongements de varietes dans le domain stable, Seminare Bourbaki 245 (1962).

[Ha63] A. Haefliger, Plongements differentiables dans le domain stable, Comment. Math. Helv. 36 (196263), 155-176.

[Ha66] A. Haefliger, Differentiable embeddings of $S^{n}$ in $S^{n+q}$ for $q>2$, Ann. Math., Ser.3 83 (1966), $402-436$.

[Ha66'] A. Haefliger, Enlacements de spheres en codimension superiure a 2, Comment. Math. Helv. 41 (1966-67), 51-72.

[Ha67] A. Haefliger, Lissage des immersions-I, Topology 6 (1967), 221-240.

[Ha68] A. Haefliger, Knotted spheres and related geometric topic, In: Proc. of the Int. Cong. Math., Moscow, Mir (1968), 437-445.

[Ha68'] D. D. J. Hacon, Embeddings of $S^{p}$ in $S^{1} \times S^{q}$ in the metastable range, Topology 7 (1968), 1-10.

[Ha69] L. S. Harris, Intersections and embeddings of polyhedra, Topology 8 (1969), 1-26.

[Ha] A. Haefliger, Lissage des immersions-II, preprint (1966).

[Ha84] N. Habegger, Obstruction to embedding disks II: a proof of a conjecture by Hudson, Topol. Appl. 17 (1984), 123-130.

[Ha86] N. Habegger, Classification of links in $S^{1} \times \mathbb{R}^{n-1}$, Topology 25:3 (1986), 253-260. 
[HH62] A. Haefliger and M. W. Hirsch, Immersions in the stable range, Ann. of Math. 75:2 (1962), $231-241$.

[HH63] A. Haefliger and M. W. Hirsch, On existence and classification of differential embeddings, Topology 27 (1963), 129-135.

[HK98] N. Habegger and U. Kaiser, Link homotopy in 2-metastable range, Topology 37:1 (1998), 75-94.

[Hi61] M. W. Hirsch, The embedding of bounding manifold in euclidean space, Ann. of Math. 74 (1961), 494-497.

[Hi65] M. W. Hirsch, On embedding 4-manifolds in $\mathbb{R}^{7}$, Proc. Camb. Phil. Soc. 61 (1965).

[Hi66] M. W. Hirsch, Embeddings and compressions of polyhedra and smooth manifolds, Topology 4:4 (1966), 361-369.

[HL71] J. F. P. Hudson and W. B. R. Lickorish, Extending piecewise linear concordances, Quart. J. Math. (2) 22 (1971), 1-12.

[HLS65] W. C. Hsiang, J. Levine and R. H. Sczarba, On the normal bundle of a homotopy sphere embedded in Euclidean space, Topology 3 (1965), 173-181.

[Ho69] K. Horvatič, Embedding manifolds with low-dimensional spine, Glasnik Mat. 4(24):1 (1969), 101-116.

[Ho71] K. Horvatič, On embedding polyhedra and manifolds, Trans. AMS 157 (1971), 417-436.

[HS64] W. C. Hsiang and R. H. Szarba, On the embeddability and non-embeddability of sphere bundles over spheres, Ann. of Math. 80:2 (1964), 397-402.

[Hu59] S. T. Hu, Homotopy Theory, Academic Press, New York, 1959.

[Hu60] S. T. Hu, Isotopy invariants of topological spaces, Proc. Royal Soc. A255 (1960), 331-366.

[Hu63] J. F. P. Hudson, Knotted tori, Topology 2 (1963), 11-22.

[Hu63'] J. F. P. Hudson, Non-embedding theorem, Topology 2 (1963), 123-128.

[Hu66] J. F. P. Hudson, Extending piecewise linear isotopies, Proc. London Math. Soc. (3) 16 (1966), 651-668.

[Hu67] J. F. P. Hudson, PL embeddings, Ann. of Math. 85:1 (1967), 1-31.

[Hu69] J. F. P. Hudson, Piecewise-Linear Topology, Benjamin, New York, Amsterdam, 1969.

[Hu70] J. F. P. Hudson, Concordance, isotopy and diffeotopy, Ann. of Math. 91:3 (1970), 425-448.

[Hu70'] J. F. P. Hudson, Obstruction to embedding disks, In: Topology of manifolds (1970), 407-415.

[Hu72] J. F. P. Hudson, Embeddings of bounded manifolds, Proc. Camb. Phil. Soc. 72 (1972), 11-20.

[HZ64] J. F. P. Hudson and E. C. Zeeman, On regular neighborhoods, Correction to 'On regular neighborhoods', Proc. Lond. Math. Soc. (3), 21 (1970) 513-524., Proc. Lond. Math. Soc. (3) 14 (1964), 719-745.

[Ir65] M. C. Irwin, Embeddings of polyhedral manifolds, Ann. of Math. (2) 82 (1965), 1-14.

[Ka32] E. R. van Kampen, Komplexe in euklidische Raumen, Abb. Math. Sem. Hamburg 9 (1932), 72-78; berichtigung dazu, 152-153.

[Ke59] M. Kervaire, An interpretation of $G$. Whitehead's generalization of H. Hopf's invariant, Ann. of Math. 62 (1959), 345-362.

[Ke69] M. A. Kervaire, Smooth homology spheres and their fundamental groups, Trans. Amer. Math. Soc. 144 (1969), 67-72.

[Ke79] C. Kearton, Obstructions to embeddings and isotopy in the metastable range, Math. Ann. 243 (1979), 103-113.

[Ki84] R. Kirby, 4-manifold problems, Contemp. Math. 35 (1984), 513-528.

[Ki89] R. C. Kirby, The Topology of 4-Manifolds, Lect. Notes Math. 1374 (1989), Springer-Verlag, Berlin.

[KM61] A. Kervaire and J. W. Milnor, On 2-spheres in 4-manifolds, Proc. Nat. Acad. Sci. USA 47 (1961), 1651-1657.

[Ko62] A. Kosinski, On Alexander's theorem and knotted tori, In: Topology of 3-Manifolds, PrenticeHall, Englewood Cliffs, Ed. M. K. Fort, N.J. (1962), 55-57.

[Ko88] U. Koschorke, Link maps and the geometry of their invariants, Manuscripta Math. 61:4 (1988), $383-415$.

[Ko90] U. Koschorke, On link maps and their homotopy classification, Math. Ann. 286:4 (1990), 753782.

[Kr00] V. S. Krushkal, Embedding obstructions and 4-dimensional thickenings of 2-complexes, Proc. Amer. Math. Soc. 128:12 (2000), 3683-3691. 
[KS05] M. Kreck and A. Skopenkov, Inertia groups of smooth embeddings, submitted; http:// arxiv.org/ abs/ math.GT/ 0512594.

[Ku30] K. Kuratowski, Sur les problèmes des courbes gauche en topologie, Fund. Math. 15 (1930), 271283.

[Ku00] V. Kurlin, Basic embeddings into products of graphs, Topol. Appl. 102 (2000), 113-137.

[KW85] J. Keesling and D. C. Wilson, Embedding $T^{n}$-like continua in Euclidean space, Topol. Appl. 21 (1985), 241-249.

[La82] J. Lannes, La conjecture des immersions, Astérisque 92/93 (1982), 331-346.

[La96] M. Lackenby, The Whitney Trick, Topol. Appl. 71 (1996), 115-118.

[Le62] J. Levine, Notices of the Amer. Math. Soc. (1962).

[Le65] J. Levine, A classification of differentiable knots, Ann. of Math. 82 (1965), 15-50.

[Li65] W. B. R. Lickorish, The piecewise linear unknotting of cones, Topology 4 (1965), 67-91.

[Li75] A. Liulevicius, Immersions up to cobordism, Illinois J. Math 19 (1975), 149-164.

[LS69] W. B. R. Lickorish and L. C. Siebenmann, Regular neighborhoods and the stable range, Trans. AMS 139 (1969), 207-230.

[MA41] S. McLane and V. W. Adkisson, Extensions of homeomorphisms on the spheres, Michig. Lect. Topol. (1941), Ann Arbor, 223-230.

[Ma60] W. S. Massey, On the Stiefel-Whitney classes of a manifold, 1, Amer. J. Math 82 (1960), 92-102.

[Ma62] W. S. Massey, On the Stiefel-Whitney classes of a manifold, 2, Proc. AMS 13 (1962), 938-942.

[Ma90] W. S. Massey, Homotopy classification of 3-component links of codimension greater than 2, Topol. Appl. 34 (1990), 269-300.

[Ma80] R. Mandelbaum, Four-Dimensional Topology: An introduction, Bull. Amer. Math. Soc. (N.S.) 2 (1980), 1-159.

[Mc67] M. C. McCord, Embedding P-like compacta in manifolds, Canad. J. Math. 19 (1967), 321-332.

[Me02] S. Melikhov, On maps with unstable singularities, Topol. Appl. 120 (2002), 105-156.

[Me04] S. Melikhov, Sphere eversions and realization of mappings; http:// front.math.ucdavis.edu/ math.GT/ 0305158, Trudy MIRAN 247 (2004), 1-21, 159-181.

[Mi54] J. Milnor, Link groups, Ann. of Math. 59 (1954), 177-195.

[Mi65] J. Minkus, On embeddings of highly connected manifolds, Trans. of the Amer. Math. Soc. 115 (1965), 525-540.

[Mi70] R. T. Miller, Close isotopies on piecewise-linear manifolds, Trans. of the Amer. Math. Soc. 151:2 (1970), 597-628.

[Mi72] R. T. Miller, Approximating codimension 3 embeddings, Ann. of Math 95 (1972), 406-416.

[Mi72'] R. J. Milgram, On the Haefliger's groups, Bull. of the Amer. Math. Soc. 78:5 (1972), 861-865.

[Mi75] K. C. Millett, Piecewise linear embeddings of manifolds, Illinois J. Math. 19 (1975), 354-369.

[Mi97] P. Minc, Embedding simplicial arcs into the plane, Topol. Proc. 22 (1997), 305-340.

[MK58] J. Milnor and M. Kervaire, Bernoulli numbers, homotopy groups of spheres and a theorem of Rohlin, Proc. Int. Cong. Math., Edinburgh (1958).

[MR71] R. J. Milgram and E. Rees, On the normal bundle to an embedding, Topology 10 (1971), 299-308.

[MR86] W. Massey and D. Rolfsen, Homotopy classification of higher dimensional links, Indiana Univ. Math. J. 34 (1986), 375-391.

[MRS03]J. Malešič, D. Repovš and A. Skopenkov, On incompleteness of the deleted product obstruction for embeddings, Bol. Soc. Mat. Mexicana (3) 9 (2003), 165-170.

[MS67] S. Mardešić and J. Segal, e-mappings and generalized manifolds, Michigan Math. J. 14 (1967), $171-182$.

[MS74] J. W. Milnor and J. D. Stasheff, Characteristic Classes, Ann. of Math. St. 76, Princeton Univ. Press, Princeton, NJ, 1974.

[MS04] J. Mukai and A. Skopenkov, A direct summand in a homotopy group of the mod 2 Moore space, Kyushu J. Math. 58:1 (2004), 203-209.

[MT95] M. Mahowald and R. D. Thompson, The EHP Sequence and Periodic Homotopy, in: Handbook of Algebraic Topology, ed. I. M. James (1995), Elsevier Science B. V..

[Ne68] L. Neuwirth, An algorithm for the construction of 3-manifolds from 2-complexes, Proc. Camb. Phil. Soc. 64 (1968), 603-613.

[Ne98] S. Negami, Ramsay-type theorem for spatial graphs, Jour. Comb. Th. Ser B 72 (1998), 53-62. 
[No61] S. P. Novikov, Imbedding of simply-connected manifolds in Euclidean space, Dokl. Akad. Nauk SSSR 138 (1961), 775-778. (in Russian)

[Oo86] H. Ooshima, Whitehead products in the Stiefel manifolds and Samelson products in classical groups, Adv. Stud. in Pure Math. 9 (1986), 237-258.

[Pa56] G. F. Paechter, On the groups $\pi_{r}\left(V_{m n}\right)$, Quart. J. Math. Oxford, Ser.2; I 7:28 (1956), 249-265; II 9:33 (1958), 8-27; III 10:37 (1959), 17-37; IV 10:40 (1959), 241-260; V 11:41 (1960), 1-16.

[Po42] L. S. Pontryagin, Characteristic cycles of smooth manifolds, Dokl. Akad. Nauk SSSR 35:2 (1942), 35-39. (in Russian)

[Po85] M. M. Postnikov, Homotopy theory of $C W$-complexes, Nauka, Moscow, 1985. (in Russian)

[Pr66] T. M. Price, Equivalence of embeddings of $k$-complexes in $E^{n}$ for $n \geq 2 k+1$, Michigan Math. J. 13 (1966), 65-69.

[Pr04] V. V. Prasolov, Introduction into combinatorial and differential topology, AMS, Providence, RI, 2004.

[Pr] V. V. Prasolov, Elements of homology theory (in Russian), 2005.

[PS05] V. Prasolov and M. Skopenkov, Ramsay link theory (in Russian), Mat. Prosveschenie 9 (2005).

[PWZ61R. Penrose, J. H. C. Whitehead and E. C. Zeeman, Embeddings of manifolds in a Euclidean space, Ann. of Math. (2) 73 (1961), 613-623.

[RBS99]D. Repovš, N. Brodsky and A. B. Skopenkov, A classification of 3-thickenings of 2-polyhedra, Topol. Appl. 94 (1999), 307-314.

[Re71] E. Rees, Some embeddings of Lie groups in Euclidean spaces, Mathematika 18 (1971), 152-156.

[Re90] E. Rees, Problems concerning embeddings of manifolds, Advances in Math. 19:1 (1990), 72-79.

[Ro65] V. A. Rohlin, The embedding of non-orientable three-dimensional manifolds in the five-dimensional Euclidean space, Dokl. Akad. Nauk SSSR 160 (1965), 549-551. (in Russian)

[RS72] C. P. Rourke and B. J. Sanderson, Introduction to Piecewise-Linear Topology, Ergebn. der Math. 69, Springer-Verlag, Berlin, 1972.

[RS96] D. Repovš and A. B. Skopenkov, Embeddability and isotopy of polyhedra in Euclidean spaces, Trudy Math. Inst. Ross. Akad. Nauk 212 (1996); Proc. of the Steklov Inst. Math. 212 (1996), $173-188$.

[RS98] D. Repovš and A. B. Skopenkov, A deleted product criterion for approximability of a map by embeddings, Topol. Appl. 87 (1998), 1-19.

[RS99] D. Repovs and A. Skopenkov, New results on embeddings of polyhedra and manifolds into Euclidean spaces, Uspekhi Mat. Nauk 54:6 (1999), 61-109 (in Russian); English transl., Russ. Math. Surv., 1149-1196.

[RS99'] D. Repovš and A. B. Skopenkov, Borromean rings and embedding obstructions (in Russian), Trudy Math. Inst. Ross. Akad. Nauk 225 (1999), 331-338; English transl.:, Proc. of the Steklov Inst. Math. 225 (1999), 314-321.

[RS00] D. Repovš and A. Skopenkov, Obstruction theory for beginners (in Russian), Mat. Prosveschenie 4 (2000).

[RS01] C. Rourke and B. Sanderson, The compression theorem. I, II, Geom. Topol. (electronic) 5 (2001), 399-429, 431-440.

[RS01'] D. Repovš and A. Skopenkov, On contractible n-dimensional compacta, non-embeddable into $\mathbb{R}^{2 n}$, Proc. Amer. Math. Soc. 129 (2001), 627-628.

[RS02] D. Repovš and A. Skopenkov, On projected embeddings and desuspension of the $\alpha$-invariant, Topol. Appl. 124 (2002), 69-75.

[RS02'] D. Repovš and A. Skopenkov, Characteristic classes for beginners (in Russian), Mat. Prosveschenie 6 (2002), 60-77.

[RSS95] D. Repovš, A. B. Skopenkov and E. V. Ščepin, On embeddability of X $\times I$ into Euclidean space, Houston J. Math. 21 (1995), 199-204.

[RSS95']D. Repovš, A. B. Skopenkov and E. V. Ščepin, On uncountable collections of continua and their span, Colloq. Math. 69:2 (1995), 289-296.

[RST95]N. Robertson, P. D. Seymor and R. Thomas, Sachs' linkless embedding conjecture, J. Combin. Theory, Ser. B 64:2 (1995), 185-227.

[Ru73] T. B. Rushing, Topological Embeddings, Academic Press, New York, 1973.

[Ru82] D. Ruberman, Imbeddings four-manifolds and slicing links, Math. Proc. Camb. Phil. Soc 91 (1982), 107-110. 
[Sa65] R. De Sapio, Embedding $\pi$-manifolds, Ann. of Math. (2) 82 (1965), 213-224.

[Sa81] H. Sachs, On spatial representation of finite graphs, in: Finite and infinite sets, Colloq. Math. Soc. Janos Bolyai, North Holland, Amsterdam 37 (1981).

[Sa91] K. S. Sarkaria, Kuratowski complexes, Topology 30 (1991), 67-76.

[Sa91'] K. S. Sarkaria, A one-dimensional Whitney trick and Kuratowski's graph planarity criterion, Israel J. Math. 73 (1991), 79-89.

[Sc77] M. Scharlemann, Isotopy and cobordism of homology spheres in spheres, J. London Math. Soc., Ser. 2 16:3 (1977), 559-567.

[Sc84] E. V. Schepin, Soft mappings of manifolds, Russian Math. Surveys 39:5 (1984), $209-224$ (in Russian).

[Sh57] A. Shapiro, Obstructions to the embedding of a complex in a Euclidean space, I, The first obstruction, Ann. of Math. (2) 66 (1957), 256-269.

[Si69] K. Sieklucki, Realization of mappings, Fund. Math. 65 (1969), 325-343.

[Sk94] A. Skopenkov, A geometric proof of the Neuwirth theorem on thickenings of 2-polyhedra, Mat. Zametki 56:2 (1994), 94-98 (in Russsian); English transl.: Math. Notes, 58:5 (1995), 1244-1247.

[Sk95] A. Skopenkov, A description of continua basically embeddable in $\mathbb{R}^{2}$, Topol. Appl. 65 (1995), 29-48.

[Sk97] A. B. Skopenkov, On the deleted product criterion for embeddability of manifolds in $\mathbb{R}^{m}$, Comment. Math. Helv. 72 (1997), 543-555.

[Sk98] A. B. Skopenkov, On the deleted product criterion for embeddability in $\mathbb{R}^{m}$, Proc. Amer. Math. Soc. 126:8 (1998), 2467-2476.

[Sk00] A. Skopenkov, On the generalized Massey-Rolfsen invariant for link maps, Fund. Math. 165 (2000), 1-15.

[Sk02] A. Skopenkov, On the Haefliger-Hirsch-Wu invariants for embeddings and immersions, Comment. Math. Helv. 77 (2002), 78-124.

[Sk03] M. Skopenkov, Embedding products of graphs into Euclidean spaces, Fund. Math. 179 (2003), 191-198.

[Sk03'] M. Skopenkov, On approximability by embeddings of cycles in the plane, Topol. Appl. 134 (2003), $1-22$.

[Sk05] A. Skopenkov, A new invariant and parametric connected sum of embeddings, submitted to Fund. Math.; http://arxiv.org/abs/math/0509621.

[Sk05'] A. Skopenkov, On the Kuratowski graph planarity criterion, Mat. Prosveschenie 9 (2005), 116$128 ; 10$ (2006), 276-277.

[Sk05"] A. Skopenkov, Algebraic topology from geometrical point of view (in Russian), http:// www.mccme.ru/ ium/ s05/ el_al_top.html.

[Sk06] A. Skopenkov, Classification of smooth embeddings of 3-manifolds in the 6-space, submitted; http://arxiv.org/abs/math/0603429.

[Sk] A. Skopenkov, Classification of embeddings below the metastable range, preprint (2003).

[Sk'] M. Skopenkov, Explicit formulas for the groups of links and link maps, preprint.

[Sm42] P. A. Smith, Fixed points of periodic transformations, 350-373, Appendix B in: Algebraic Topology by S. Lefschetz (American Mathematical Society, New York, 1942).

[Sm59] S. Smale, The classification of immersions of spheres in Euclidean spaces, Ann. of Math. (2) 69 (1959), 327-344.

[Sp90] S. Spież, Imbeddings in $\mathbb{R}^{2 m}$ of $m$-dimensional compacta with $\operatorname{dim}(X \times X)<2 m$, Fund. Math. 134 (1990), 105-115.

[SS83] E. V. Schepin and M. A. Shtanko, A spectral criterion for embeddability of compacta in Euclidean space, Proc. Leningrad Int. Topol. Conf. (1983), Nauka, Leningrad, 135-142. (in Russian)

[SS92] J. Segal and S. Spież, Quasi embeddings and embeddings of polyhedra in $\mathbb{R}^{m}$, Topol. Appl. 45 (1992), 275-282.

[SSS98] J. Segal, A. Skopenkov and S. Spież, Embeddings of polyhedra in $\mathbb{R}^{m}$ and the deleted product obstruction, Topol. Appl. 85 (1998), 225-234.

[St63] J. Stallings, On topologically unknotted spheres, Ann. of Math. (2) 77 (1963), 490-503.

[St65] J. Stallings, Homology and central series of groups, J. of Algebra 2 (1965), 170-181.

[St89] Y. Sternfeld, Hilbert's 13th problem and dimension, Lect. Notes Math 1376 (1989), 1-49.

[ST91] S. Spież and H. Toruńczyk, Moving compacta in $\mathbb{R}^{m}$ apart, Topol. Appl. 41 (1991), $193-204$. 
[St] J. Stallings, The embedding of homotopy type into manifolds, mimeographed notes, Princeton Univ. (1965).

[Sz82] A. Szücs, The Gromov-Eliashberg proof of Haefliger's theorem, St. Sci. Math. Hung. 17 (1982), 303-318.

[Ta95] K. Taniyama, Homology classification of spatial embeddings of a graph, Topol. Appl. 65 (1995), $205-228$.

[Ti69] R. Tindell, Knotting tori in hyperplanes, in: Conf. on Topology of Manifolds, Prindle, Weber and Schmidt (1969), 147-153.

[To62] H. Toda, Composition Methods in the Homotopy Groups of Spheres, Princeton Univ. Press, Princeton, 1962.

[Va92] V. A. Vassiliev, Complements of discriminants of smooth maps: Topology and applications, Amer. Math. Soc., Providence, RI, 1992.

[Vr77] J. Vrabec, Knotting a k-connected closed $P L$ m-manifolds in $\mathbb{R}^{2 m-k}$, Trans. Amer. Math. Soc. 233 (1977), 137-165.

[Vr89] J. Vrabec, Deforming of a PL submanifold of a Euclidean space into a hyperplane, Trans. Amer. Math. Soc. 312:1 (1989), 155-178.

[Wa64] C. T. C. Wall, Differential topology, IV (theory of handle decompositions), Cambridge (1964), mimeographed notes.

[Wa65] C. T. C. Wall, All 3-manifolds imbed in 5-space, Bull. Amer. Math. Soc. 71 (1965), 490-503.

[Wa65'] C. T. C. Wall, Unknotting spheres in codimension two and tori in codimension one, Proc. Camb. Phil. Soc. 61 (1965), 659-664.

[Wa66] C. T. C. Wall, Classification problems in differential topology, IV, Thickenings, Topology 5 (1966), 73-94.

[Wa66'] C. T. C. Wall, Classification problems in differential topology, V, On certain 6-manifolds, Invent. Math. 1 (1966), 355-374.

[Wa70] C. T. C. Wall, Surgery on compact manifolds, Academic Press, London, 1970.

[We67] C. Weber, Plongements de polyèdres dans le domain metastable, Comment. Math. Helv. 42 (1967), 1-27.

[We68] C. Weber, Deux remarques sur les plongements d'un AR dans un éspace euclidien, Bull. Acad. Polon. Sci. Ser. Sci. Math. Astronom. Phys 16 (1968), 851-855.

[We] M. Weiss, Second and third layers in the calculus of embeddings, preprint.

[Wh35] H. Whitney, Differentiable manifolds in Euclidean space, Proc. Nat. Acad. Sci. USA 21:7 (1935), $462-464$.

[Wh44] H. Whitney, The self-intersections of a smooth n-manifolds in 2n-space, Ann. of Math (2) 45 (1944), 220-246.

[Wu58] W. T. Wu, On the realization of complexes in a Euclidean space, I, Sci Sinica 7 (1958), 251-297; II, Sci Sinica 7 (1958), 365-387; III, Sci Sinica 8 (1959), 133-150.

[Wu59] W. T. Wu, Science Record, N.S. 3 (1959), 342-351.

[Wu65] W. T. Wu, A Theory of Embedding, Immersion and Isotopy of Polytopes in an Euclidean Space, Science Press, Peking, 1965.

[Ya54] C. T. Yang, On theorems of Borsuk-Ulam, Kakutani-Yamabe-Yujobo and Dyson, I, Ann. of Math. 60 (1954), 262-282.

[Ze60] E. C. Zeeman, Unknotting spheres, Ann. of Math. (2) 72 (1960), 350-360.

[Ze62] E. C. Zeeman, Isotopies and knots in manifolds, In: Topology of 3-Manifolds, Prentice-Hall, Englewood Cliffs, Ed. M. K. Fort, N.J. (1962).

[Ze63] E. C. Zeeman, Unknotting combinatorial balls, Ann. of Math. 78 (1963), 501-526.

[Zh75] A. V. Zhubr, A classification of simply-connected spin 6-manifolds (in Russian), Izvestiya AN SSSR 39:4 (1975), 839-856.

[Zh89] A. V. Zhubr, Classification of simply-connected topological 6-manifolds, Lecture Notes in Math. 1346 (1989), 325-339.

[Zh94] L. Zhongmou, Every 3-manifold with boundary embeds in Triod $\times$ Triod $\times I$, Proc. AMS 122:2 (1994), 575-579.

Department of Differential Geometry, Faculty of Mechanics and Mathematics, Moscow State University, Moscow, Russia 119992, and Independent University of Moscow, B. Vlasyevskiy, 11, 119002, Moscow, Russia. E-MaIL: SKopenko@MCCME.RU 